University of Louisville

ThinkIR: The University of Louisville's Institutional Repository

$5-2020$

\title{
Sulforaphane protects against ethanol-induced apoptosis and teratogenesis through epigenetic modulation of anti-apoptotic genes.
}

Yihong Li

University of Louisville

Follow this and additional works at: https://ir.library.louisville.edu/etd

Part of the Other Pharmacy and Pharmaceutical Sciences Commons

\section{Recommended Citation}

$\mathrm{Li}$, Yihong, "Sulforaphane protects against ethanol-induced apoptosis and teratogenesis through epigenetic modulation of anti-apoptotic genes." (2020). Electronic Theses and Dissertations. Paper 3417. https://doi.org/10.18297/etd/3417

This Doctoral Dissertation is brought to you for free and open access by ThinkIR: The University of Louisville's Institutional Repository. It has been accepted for inclusion in Electronic Theses and Dissertations by an authorized administrator of ThinkIR: The University of Louisville's Institutional Repository. This title appears here courtesy of the author, who has retained all other copyrights. For more information, please contact thinkir@louisville.edu. 


\title{
SULFORAPHANE PROTECTS AGAINST ETHANOL-INDUCED APOPTOSIS AND TERATOGENESIS THROUGH EPIGENETIC MODULATION OF ANTI- APOPTOTIC GENES
}

\author{
By \\ Yihong Li \\ B.S. Qiqihar University, 2011 \\ M.S. Anhui University, 2014
A Dissertation
Submitted to the Faculty of the
School of Medicine of the University of Louisville
In partial Fulfillment of the Requirements
for the Degree of \\ Doctor of Philosophy in Pharmacology and Toxicology \\ Department of Pharmacology and Toxicology \\ University of Louisville \\ Louisville, KY
}

May 2020 



\section{SULFORAPHANE PROTECTS AGAINST ETHANOL-INDUCED APOPTOSIS AND TERATOGENESIS THROUGH EPIGENETIC MODULATION OF ANTI- APOPTOTIC GENES}

By

Yihong Li

B.S. Qiqihar University, 2011

M.S. Anhui University, 2014

Dissertation Approved on

April 3, 2020

By the following Dissertation Committee

Shao-yu Chen, Ph. D., (Mentor)

Walter H. Watson, Ph.D.

Lu Cai, Ph.D.

John P. Wise Sr, Ph.D.

Qunwei Zhang, Ph.D 


\section{DEDICATION}

This Dissertation is dedicated to my Parents

Jingfan Dong and Chunzhi Liu

And to my husband

Huadong Fan

For their endless love, patience and encouragement

In all my endeavors 


\section{ACKNOWLEDGEMENTS}

First and foremost, I would like to thank my mentor, Dr. Shao-yu Chen, for his continuous guidance and support of my Ph.D study and research, for his motivation, patience and immense knowledge, and his help in improving my abilities of critical thinking on the research project, as well as improving my writing skills. I would also like to thank all my committee members, including Dr. Lu Cai, Dr. Jone Pierce Wise. Sr, Dr. Walter Walson, Dr. Qunwei Zhang, for their scientific support, insightful comments, as well as great suggestions.

I am also grateful to Dr. David W. Hein and Dr. Lu Cai, for their efforts to build the Ph.D. program between Wenzhou Medical University and the University of Louisville, which offers me the opportunity to study here. I would also like to thank the present and former faculty and staff in our department for your support and help with my graduate life.

Many thanks to my labmates, past and present. I am grateful to Jie Liu for her help and lab management, Dr. Fuqiang Yuan for his help with hNCCs differentiation experiments, Dr. Ting Wu for her help with the animal study, Dr. Lanhai Lu for his comments on my research, and Dr. Huadong Fan for his tremendous help with various experiments. 
Thanks to Louisville, which is a lucky place for me. I met my husband here and we have a beautiful baby after two years of marriage. They are the most precious gift in my graduate journey.

Finally, I would like to thank my friends and family for their support throughout my years in graduate school. I would like to thank my parents, parents-in-law and my niece Qiqi for all of their loving support throughout my life. I would like to thank my husband, Huadong Fan, for always being there when I need him. All your love and support have been tremendous. Thank you. 


\begin{abstract}
SULFORAPHANE PROTECTS AGAINST ETHANOL-INDUCED APOPTOSIS AND TERATOGENESIS THROUGH EPIGENETIC MODULATION OF ANTIAPOPTOTIC GENES
\end{abstract}

Yihong Li

April 3, 2020

Background. Ethanol-induced excessive apoptosis in neural crest cells (NCCs), a multipotent progenitor cell population, is one of the major mechanisms underlying the pathogenesis of Fetal Alcohol Spectrum Disorders (FASD). However, the molecular mechanisms underlying FASD that results from maternal alcohol exposure during pregnancy are poorly understood. The overall goals of this study are to examine the mechanisms by which ethanol induces apoptosis and malformations in vitro and in vivo, and to develop a nutritional-based approach by using SFN and SFN-rich BSE to prevent FASD through epigenetic modulation. Results. This study demonstrates that ethanol exposure resulted in a significant increase in the DNMT activity and the expression of DNMT3a in human neural 
crest cells. SFN can significantly diminish ethanol-induced increases in DNMT activity and the expression of DNMT3a. We have also found that ethanol-induced up-regulation of DNMT3a and an increase in DNMT activity resulted in hypermethylation at the promoters of the selected anti-apoptotic genes and that SFN can diminish ethanol-induced hypermethylation at the promoters of the antiapoptotic genes by preventing ethanol-induced up-regulation of DNMT3a and increase in DNMT activity. In addition, the knockdown of DNMT3a or treatment with SFN significantly diminished ethanol-induced decreases in the mRNA and protein expression of NAIP and XIAP and prevented ethanol-induced apoptosis in human neural crest cells. The knockdown of DNMT3a also enhanced the effects of SFN on the mRNA and protein expression of NAIP and XIAP and the protective effects of SFN on ethanol-induced apoptosis. This study also shows that ethanol exposure can increase HDAC activity and the expression of HDAC2 in human neural crest cells. SFN treatment significantly diminished ethanol-induced increase in HDAC activity and the up-regulation of HDAC2. We have also found that ethanol-induced increase in HDAC activity and up-regulation of HDAC2 resulted in the reduction of $\mathrm{H} 3$ acetylation at the promoters of AKT1, BIRC6 and XIAP and that SFN diminished ethanol-induced reduction of $\mathrm{H} 3$ acetylation at the promoters of anti-apoptotic genes by inhibiting HDAC activity and reducing ethanol-induced up-regulation of HDAC2. In addition, SFN treatment or knockdown of HDAC2 significantly diminished ethanol-induced decreases in the mRNA and protein expression of AKT1, BIRC6 and XIAP and prevented ethanol-induced apoptosis in human neural crest cells. The knockdown of HDAC2 also enhanced the effects 
of SFN on the mRNA and protein expression of AKT1, BIRC6 and XIAP and the protection against ethanol-induced apoptosis. In addition, our studies have shown that ethanol exposure can inhibit EMT through the down-regulation of Snail1 by decreasing $\mathrm{H} 3 \mathrm{~K} 4 \mathrm{me} 3$ enrichment at the promoter regions of Snail1 and increase apoptosis in neural crest cells. SFN treatment can reverse the ethanol-induced reduction of the H3K4me3 enrichment at the promoter regions of Snail1, restore the expression of Snail1 and EMT in neural crest cells exposed to ethanol and diminished ethanol-induced apoptosis. These findings demonstrate that the disruption of EMT contributes to ethanol-induced apoptosis in neural crest cells and that SFN can prevent ethanol-induced apoptosis by restoring EMT through epigenetically regulating the expression of EMT-related genes, suggesting that elucidation of Snail1's role in EMT and ethanol-induced apoptosis in neural crest cells may provide critical insight into the pathogenesis of FASD. Moreover, this study has demonstrated that SFN-rich BSE can attenuate ethanol-induced teratogenesis through epigenetically up-regulating the anti-apoptotic genes. Conclusions. The findings from work presented in this dissertation provide critical insight into the pathogenesis of FASD. In addition, the potency of SFN in preventing ethanol-induced apoptosis illustrates the potential of a practical and promising therapeutic strategy for FASD. 
TABLE OF CONTENTS

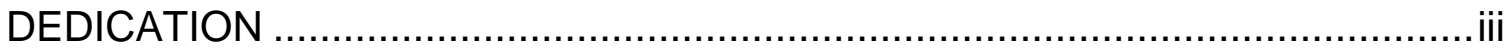

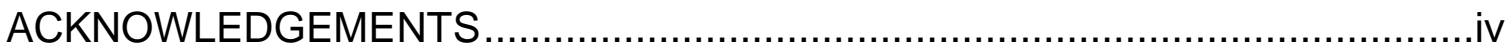

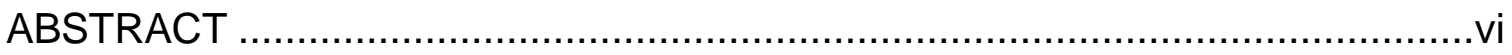

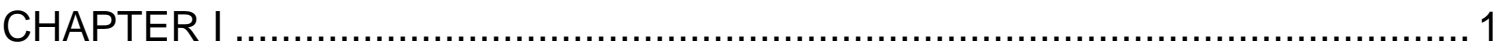

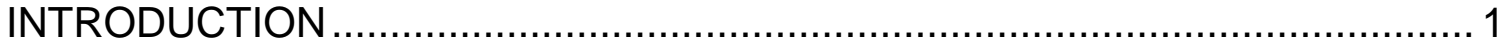

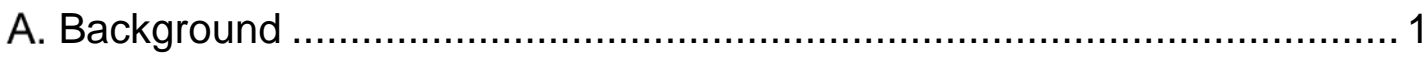

1. FASD and ethanol-induced apoptosis in NCCs ................................... 1

2. Epigenetic regulation and FASD................................................... 3

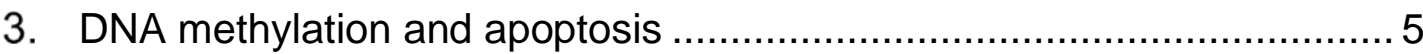

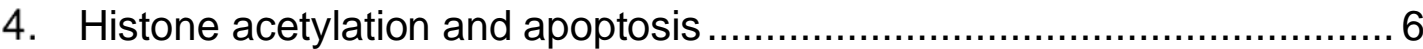

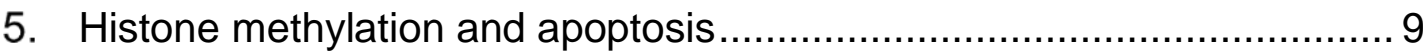

6. Epigenetic cross-talk between DNA methylation and histone

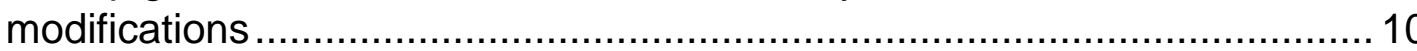

7. Epithelial-mesenchymal transition (EMT) and neural crest cells

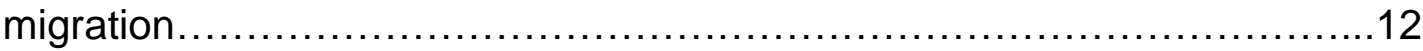

8. Sulforaphane is an inhibitor for both DNMTs and HDACs .................... 13

9. Broccoli sprout extract (BSE) ............................................................. 14

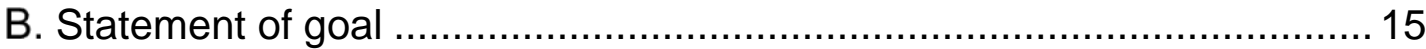

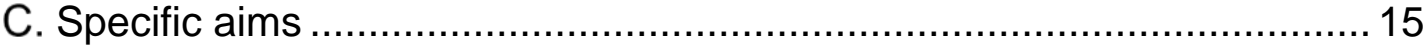

1. Aim1: To identify the anti-apoptotic genes that are epigenetically silenced by ethanol and de-repressed by SFN in ethanol-induced hNCCs.. 15

2. Aim2: To further explore the mechanism by which SFN epigenetically modulates the anti-apoptotic genes and apoptosis................................... 16

3. Aim 3: To explore the novel therapeutic approach for the intervention of ethanol-induced teratogenesis through maternal dietary administration of SFN-rich broccoli sprout....................................................................... 16

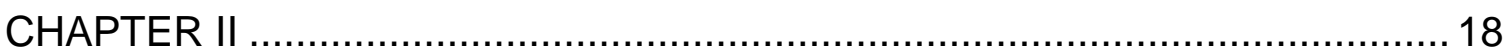

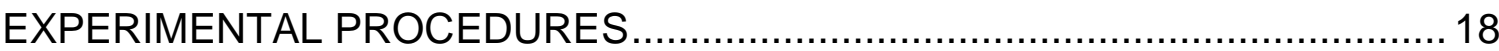




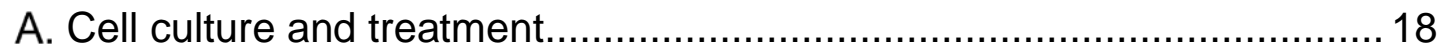

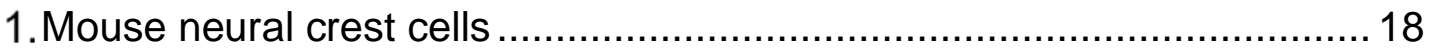

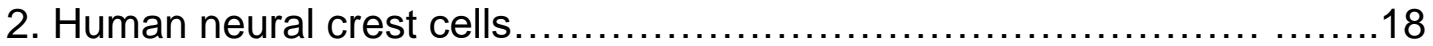

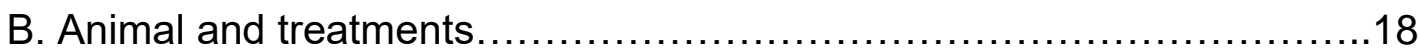

1. Mice maintenance, time mating and BSE treatment............................ 19

2. Fish maintenance, mating and treatment ......................................... 21

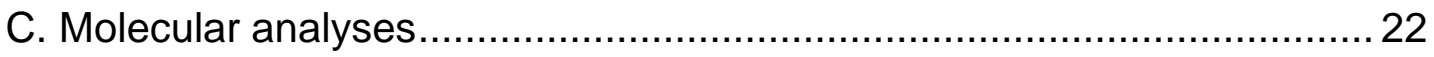

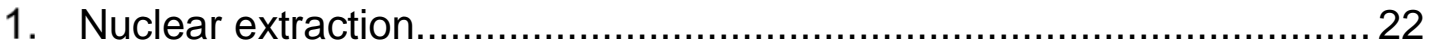

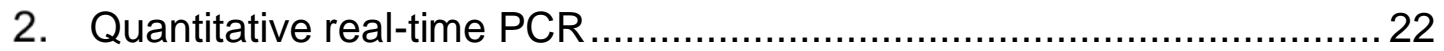

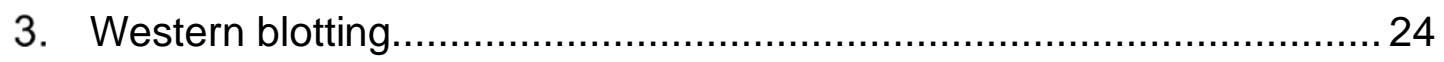

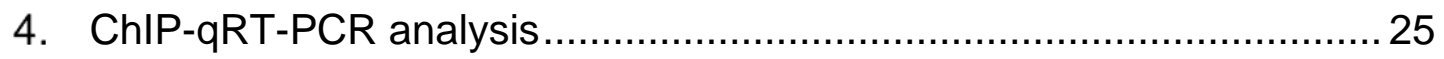

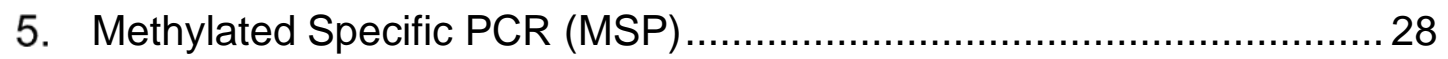

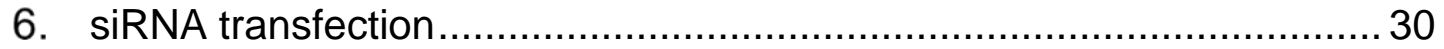

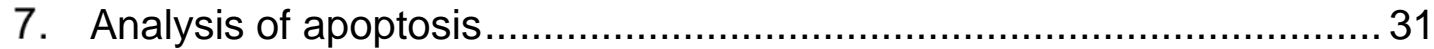

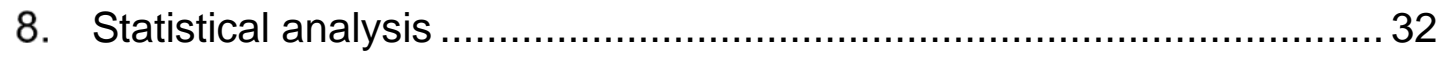

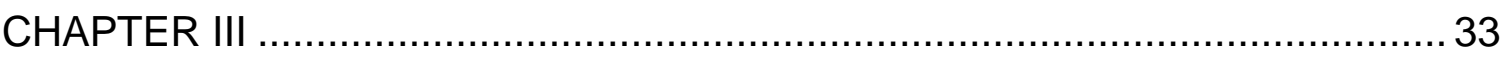

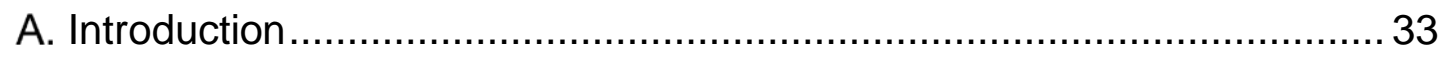

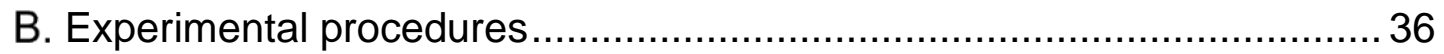

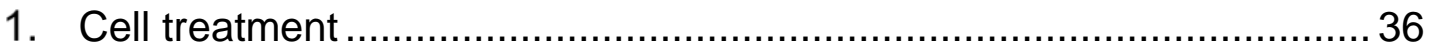

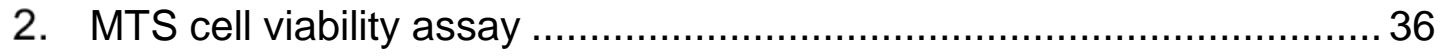

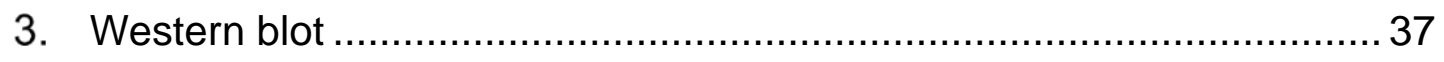

4. Expression profiling of apoptosis-related genes using human apoptosis

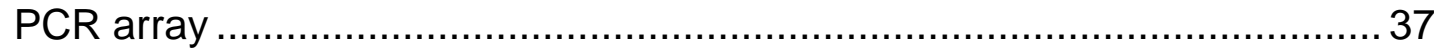

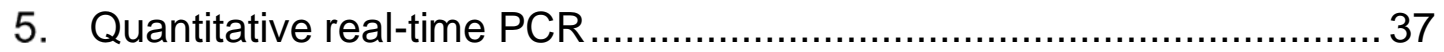

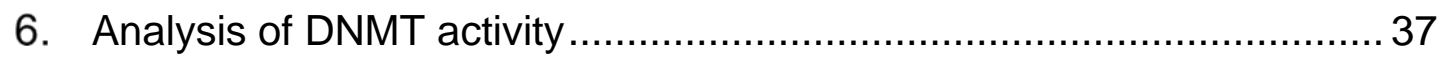

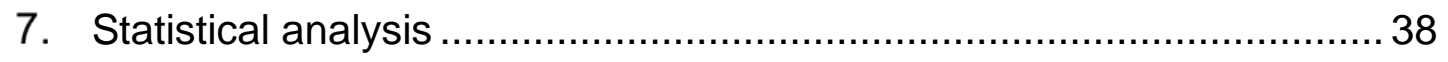

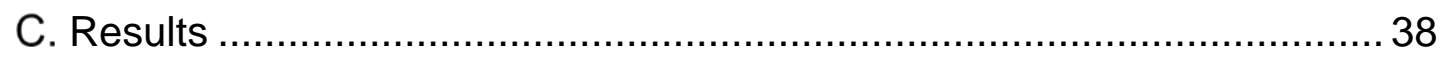

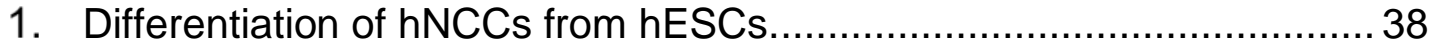

2. Ethanol treatment significantly increased apoptosis in hNCCs............. 40

3. SFN treatment prevented ethanol-induced apoptosis in hNCCs. .......... 42

4. Identification of anti-apoptotic genes that are repressed by ethanol and that their repression by ethanol can be prevented by SFN in hNCCs......... 44 
5. SFN diminished ethanol-induced increase in DNMT activity and upregulation of DNMT3a in hNCCs.

6. SFN diminished the ethanol-induced hypermethylation at the promoters of the selected anti-apoptotic genes in hNCCs.

7. Knockdown of DNMT3a and SFN treatment significantly diminished ethanol-induced down-regulation of NAIP and XIAP in hNCCs

8. Knockdown of DNMT3a and SFN treatment prevented ethanol-induced

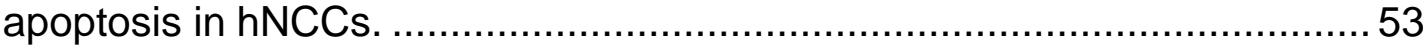

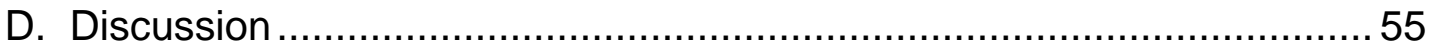

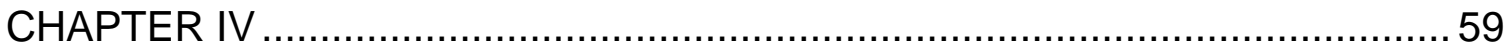

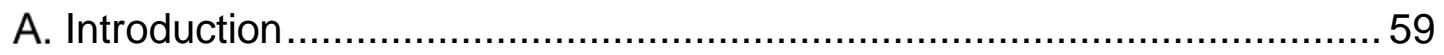

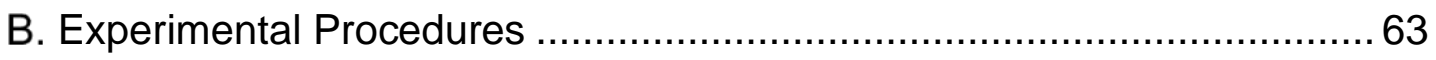

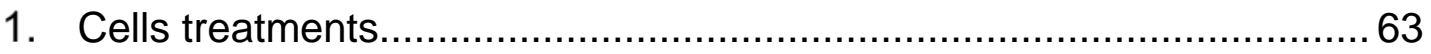

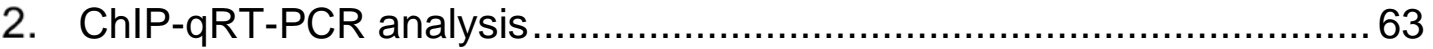

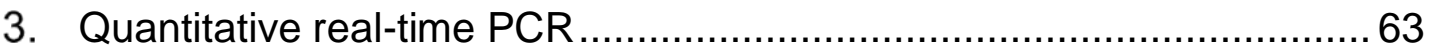

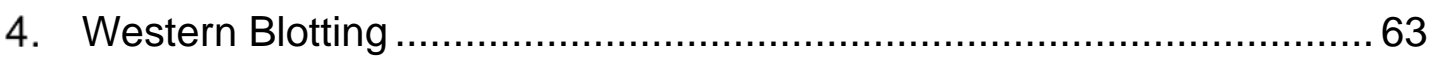

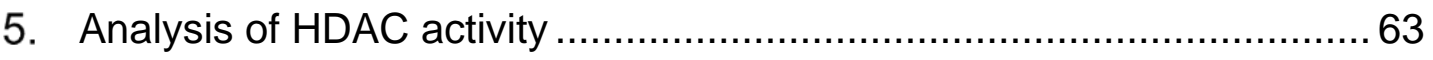

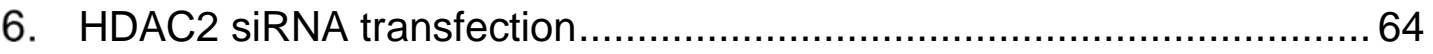

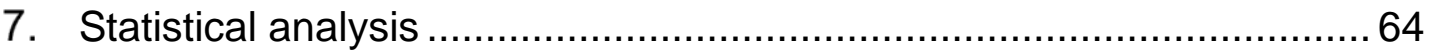

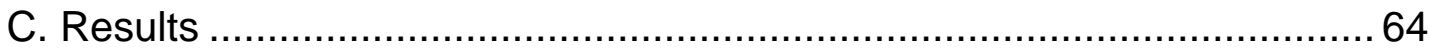

1. SFN diminished the ethanol-induced increase HDAC activity and the up-

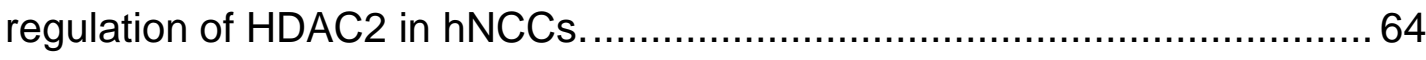

2. SFN diminished the ethanol-induced reduction of $\mathrm{H} 3$ acetylation at the promoters of the selected anti-apoptotic genes in hNCCs. .........................67

3. SFN treatment diminished ethanol-induced down-regulation of AKT1,

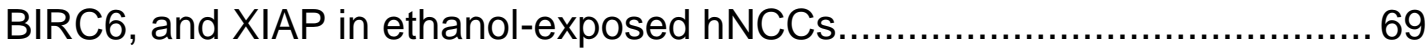

4. Knockdown of HDAC2 prevented ethanol-induced down-regulation of AKT1, XIAP and BIRC6 in hNCCs........................................................ 71

5. The knockdown of HDAC2 and SFN treatment prevented ethanolinduced apoptosis in hNCCs.................................................................... 73

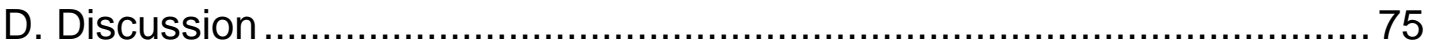

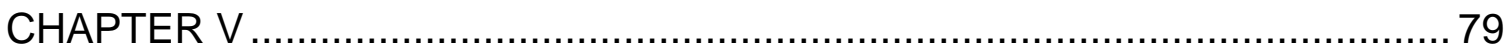

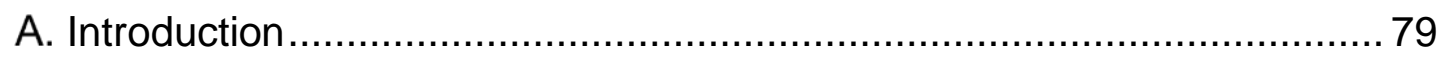

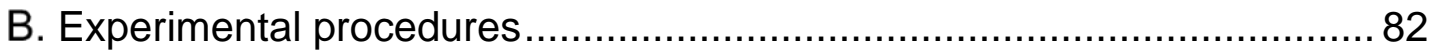

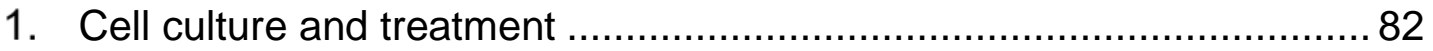




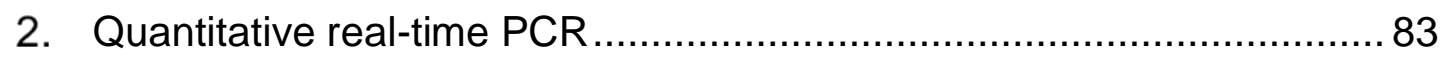

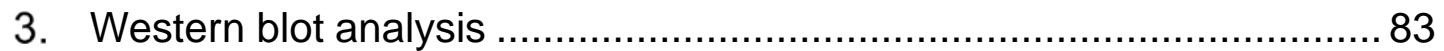

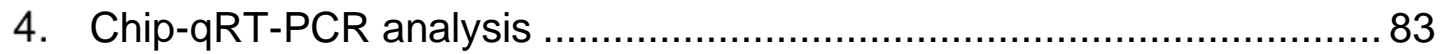

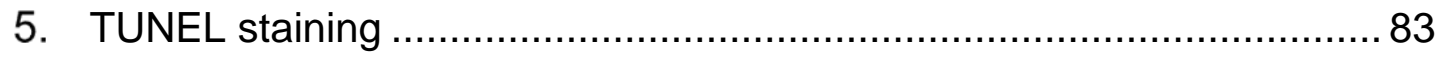

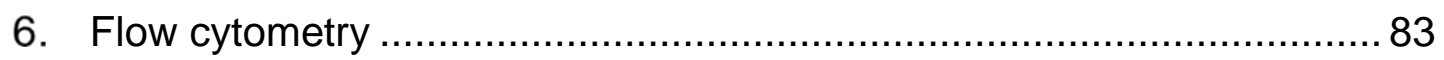

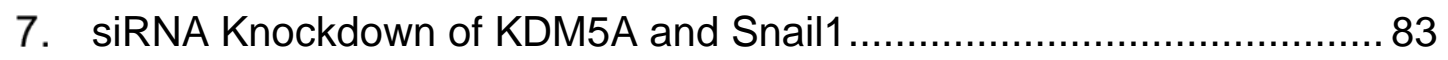

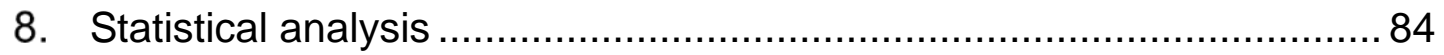

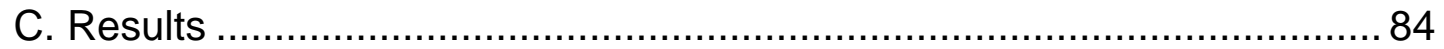

1. Ethanol exposure induced apoptosis in NCCs .................................... 84

2. SFN diminished ethanol-induced inhibition of EMT in NCCs ................ 86

3. SFN diminished the ethanol-induced increases in the mRNA and protein

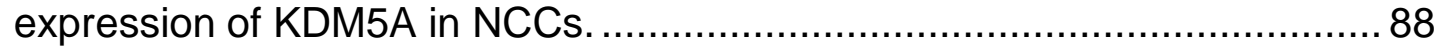

4. SFN treatment diminished the ethanol-induced reduction in the H3K4me3 enrichment at the promoter regions of the Snail1 gene in NCCs. 90 5. SFN treatment restored the expression of Snail1 in ethanol-exposed

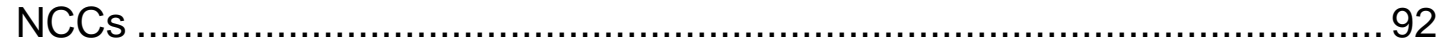

6. Treatment with SFN significantly decreased the up-regulation of Snail1 target gene E-cadherin in NCCs exposed to ethanol

7. SFN treatment significantly diminished ethanol-induced apoptosis in NCCs through up-regulation of Snail1

8. The knockdown of KDM5A significantly diminished the ethanol-induced inhibition of $\mathrm{H} 3 \mathrm{~K} 4 \mathrm{me} 3$ expression and reduction of $\mathrm{H} 3 \mathrm{~K} 4 \mathrm{me} 3$ enrichment at Snail1 promoters.

9. Down-regulation of KDM5A diminished ethanol-induced inhibition of EMT in NCCs. 100

10. The knockdown of KDM5A significantly decreased ethanol-induced apoptosis in NCCs and zebrafish embryos. 102

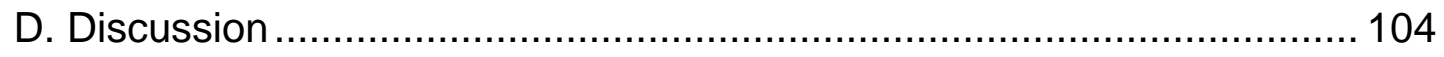

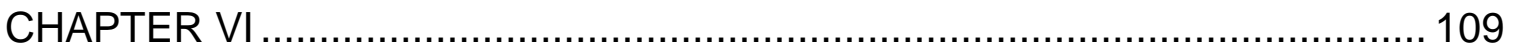

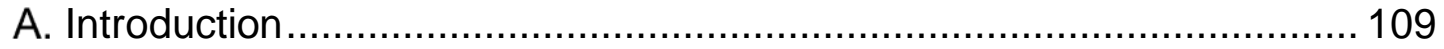

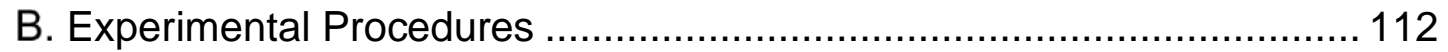

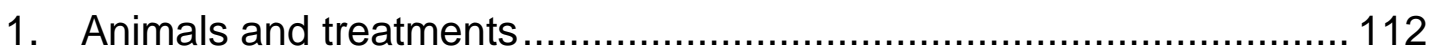

2. Methylated specific PCR (MSP) …............................................. 113

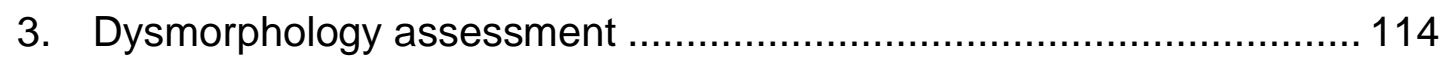

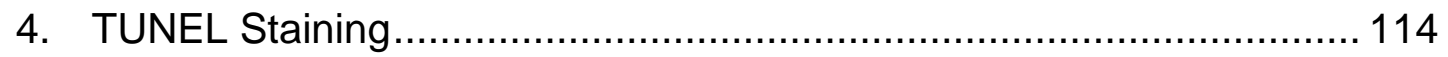




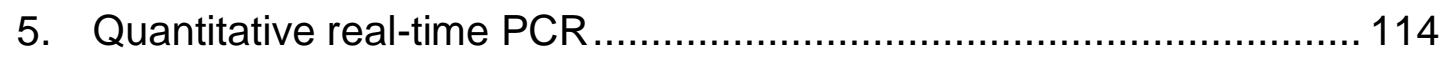

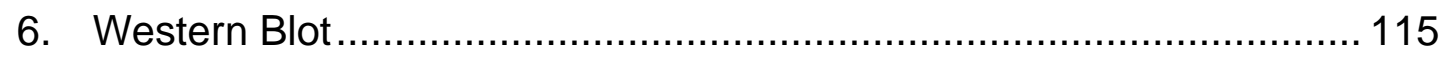

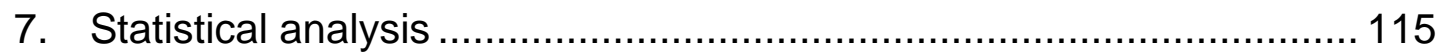

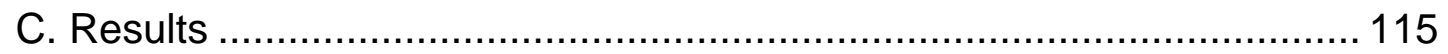

1. Maternal dietary BSE administration significantly reduced ethanolinduced hypermethylation at the promoters of the selected anti-apoptotic genes in mouse embryos.

2. Maternal dietary BSE reduced the ethanol-induced down-regulation of the selected anti-apoptotic genes in mouse embryos

3. Maternal dietary BSE protected against ethanol-induced apoptosis in mouse embryos

4. Maternal dietary BSE confers in vivo protection against ethanol-induced teratogenesis in mouse embryos.

5. Maternal dietary administration of BSE prevented ethanol-induced ocular

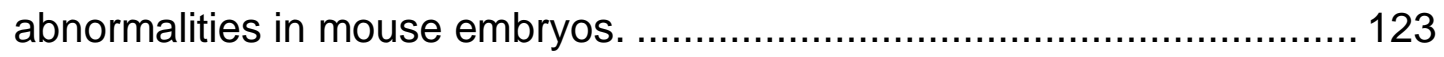

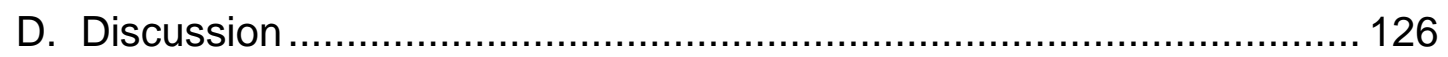

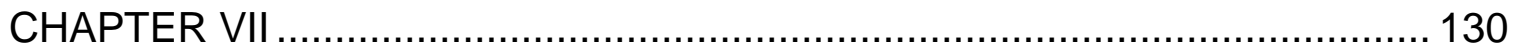

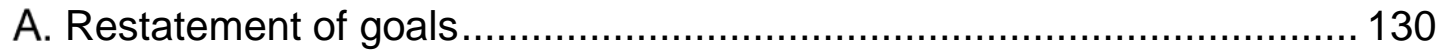

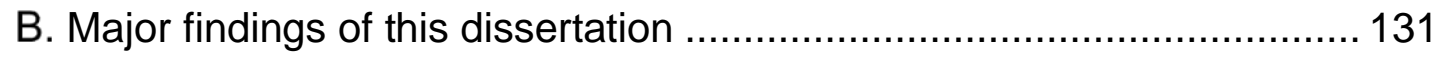

1. SFN protects against ethanol-induced apoptosis in hNCCs through diminishing ethanol-induced hypermethylation at the promoters of the antiapoptotic genes.

2. SFN prevents hNCCs from ethanol-induced apoptosis by diminishing ethanol-induced reduction of histone acetylation at the promoters of the antiapoptotic genes.

3. SFN protects against ethanol-induced apoptosis in NCCs and zebrafish embryos through modulating histone methylation and the expression of Snail 1 and restoring EMT.

4. Maternal dietary administration of SFN-rich BSE diminished ethanolinduced apoptosis and teratogenesis in mouse embryos by epigenetically modulating anti-apoptotic genes. ...................................................... 133

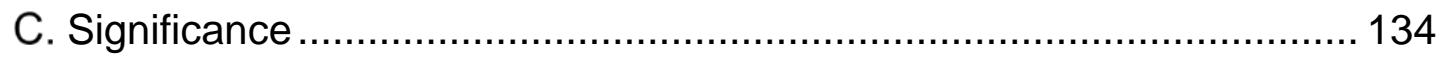

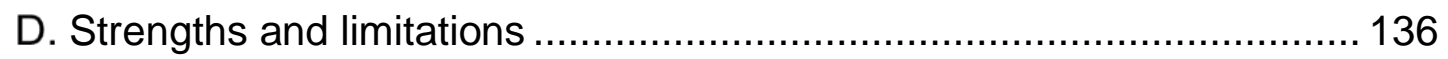

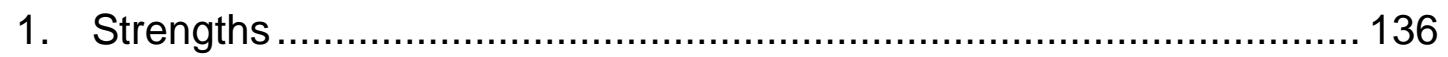

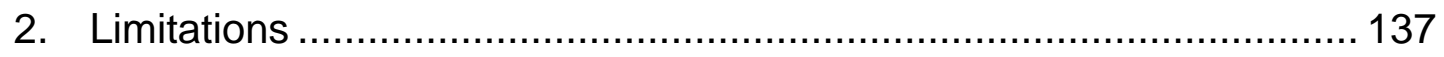

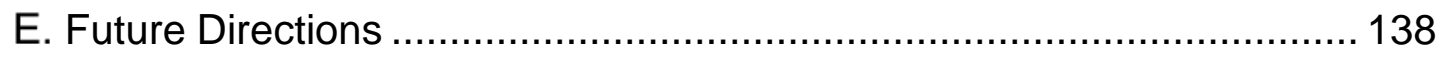


1. Elucidation of the molecular mechanisms underlying ethanol-induced increase in the activity of DNMTs and HDACs and up-regulation of DNMT3a, HDAC2 and KDM5A. 139

2. Determination of the synergistic pro-apoptotic effects of epigenetic modulators, DNMTs, HDACs and KDM in ethanol-exposed hNCCs.......... 139

F. Summary and conclusions ............................................................... 140

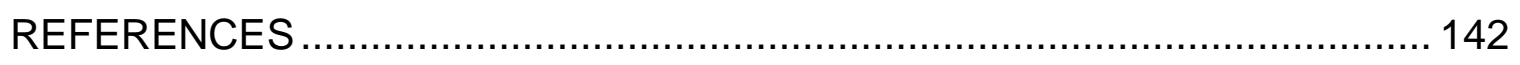

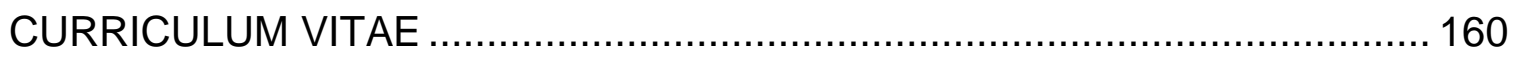




\section{LIST OF FIGURES}

Figure 3. 1. Differentiation of hNCCs from hESCs. Immunocytochemistry analysis shows that hNCCs are positive for the NCC marker HNK1.

Figure 3. 2. Ethanol exposure significantly reduced cell viability and increased apoptosis in hNCCs.

Figure 3. 3. SFN prevented the ethanol-induced reduction of cell viability and apoptosis in hNCCs.

Figure 3. 4. Identification of the anti-apoptotic genes that are repressed by ethanol and de-repressed by SFN.

Figure 3. 5. Co-treatment with SFN and ethanol reduced DNMT activity and DNMT3a expression in ethanol exposed hNCCs.

Figure 3. 6. SFN significantly diminished the ethanol-induced hypermethylation at the promoters of $\mathrm{Bcl} 2, \mathrm{XIAP}, \mathrm{BIRC} 5, \mathrm{BIRC} 6$ and NAIP in hNCCs.

Figure 3. 7. Knockdown of DNMT3a and SFN treatment significantly diminished ethanol-induced down-regulation of NAIP and XIAP in hNCCs.

Figure 3. 8. Knockdown of DNMT3a and SFN treatment prevented ethanolinduced apoptosis in hNCCs

Figure 4. 1. SFN treatment significantly decreased HDAC activity and HDAC2 expression in ethanol-exposed hNCCs.

Figure 4. 2. SFN increased acetyl-histone $\mathrm{H} 3$ protein levels and histone $\mathrm{H} 3$ acetylation at the promoters of AKT1, BIRC5, and XIAP in ethanol-exposed hNCCs..

Figure 4. 3. SFN treatment significantly increased the mRNA expression of AKT1, BIRC6 and XIAP in ethanol-exposed hNCCs................................ 70

Figure 4. 4. Knockdown of HDAC2 prevented ethanol-induced down-regulation of AKT1, XIAP and BIRC6 in hNCCs. 72

Figure 4. 5. The knockdown of HDAC2 by siRNA or SFN treatment prevented ethanol-induced apoptosis in hNCCs. 
Figure 5. 1. Ethanol exposure induced apoptosis in NCCs.. ........................... 85

Figure 5. 2. SFN diminished ethanol-induced EMT inhibition in NCCs.............. 87

Figure 5. 3. SFN diminished ethanol-induced increases in the mRNA and protein expression of KDM5A in NCCs.

Figure 5. 4. SFN diminished the ethanol-induced reduction of the levels of

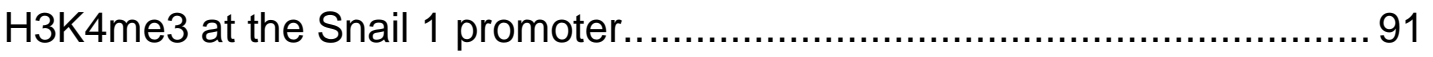

Figure 5. 5. SFN restored Snail1 expression in ethanol-exposed NCCs. ........... 93

Figure 5. 6. SFN diminished the expression of Snail1 target gene E-cadherin in

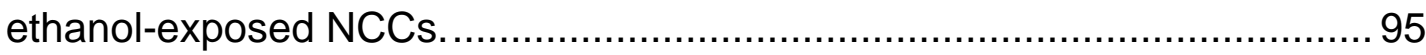

Figure 5. 7. SFN diminished ethanol-induced apoptosis in NCCs through the upregulation of Snail1. 97

Figure 5. 8. Down-regulation of KDM5A significantly diminished ethanol-induced inhibition of $\mathrm{H} 3 \mathrm{~K} 4 \mathrm{me} 3$ expression and reduction of $\mathrm{H} 3 \mathrm{~K} 4 \mathrm{me} 3$ enrichment at the Snail1 promoter regions in NCCs.

Figure 5. 9. Down-regulation of KDM5A diminished ethanol-induced inhibition of EMT in NCCs.

Figure 5. 10. The knockdown of KDM5A significantly decreased -induced apoptosis in NCCs and zebrafish embryos..

Figure 6. 1. Maternal dietary BSE significantly diminished ethanol-induced hypermethylation at the promoters of AKT1, Bcl2 and NAIP in mouse embryos..

Figure 6. 2. Maternal dietary BSE prevented ethanol-induced down-regulation of anti-apoptotic genes in mouse embryos.

Figure 6. 3. Maternal dietary BSE protects against ethanol-induced apoptosis in mouse embryos.

Figure 6. 4. BSE administration confers in vivo protection against ethanolinduced teratogenesis.

Figure 6. 5. Maternal dietary administration of BSE prevented ethanol-induced ocular abnormalities in mouse embryos 


\section{CHAPTER I}

\section{INTRODUCTION}

\section{A. Background}

\section{FASD and ethanol-induced apoptosis in NCCs}

Fetal alcohol spectrum disorder (FASD) is one of the most common birth defects caused by maternal drinking alcohol during pregnancy. The recent metaanalysis estimated that the worldwide prevalence of FASD is approximately 7.7 per 1000 population ${ }^{1}$. The World Health Organization (WHO) European region had the highest prevalence of FASD, which is 19.8 per 1000 population ${ }^{1}$. In the United States, the active case ascertainment studies estimated that 1.1 to $4.8 \%$ of schoolaged children might have FASD². FASD is one of the major public health problems in this country and in the world.

FASD is characterized by craniofacial abnormalities, including narrow forehead, short palpebral fissures, elongated upper lip, upturned nose and deficient philtrum. In addition of the craniofacial dysmorphology, the long-term consequences of prenatal alcohol exposure include mental retardation, growth retardation, cognitive deficits and social problems. A number of animal models of prenatal alcohol exposure have been used to elucidate the mechanisms 
underlying the teratogenic effect of alcohol ${ }^{3-7}$. A number of studies have demonstrated that the teratogenesis caused by ethanol is, at least in part, mediated by excessive cell death in selected cell populations ${ }^{8}$. Among these, the neural crest cells (NCCs) are one of the most vulnerable cell populations to ethanol stimuli during the embryo development ${ }^{9}$. NCCs is a cell population that arises at the border of the non-neural ectoderm and the neural plate at the end of gastrulation. This border, also known as neural fold, flanks the neural plate bilaterally. During neurulation, the neural plate closes and brings together the neural folds at the dorsal midline. Subsequently, NCCs go through the epithelialto-mesenchymal transition (EMT), allowing them to delaminate from the neuroepithelium and to migrate ventrally and differentiate into various cell types ${ }^{10}$. In the cranial region, the neural crest gives rise to the majority of the skull and facial skeleton, teeth, cartilage, tendons, smooth muscles, dermis and melanocytes as well as neurons and glia of the cranial ganglia ${ }^{11-13}$. Studies by Kotch and Sulik, as well as Dunty et al. have shown that ethanol exposure during gastrulation and/ or neurulation resulted in massive cell apoptosis in hindbrain neuroepithelium as well as other cell populations including $\mathrm{NCCs}^{14}$. The study by Rovasio has also indicated that ethanol exposure could induce morphological and dynamic changes in NCCs in vivo and in vitro ${ }^{15}$. In addition, study also suggested that ethanol may directly interfere with the earliest neural crest formation by downregulation of the inducers of neural crest, which subsequently causes apoptosis in the neural crest ${ }^{16}$. The previous studies from our group have also demonstrated that ethanol 
significantly increased apoptosis in NCCs ${ }^{17-19}$, and that ethanol-induced apoptosis in NCCs contributes significantly to the ethanol-induced malformation.

\section{Epigenetic regulation and FASD}

Epigenetics can be defined as heritable changes in gene expression without alteration in the DNA sequences. The epigenetic modifications generally include DNA methylation and histone modification, of which histone can be modified by methylation and acetylation. Both DNA methylation and histone modification can alter the accessibility of double strands of DNA to the molecular transcriptional machinery. Epigenetic regulation can be interfered with by ethanol, leading to aberrant gene expression and subsequently contributing to the abnormal fetal development ${ }^{20,21}$.

Using animal models of FASD, substantial studies have reported that the epigenetic alternations, including DNA methylation ${ }^{22-24}$ and histone modification ${ }^{25-}$ ${ }^{29}$, are involved in the detrimental effects of prenatal alcohol exposure and contribute to the abnormalities associated with FASD. A study by Garro has shown that epigenetic modifications may occur after ethanol exposure during embryonic development through hypermethylation of fetal DNA, which may contribute to abnormalities in $\mathrm{FASD}^{30}$. Liu et al. found that extensive methylation occurred in the developmental genes in alcohol-exposed mouse embryos ${ }^{20}$. Study has also shown that exposure to alcohol increased the probability of transcriptional silencing of the $A^{v y}$ gene in early fetal development ${ }^{31}$. In addition, it was reported that prenatal alcohol exposure resulted in alterations in DNA methylation in mice and consequently led to craniofacial and growth defects that are paralleled to FASD in 
humans. Zhou and colleagues have also demonstrated that DNA methylation in the developing nervous system were disturbed by alcohol, leading to hypermethylation and the repression of the genes, particularly in the genes involved in neural tube development ${ }^{32,33}$. Moreover, the enhanced methylation was found in imprinted genes, which are essential for cell cycle, growth, apoptosis, cancer and in a significant number of genes essential for olfaction ${ }^{20}$. These results indicated that the ethanol-induced alterations in DNA methylation contribute to the pathogenesis of FASD.

In addition to DNA methylation, histone modifications also play an important role in ethanol-induced apoptosis and the pathogenesis of FASD. The earliest study on the effects of ethanol on histone modifications was conducted on liver cells. This study has demonstrated that H3K4ac was increased in a time and dosedependent manner in liver cells exposed to ethanol ${ }^{34}$. It was also reported that acute alcohol exposure in mice resulted in a strong increase in H3K9me2 and H3K9ac levels at GD7 and reduced H3K27me3 levels at GD17 and that these histone modifications correlated with the craniofacial development and the CNS defects. In the offspring of the alcohol-exposed mice, the H3K4me2 and H3K4me3 were reduced significantly while both acetylated H3K9 and H3K9me2 were increased ${ }^{35}$. Consistent with acetylated or methylated histone $\mathrm{H} 3$ and $\mathrm{H} 4$, the transcriptional activity of enzymes responsible for gene expression were also changed ${ }^{36}$. Those epigenetic changes can persist long after the transient ethanol signal has disappeared, highlighting the particular importance of this phenomenon for FASD. 


\section{DNA methylation and apoptosis}

During embryonic and fetal development, the epigenetic markers (including DNA methylation and histone modification) are well established, and its status at some genomic loci is labile and changes in response to environmental exposure, including diet and alcohol ${ }^{37}$. DNA methylation occurs on the cytosine residue of CpG dinucleotides and is performed by DNA methyltransferases (DNMTs) through the transfer of 5-methylcytosine from the methyl donor S-adenosylmethionine (SAM) to the CpG. Three DNMTs (DNMT1, DNMT3a, DNMT3b) are required for the establishment and maintenance of DNA methylation patterns. DNMT1 is responsible for the maintenance of established patterns of DNA methylation, while

DNMT3a and DNMT3b mediate the establishment of new or de novo DNA methylation patterns and play essential roles during embryonic development ${ }^{38}$. In mammal, DNA methylation is globally distributed in CpG islands throughout the entire genome, which are areas rich in CpG dinucleotides and typically located near the promoter region of genes. Alteration of the DNMT level may trigger hypermethylation or hypo-methylation of gene promoters, which could consequently result in inhibiting or activating gene expression, respectively. Evidence from literature indicates that the DNA methylation-mediated regulation of gene expression is involved in apoptosis, which is an important mechanism through which tumor cells avoid apoptosis ${ }^{39,40}$. These changes can occur either in the form of hypomethylation to reactivate anti-apoptotic genes or hypermethylation to repress pro-apoptotic genes ${ }^{40}$. A study by Pompeia et al. showed that aberrant methylation by DNMT inhibitor zebularine promoted the expression of some pro- 
apoptotic genes (BAD, BAK, BIK and BAX), contributing to growth and survival of multiple myeloma cells ${ }^{41}$. The other studies indicated that hypermethylation of the pro-apoptotic gene Fas in T-cell lymphoma or in colon carcinoma cell lines correlated to a decrease in Fas expression, thus conferring resistance to apoptosis ${ }^{42-44}$, whereas DNMT inhibitor 5-aza-2'-deoxycytidine (5-azadC) can reverse this effect. Similarly, a study that analyzed the methylation status of proapoptotic gene BNIP3 indicated that the methylation level correlated inversely with gene expression and also with the reduction of apoptosis ${ }^{45}$. In addition, it has been shown that the loss of methylation in the inhibitor apoptosis protein (IAP) promoters might be related to apoptosis resistance ${ }^{39}$. Strikingly, Survivin, which is normally expressed during embryonic development, has been found to be hypomethylated by DNMT inhibitor and highly overexpressed in different cancers including ovarian, HCC (Hepato cellular carcinoma) and glioma, conferring resistance to apoptosis ${ }^{39}$, 46. A positive correlation between DNMT1 levels and Survivin methylation status suggested that DNMT1 is required for Survivin regulation ${ }^{47}$. These studies demonstrate that alterations of methylation at the promoters of apoptotic genes are associated with apoptosis.

\section{Histone acetylation and apoptosis}

Histone modification is one of the major epigenetic modifications. Histone modifications play a crucial role in many cellular processes during embryonic development, including cell proliferation, cell survival and cellular differentiation ${ }^{48}$. In eukaryotic cells, DNA forms a complex structure with proteins called chromatin. In chromatin, the fundamental repeat unit is the chromosome, consisting of one 
octamer comprised of four core histone proteins $(\mathrm{H} 2 \mathrm{~A}, \mathrm{H} 2 \mathrm{~B}, \mathrm{H} 3$, and $\mathrm{H} 4)$ around which 147 base pairs of DNA are wrapped ${ }^{48,} 49$. Chromatin modifications also shape the epigenome and regulate gene expression in both normal and cancer cells ${ }^{50}$. Each of the core histones has an $\mathrm{N}$-terminal "tail" that can be subject to several covalent modifications associated with euchromatic (open) and heterochromatic (closed) chromatin states. These covalent modifications are established by "writers", such as histone lysine methyltransferase (KMTs), histone acetyltransferases (HATs). These modifications can be removed by "erasers", such as histone demethylases (KDMs), histone deacetylases (HDACs) ${ }^{51}$. HATs and HDACs are associated with histone acetylation and deacetylation, respectively. There are 18 HDACs in humans, and they are distributed into 4 classes: Class I HDACs include HDACs 1, 2, 3 and 8, which are located in the nucleus. Class I HDACs were reported to regulate neurogenesis, proliferation, differentiation, and embryonic development ${ }^{52-54}$. Class II HDACs include HDAC 4, 5, 9, and 10 (Class Ila) as well as HDACs 6 and 10 (Class Ilb), which are localized both in the nucleus and the cytoplasm. Class III HDACs comprise SIRTs 1 to 7 , and class IV is formed by HDAC11 only ${ }^{55}$. Both HATs and HDACs regulate the dynamic acetylation balance of histone and nonhistone proteins and play an important role in cell proliferation, apoptosis, differentiation, cancer treatment, neuroprotection, and anti-inflammatory effects ${ }^{56-58}$. The histone deacetylase inhibitor (HDACi) can interfere with the deacetylase function of HDACs, improve the acetylation level of histone, and regulate gene expression. Histone acetylation is associated with activation of transcription. Active genes usually carry high levels 
of lysine acetylation on histone $\mathrm{H} 3(\mathrm{H} 3 \mathrm{ac})$ and h4 (H4ac). Moreover, histone $\mathrm{H} 3$ lysine acetylation at position 9 (H3K9Ac) and 14 (H3K14Ac) is associated with open chromatin at the gene promoter region and activate gene expression. Substantial studies have shown that the HDACis are emerging as a prominent class of therapeutic agents for treatment of a variety of cancers, such as pancreatic, ovarian, breast, colon, prostate, and thyroid cancer ${ }^{59-63}$. Other studies have also demonstrated that HDACi has important neuroprotective effects in the treatment of diseases of nervous system ${ }^{64-67}$. HDACi are known to inhibit apoptosis, increase cell survival and enhance anti-inflammatory response ${ }^{64,65,68,69}$. The study by Dr. Chuang group showed that HDACi Trichostatin A (TSA) can regulate the expression of apoptosis-related genes Bcl2 and apaf-1 and improve neurological performance in the rat permanent middle cerebral artery occlusion (pMCAO) model of stoke ${ }^{65}$. Valproic acid (VPA), another HDACi, can also attenuate retinal neuron apoptosis by inhibiting the activation of caspase 3 and the apoptotic protease activating factor-1 (apaf-1), and the release of cytochrome $\mathrm{C}^{68}$. It has also been demonstrated that HDACi increased the acetylation of histone $\mathrm{H}$, activated the transcription of downstream genes Akt, Erk, CREB and HSP70, and thus upregulated the levels of anti-apoptotic proteins Bcl-2 and Bcl-xL, and eventually led to the downregulation of caspase- $3^{65,70}$. In addition, a study from Dr. Zhang shown that VPA protects against neuronal apoptosis by activation of BNDF-TrkB signaling at the transcriptional levels and that VPA treatment resulted in the hyperacetylation of histone H3K14, attenuated histone H3K9 hypermethylation in the BNDF promoter, and promoted transcriptional activity ${ }^{71}$. A study from this 
group has also demonstrated that VPA upregulated the expression of the antiapoptotic gene Hsp70 by enhancing the acetylation of histone $\mathrm{H} 3$ at $\mathrm{Hsp} 70$ promoter ${ }^{68}$. Our recent study has shown sulforaphane (SFN), a well-known HDACi, restored the expression of the anti-apoptotic gene $\mathrm{Bcl}-2$ and attenuated ethanolinduced apoptosis by enhancing histone $\mathrm{H} 3$ acetylation at the $\mathrm{Bcl}-2$ promoter in $\operatorname{NCCs}^{18}$.

\section{Histone methylation and apoptosis}

The other dominant histone modification is the methylation of lysine and arginine. The regulation of gene expression by histone methylation is correlated with specific histone methylation of lysine and arginine amino acids of histone Nterminal tails. Histone methylation is well characterized at lysine residues 4, 9, 27, and 36 of histone $\mathrm{H} 3$ and at lysine 20 of histone $\mathrm{H} 4$. The effects of histone modifications on gene expression depend on the position of lysine residues that are methylated. For example, active genes are marked at their transcript start site (TSS) by H3K4me3, which facilitates transcriptional initiation ${ }^{50}$. Moreover, high levels of lysine trimethylation of $\mathrm{H} 3$ lysine $79(\mathrm{H} 3 \mathrm{~K} 79 \mathrm{me} 3)$ and trimethylation of $\mathrm{H} 3$ lysine 36 (H3K36me3) at the promoter regions of genes also promotes gene expression $^{72}$. On the other hand, the histone $\mathrm{H} 3$ lysine 27 trimethylation (H3K27me3) and H3K9 methylation (H3K9me2 and H3K9me3) are linked to the repression of genes ${ }^{50,72}$. These histone methylations were established by the writer KMTs and can be removed by the eraser KDMs. Both methylation and demethylation of histones at specific lysine residues are important posttranslational chromatin-associated modifications ${ }^{73}$. These modifications are 
known to regulate nuclear function, including transcriptional regulation and epigenetic inheritance. Removal of repressive modifications (e.g. H3K27me3) helps to create transcriptional permissive chromatin while removing the permissive modifications (e.g., H3K4me3) contributes to the formation of repressive chromatin, suppressing gene expression ${ }^{72}$. Specifically, trimethylation at H3K4 (H3K4me3) plays a role in embryonic genome activation ${ }^{74}$. Dysfunction of $\mathrm{H} 3 \mathrm{~K} 4 \mathrm{me} 3$ by a defect of KMTs results in the down-regulation of STAT3/BIRC5, which is responsible for the extremely high apoptotic rates of blastocysts ${ }^{75}$. In both mice and human stem cells, most of the H3K4me3-regulated genes are key factors in basic biological activities and in the maintenance of stem cell pluripotency ${ }^{76,77}$. H3K4me3 markers can be removed by H3K4 histone demethylase KDM5A, which functions as a repressor by removing di- and tri-methyl groups. Loss of KDM5A has been shown to regulate cell proliferation, apoptosis and tumorigenesis in cell culture and in vivo models ${ }^{78,79}$. It has been shown that KDM5A positively regulated metastasis-related genes, including TNC, and that therapeutically targeting KDM5A inhibited tumor progression ${ }^{78}$. A study has also shown that KDM5A knockout mice displayed a mild hematopoietic phenotype, which is correlated with depression of cytokine genes ${ }^{80}$. However, unlike histone acetylation, the role of KDMs in epigenetic regulation of apoptosis during embryonic development has not been studied so far.

\section{Epigenetic cross-talk between DNA methylation and histone} modifications 
Numerous associations exist between DNA methylation and histone modification. It has been established that DNA methylation at CpG island promoter can affect methylcytosine-binding proteins (MBD1, MBD2, MBD4 and MeCP1, MeCP2) to mediate transcriptional repression during gene silencing and heterochromatin formation ${ }^{81,82}$. The $\mathrm{MBD} / \mathrm{MeCP} 1 / 2$ proteins, in turn, recruit corepressor complexes containing HDACs 1 and $2^{81}$. The growing evidence demonstrated that HDACs 1 and 2 are linked to the initiation and/ or maintenance of repression for DNA hypermethylated genes and that the simultaneous targeting of both DNA methylation and histone deacetylation leads to synergistic effects to inactive gene expression. In addition, the links between DNA methylation and histone methylation have also been demonstrated. Methylation of the histone $\mathrm{H} 3$ lysine 9 residue serves to recruit the chromatin silencer HP1 and, in this way, leads to $s$ gene repression ${ }^{83}$. The KMT has also been shown to interact with DNMT3a ${ }^{84}$ and MBD1 ${ }^{85}$. Studies have also shown that $\mathrm{KDM} 1 \mathrm{~A}$ is commonly found in association with the CoREST transcriptional repressor complex, where it interacts with HDACs1 and $2^{86,87}$. HDAC inhibitor TSA induces hyperacetylation of histones and the level of $\mathrm{H} 3 \mathrm{~K} 4 \mathrm{me} 3$, the substrate of KDM5A in the Trichomonas vaginalis ${ }^{88}$. Conversely, siRNA-mediated inhibition of KDM1A increases the abundance of H3K4ac, the substrate of HDAC ${ }^{89}$. These studies indicated that KDMs and HDACs were collaborated in establishing transcriptionally repressive chromatin and suggested that combined treatment targeting both KDM and HDAC may restore the silenced genes. In fact, studies by Huang et al. and Singh et al. have shown synergistic responses in cell proliferation in response to combined treatment with 
HDAC and KDM inhibitors ${ }^{89,} 90$. These studies suggest that combination therapy using the inhibitors of DNMT, HDAC, and KDM might be a rational approach for the prevention of FASD.

\section{Epithelial-mesenchymal transition (EMT) and neural crest cells migration}

$\mathrm{NCC}$ is a multipotent and migratory progenitor cell population that originates between the neural plate and non-neural ectoderm ${ }^{10}$. After induction at the border of the neural plate, NCCs leave their original location through a delamination process and migrate ventrally to differentiate into a diversity of neural and nonneural cell types, including neuron, glia, craniofacial cartilage, bone and connect tissue $^{91-94}$. EMT process is tightly correlated with the NCCs migration, which plays a pivotal role in craniofacial development. EMT is a process that orchestrated a change from an epithelial to mesenchyme phenotype, a process which also related to cell invasion and apoptotic resistance ${ }^{95,96}$. EMT is essential for both normal development and cancer invasion and metastasis ${ }^{97-99}$. During embryonic development, NCCs undergo an EMT and then dissociate from the neural folds and differentiate to a diversity of cell types ${ }^{10,91,93}$. EMT also plays a vital role in promoting tumor proliferation, invasion, and metastasis, exerting an anti-apoptotic effect $^{100,101}$. Studies have also shown that EMT conferred resistance to UVinduced apoptosis in three murine mammary epithelial cell lines ${ }^{102}$. Park et al. have also shown that a-mangostin can inhibit EMT and induce apoptosis in an osteosarcoma cell line ${ }^{103}$. However, the role of EMT in ethanol-induced apoptosis in NCCs and the pathogenesis of FASD remains to be defined. 
One of the well-known transcriptional factors that regulate EMT is Snail1 ${ }^{104-}$ 106. Snail1 promotes EMT primarily through the directly repressing E-cadherin, an EMT suppressing factor ${ }^{104,}$ 107. Many studies have reported that various HDAC inhibitors promote EMT in prostate cancer, head and neck squamous cell carcinoma, and colon carcinoma ${ }^{108-110}$. A study has also shown that VPA, an HDAC inhibitor, induced EMT process via the up-regulation of Snail1 ${ }^{108}$. The expression of Snail1 can be regulated by many mechanisms, including epigenetic regulation. A study by Dong Li et al. demonstrated that the H3K4me3 enrichment increased in the vicinity of the Snail1 transcriptional start site (TSS) in the process of TGF- $\beta$-induced EMT in prostate cancer cells ${ }^{111}$.

\section{Sulforaphane is an inhibitor for both DNMTs and HDACs}

Sulforaphane (SFN) is a vegetable-derived isothiocyanate that is abundant in cruciferous vegetables such as broccoli. Numerous studies have shown that SFN exhibits anti-cancer effects and prevents diabetic complications in experimental models ${ }^{112-114}$. Studies on the mechanisms underlying the anticancer activities of SFN indicate that its effects on the tumor cell cycle, apoptosis, and angiogenesis are mediated by the modulation of the related signaling pathways and genes ${ }^{115}$. Our previous studies have shown that SFN exerts an anti-apoptotic effect through upregulating antioxidant gene Nrf-2 in NCCs ${ }^{17}$. More recently, SFN is drawing great attention because of its ability to inhibit HDACs and DNMTs, two key enzymes involved in histone deacetylation and DNA methylation, respectively, to cause epigenetic modification of genes in various types of cells, including the genes involved in EMT in cancer cells ${ }^{115-117}$. SFN was found to inhibit the LPS- 
induced HDAC6, HDAC10 and DNMT3a gene expression, conferring resistance to LPS-induced apoptosis in porcine monocyte-derived dendritic cells ${ }^{118}$. Our recent studies have also shown that SFN can prevent ethanol-induced apoptosis in NCCs by reversing ethanol-induced reduction of histone acetylation at the promoter of the anti-apoptotic gene, $\mathrm{Bc} / 2^{18}$.

\section{Broccoli sprout extract (BSE)}

Recently, broccoli sprout extract (BSE) has gained a lot of attention because it can specifically deliver a reproducible and standardized dose of glucoraphanin, a precursor of SFN. The majority of SFN is formed when glucoraphanin is hydrolyzed by the plant enzyme, myrosinase, upon plant tissue damage (e.g., chopping, chewing) ${ }^{119}$. As a precursor of SFN, glucoraphanin is available in commonly consumed cruciferous vegetables. BSE is considered to have a low toxicity, and its administration in humans is well tolerated ${ }^{120-122}$. Studies have shown that dietary consumption of cruciferous vegetables, like BSE, which contains SFN, may reduce the risk of several types of cancers, including prostate, breast, lung and colorectal cancer ${ }^{119,123,124}$. One of the mechanisms through which BSE acts as a cancer chemopreventive agent is its ability to inhibit HDAC activity. It has been reported that consumption of BSE inhibited HDAC activity in peripheral blood mononuclear cells, accompanied by the induction of acetylation of histone $\mathrm{H} 3$ and $\mathrm{H} 4$ in human subjects ${ }^{125}$. Moreover, the study by Dr. Hashimoto et al. has shown that dietary intake of SFN-rich BSE was capable of preventing PCP-induced cognitive deficits and oxidative stress in mice ${ }^{126}$. Furthermore, SFN-riched BSE significantly improved social interaction, abnormal behavior, and verbal 
communication in young men with autism spectrum disorders ${ }^{120}$. Together, these results suggest that SFN-rich BSE could have potential therapeutic effects in patients with neurodevelopment problems. Thus, in the present study, I will investigate whether maternal dietary BSE can confer in vivo protection against ethanol-induced teratogenesis.

\section{B. Statement of goal}

FASD is a major development defect caused by maternal drinking alcohol during pregnancy. Growing evidence has shown that ethanol-induced excessive cell death in the specific cell population is one of the major mechanisms underlying the pathogenesis of FASD. However, there is a fundamental gap in understanding how ethanol leads to apoptotic cell death in vitro and in vivo. SFN is a chemical that is abundant in cruciferous vegetables, including broccoli, SFN is known to regulate gene expression through an epigenetic mechanism. The goal of this study is to elucidate the epigenetic mechanisms by which SFN epigenetically regulates the anti-apoptotic gene expression and prevents ethanol-induced apoptosis and teratogenesis, as well as to develop new therapeutic approaches for the prevention of FASD.

\section{Specific aims}

1. Aim1: To identify the anti-apoptotic genes that are epigenetically silenced by ethanol and de-repressed by SFN in ethanol-induced hNCCs. 
The objective of this aim is to identify the anti-apoptotic genes that are epigenetically regulated by SFN and ethanol through DNA methylation or histone modification. The sub-aims are 1) to identify the anti-apoptotic genes that are repressed by ethanol and de-repressed by SFN in hNCCs; 2) to identify the antiapoptotic genes that are hypermethylated by ethanol and de-methylated by SFN in hNCCs; and 3) to identify the anti-apoptotic genes with promoter-associated histone modifications induced by ethanol and SFN in hNCCs.

\section{Aim2: To further explore the mechanism by which SFN epigenetically} modulates the anti-apoptotic genes and apoptosis.

I expected to identify a group of anti-apoptotic genes that are epigenetically regulated by ethanol and SFN from Aim 1. The objective of this aim is to further explore the mechanism by which SFN epigenetically modulates the anti-apoptotic genes and apoptosis. The three sub-aims in this aim are 1) to determine the effects of ethanol and SFN on specific DNMT/HDAC/KDM in hNCCs; 2) to analyze the changes of DNA methylation or histone modification at the promoter of antiapoptotic genes and the expression of selected anti-apoptotic genes in hNCCs treated with ethanol or/and SFN; and 3) to determine whether SFN prevents hNCCs from ethanol-induced apoptosis by targeting specific DNMT/HDAC/KDM.

\section{Aim 3: To explore the novel therapeutic approach for the intervention of ethanol-induced teratogenesis through maternal dietary administration of SFN-rich broccoli sprout.}


Alcohol-induced apoptosis plays an important role in the pathogenesis of FASD. Therefore, a strategy for the prevention of ethanol-induced apoptosis through epigenetic modification of the anti-apoptotic genes can be very promising. The sub-aims in this aim are 1) to determine whether maternal dietary BSE can epigenetically restore the anti-apoptotic genes in ethanol exposed mouse embryos; 2) to determine whether maternal dietary BSE can prevent ethanol-induced apoptosis in mouse embryos; and 3) to determine whether dietary consumption of BSE can confer in vivo protection against ethanol-induced teratogenesis. 


\section{CHAPTER II}

\section{EXPERIMENTAL PROCEDURES}

\section{A. Cell culture and treatment}

\section{Mouse neural crest cells}

Mouse neural crest cells (JoMa 1.3 cells) were cultured on culture dishes coated with $10 \mu \mathrm{g} / \mathrm{mL}$ fibronectin. NCCs were pretreated with or without $1 \mu \mathrm{M} \mathrm{SFN}$ (LST Laboratories, St. Paul, MN) for 24 hours, followed by concurrent exposure to $1 \mu \mathrm{M}$ SFN and 50 or $100 \mathrm{mM}$ ethanol for an additional 24 hours. The stable ethanol levels were maintained by placing the cell culture dishes or plates in a plastic desiccator containing ethanol in distilled water.

\section{Human neural crest cells}

Human neural crest cells were differentiated from human embryonic stem cells (hESCs), which were purchased from WiCell ${ }^{\circledR}$ (Madison, WI, USA). hESCs were cultured in mTsSR ${ }^{\mathrm{TM}} 1$ (StemCell Technologies, Inc., Vancouver, Canada) on hESC-qualified Matrige ${ }^{\mathrm{TM}}$ (BD Biosciences, San Jose, CA) coated plates. The differentiation procedures are as follows:

1) The hESCs were first adapted to Accutase ${ }^{\circledR}$ solution and dissociated into single-cell before they were differentiated to neural crest cells. Specifically, the 
hESCs maintenance medium $\mathrm{mTsSR}^{\mathrm{TM}_{1}}$ were aspirated, and Accutase ${ }^{\circledR}$ cell dissociation reagent was added and incubated for 5-10 minutes at room temperature (RT). The colonies were gently collected once the colonies began to conform to round shape and to detach from the plate, and centrifuged the cells at room temperature for $4 \mathrm{~min}$ at $200 \mathrm{~g}$. The cells were maintained at $37^{\circ} \mathrm{C}$ in a $5 \%$ $\mathrm{CO}_{2}$ incubator and changed the medium with $\mathrm{mTsSR}{ }^{\mathrm{TM}}$ every day.

2) When hESCs plates have reached $75-85 \%$ confluence, they are ready to be passaged and differentiated into hNCCs. Aspirated hESCs maintenance medium from culture dish and add Accutase $\AA$ to detach the cells. Collected the cells in a $15 \mathrm{~mL}$ tube and centrifuged for $4 \mathrm{~min}$ at $200 \mathrm{~g}$ at room temperature. Aspirated the supernatant and resuspend the cells in a hNCCs differentiation medium (DMEM/F-12 Medium, 14.3 M L-Glutamine+ $\beta$-mercaptoethanol, MEM Non-Essential Amino Acid, $10 \mu \mathrm{g} / \mathrm{mL}$ Fgf2, $10 \mu \mathrm{g} / \mathrm{mL}$ Heregulin $\beta-1,200 \mu \mathrm{g} / \mathrm{mL}$ Long® R3-IGF1, $10 \mathrm{mM} \mathrm{CHIR} \mathrm{99021,} 10 \mathrm{mM}$ SB421542 and Penicillin and streptomycin). Upon reaching proper confluence (75\%-85\%), typically every 3-4 days, the differentiating cells should be passed using Accutase ${ }^{\circledR}$ and then maintained in hNCCs differentiation medium. The hNCCs were validated by immunocytochemistry using the NCC marker HNK1 antibody.

\section{B. Animals and treatments}

\section{Mice maintenance, time mating and BSE treatment}

C57BL/6 mice were housed in a pathogen-free barrier facility accredited by the Association for Assessment and Accreditation of Laboratory Animal Care, and 
all procedures used for this study were approved by the University of Louisville's Institutional Animal Care and Use Committee.

The female mice were acclimated to a modified liquid diet (Bioserve Inc.) containing ethanol with $5 \%$ sucrose to increase palatability. As described previously (Parnell et al., 2016), the mice were given a liquid diet containing $2.4 \%$ $(w t / v o l)$ ethanol for 2 days, then the concentration of ethanol in the diet was increased to $4.8 \%$ for 14 days. After this acclimation period, the mice were taken off the liquid diet for an abstinence period for 4 days, during which they were maintained with the standard chow and water. The mice were then bred by placing 1 or 2 females, with 1 male for a 2 hours period during the beginning of the dark cycle. The female mice were then examined for a copulation plug. The beginning of the breeding period in which a copulation plug was found was designated as the gestational day (GD) 0. SFN-rice broccoli sprout extract (BSE) was provided by Dr. Jeb Fahey in the Cullman Chemoprotection Center at The Johns Hopkins University. SFN content in this standardized, myrosinase-treated BSE is around $216 \mu \mathrm{mol}$ SFN/g powder. The amount of broccoli sprout extract to be incorporated into the liquid diet was calculated on the basis of the food intake and body weights of the animals, to give the SFN dosage of $75 \mathrm{mg} / \mathrm{kg}$ body weight/day. On GD7 and GD 8, the mice were assigned to 5 groups and placed back on the liquid diet containing: $4.8 \%$ ethanol (EtOH group), $4.8 \%$ ethanol plus BSE (EtOH+BSE group), BSE alone (BSE group), pair-fed control liquid diet (Pair-fed control group), and normal liquid diet (control group). Isocaloric amount of maltodextrin was added to BSE and control groups to control for the calories derived from the ethanol. A 
pair-fed control group was also included in this study to control for effects of the decreased diet intake observed in the EtOH group. This group of female mice was given volumes of the control liquid diet that were matched, based on milliliter diet per gram body weight, to those of the ethanol-exposed mice. During the ethanol exposure period, body weight and diet consumption were recorded every day. Pregnant mice were killed on GD 9.0, and embryos were collected for biochemical studies and the examination of apoptosis and dysmorphology. The other pregnant mice were returned to lab chow and water until GD 14 for the examination of the incidence and severity of ocular abnormalities.

\section{Fish maintenance, mating and treatment}

Zebrafish were purchased from Zebrafish International Resource Center (ZIRC; Eugene, OR) and raised and cared for by using the standard protocol ${ }^{127}$. All procedures used in this study were approved by the University of Louisville's Institutional Animal Care and Use Committee.

Zebrafish were bred and maintained under standard conditions at $28.5^{\circ} \mathrm{C}$ in a zebrafish housing system (Aquaneering; San Diego, CA). The setup was equilibrated with fish water $\left(75 \mathrm{~g} \mathrm{NaHCO}_{3}, 18 \mathrm{~g}\right.$ sea salt, $8.4 \mathrm{~g} \mathrm{CaSO}_{4}$ per 1000 $\mathrm{mL})$. Fish embryos were obtained by placing males and females in a setup box containing a plastic partition plate. The eggs were spawned 2 hours after removing the partition. Viable embryos were collected and rinsed with fish water. Then the embryos were treated with $1 \%$ ethanol with or without $1 \mu \mathrm{M}$ SFN during $3-24$ hours post-fertilization (hpf). For the KDM5A knockdown study in zebrafish 
embryos, 10 nL morpholino KDM5A was microinjected into the one-cell stage embryos.

\section{Molecular analyses}

\section{Nuclear extraction}

Nuclear extracts were isolated using the EpiQuik Nuclear Extraction Kit (Epigentek, Brooklyn, NY), following the manufacturer's protocol. Briefly, treated cells were collected and then washed with PBS, resuspended the cells with diluted NE1 (1: 10 with distilled water) and incubated on ice for $10 \mathrm{~min}$, then centrifuged the preparation for $1 \mathrm{~min}$ at 12, $000 \mathrm{rpm}$, and discarded the supernatant, followed by adding 2 volumes of NE2 to the nuclear pellets. Incubated the extract on ice for 15 min, and then sonicated for $3 \times 10$ seconds by using a Qsonica Q125 sonicator (QSonica, Newtown, CT) to increase the nuclear protein extraction. Centrifuged the suspension for $10 \mathrm{~min}$ at the maximum speed at $4^{\circ} \mathrm{C}$ and transferred the supernatant into a new tube. The protein concentration was measured by BSA Assay Reagent (Pierce, Thermo Scientific).

\section{Quantitative real-time PCR}

For quantitative real-time PCR, total RNA was isolated from the control and treated NCCs using a QIAGEN RNeasy mini kit (QIAGEN, Valencia, CA) according to the manufacturer's instruction. Total RNA was reverse transcribed using a QuantiTect Reverse Transcriptional Kit (QIAGEN, Valencia, CA) following the manufacturer's instruction. Quantitative RT-PCR was performed on a Rotor-Gene 6000 Real-time PCR system (Corbett LifeScience, Mannheim, Germany). The 
following primer sets were used for this analysis: Snail1 (Mus musculus) forward: 5'-GCCCTGCATCTGTAAGGTGT-3', reverse: 3'-CCGGGCATTGACCTCATTCT5'; E-cadherin (Mus musculus) forward: 5'-CATCGCCTACACCATCGTCA-3', reverse: 3'-CCGGGCATTGACCTCATTCT-5'; $\beta$-Actin (Mus musculus) forward: 5'CCATCCTGCGTCTGGACCTG-3', reverse: 3'-GTAACAGTCCGCCTAGAAGC-5'. KDM5A (Mus musculus) forward: 5'-AAGTTGCCTGTACGGTTGCC-3', reverse: 3'-GGAAACTAGGTAAGTGTCCCTGT-5'. Bcl2 (Mus musculus) forward: 5'TCTTTGAGTTCGGTGGGGTC -3', reverse: 3'- AGTTCCACAAAGGCATCCCAG5'. AKT1 (Mus musculus) forward: 5'- AAGGACCCTACACAGAGGCT-3', reverse: 3'- TAGGAGAACTTGATCAGGCGG-5'. BIRC5 (Mus musculus) forward: 5'CACCTTCAAGAACTGGCCCT-3', reverse: 3'- ATCGGGTTGTCATCGGGTTC-5'. BIRC6 (Mus musculus) forward: 5'- CTGTGAGTTCCTTCGGGGTT-3', reverse: 3'CTACCTGGGCTGCTGAACTC -5'. NAIP (Mus musculus) forward: 5'TTCCCCTTACAGCCACCTAAA-3', reverse: 3'- AGAGGCCTTACTCAGCTCCA5'. MCL1 (Mus musculus) forward: 5'- TGCTCCGGAAACTGGACATTA-3', reverse: 3'- GTGTTTGGCCACAAAGGCAC-5'. XIAP (Mus musculus) forward: 5'CTGGCCGGACTATGCTCATT-3', reverse: 3'- CACGATCACAGGGTTCCCAA-5'. IGF1R (Mus musculus) forward: 5'- TCCGCAACGACTATCAGCAG-3', reverse: 3'- GAAGAGTTTCCAGCCACGGA-5'. Snail1(Danio rerio) forward: 5'CAGCTTGCTACCTTCCCTTCA-3', reverse: 3'- GAACGGACGCTCACCTGTAT5'. E-cadherin (Danio rerio) forward: 5'-CAAGGTGGTCCTGAGAGTTGC-3', reverse: 3'-GAGCAGAAGAAGAGCAAGCAATAG-5'. KDM5A (Danio rerio) forward: 5'- ATGATGGAGGGAGACCTGCT-3', reverse: 3'- 
GGGCCCATCTTCAGCTTCTT-5'. AKT1 (Homo sapiens) forward: 5'CAGGATGTGGACCAACGTGA-3', reverse: 3'-AAGGTGCGTTCGATGACAGT-5'. BCl2 (Homo sapiens) forward:5'- TGGCCTTCTTTGAGTTCGGT-3', reverse: 3'ACTTGTGGCCCAGATAGGCA-5'. BIRC5 (Homo sapiens) forward: 5'AGGACCACCGCATCTCTACA-3', reverse: 3'- TTTCCTTTGCATGGGGTCGT-5'. BIRC6 (Homo sapiens) forward: 5'- GGCTAACACCAATGGAGGCT-3', reverse:3'GGAACTCATTGCATCATCCCT-5'. IGF1R (Homo sapiens) forward: 5'TCGACATCCGCAACGACTATC-3', reverse: 3'CCAGGGCGTAGTTGTAGAAGAG-5'. MCL1 (Homo sapiens) forward: 5'GACTTTTGGCCACCGGC-3', reverse: 3'- GAGAGTCACAATCCTGCCCC-5'. NAIP (Homo sapiens) forward: 5'-GCCTAGATGCAGTTCAGTTGG-3', reverse: 3'GGCACCAAAGAGGATTAGGCT-3'. XIAP (Homo sapiens) forward: 5'GGTGAAGGAGATACCGTGCG-3', reverse: 3'- GCATGTGTCTCAGATGGCCT5'. Nrf2 (Homo sapiens) forward: 5'- GAGACAGGTGAATTTCTCCCAA-3', reverse: 3'- AACGTAGCCGAAGAAACCTCA-5'. $\beta$-Actin (Homo sapiens) forward: 5'AGAAGGATTCCTATGTGGGCG-3', reverse: 3'GGATAGCACAGCCTGGATAGCA-5'. The primers were synthesized by Integrated DNA Technologies, Inc. (IDT, Coralville, IA, USA). All assays were carried out in triplicate. Relative quantitative analysis was performed by comparing the threshold cycle number for target genes and a reference $\beta$-Actin mRNA.

\section{Western blotting}

Control and treated NCCs were washed twice with iced PBS and then lysed in cold RIPA lysis buffer with $1 \mathrm{mM}$ PMSF and protease cocktail inhibitor. Whole- 
cell lysates were centrifuged at $12,000 \times g$ for $10 \mathrm{~min}$ at $4^{\circ} \mathrm{C}$, and the supernatants were used for Western blot. The protein concentration in each sample was measured by using the BCA Protein Assay Reagent Kit (Pierce, Thermo Scientific, Waltham, MA). The protein levels of Histone H3, acetyl-Histone H3, Histone H3 (tri methyl K4), cleaved caspase-3, E-cadherin, Vimentin, JARID1A, DNMT1, DNMT3A, DNMT3B, HDAC1, HDAC2, HDAC3 and $\beta$-Actin were analyzed with the following antibodies: anti-acetyl-Histone H3 rabbit pAb (06-799; Millipore, Temecula, CA). anti-Histone H3 rabbit pAb (06-755; Millipore, Temecula, CA), antiHistone H3 (tri methyl K4) rabbit pAb (ab8580, Abcam, Cambridge, MA), anticleaved caspase-3 rabbit mAb (9661, Cell Signaling, Beverly, MA, USA), anti-Ecadherin rabbit mAb (14472, Cell Signaling, Beverly, MA, USA), anti-vimentin rabbit mAb (5741, Cell Signaling, Beverly, MA, USA), anti-JARID1A rabbit mAb (3876, Cell Signaling, Beverly, MA, USA), anti-DNMT1 rabbit mAb (5032, Cell Signaling, Beverly, MA, USA), anti-DNMT3A rabbit mAb (32578, Cell Signaling, Beverly, MA, USA), anti-DNMT 3B rabbit mAb (57868, Cell Signaling, Beverly, MA, USA), anti-HDAC1 rabbit mAb (34589, Cell Signaling, Beverly, MA, USA), antiHDAC2 rabbit mAb (57156, Cell Signaling, Beverly, MA, USA), anti-HDAC3 rabbit mAb (85057, Cell Signaling, Beverly, MA, USA). anti- $\beta-A c t i n$ mouse mAb (Santa Cruz, Santa Cruz, CA), respectively. Western blot was performed by standard protocols, and the densitometry of the blot band was analyzed by ImageJ software (National Institute of Health, USA). All Western blot analyses were performed in triplicate.

\section{ChIP-qRT-PCR analysis}


ChIP assay was performed using a ChIP assay kit (Millipore, Temecula, CA) according to the manufacturer's instruction. Briefly, NCCs from control and treated groups were collected and cross-linked with $1 \%$ formaldehyde for 10 min at $37^{\circ} \mathrm{C}$, and subjected to digest with SDS lysis buffer, then sonicated to shear DNA to the length between 200 and 1000 bp using a Qsonica Q125 sonicator (QSonica, Newtown, CT). The chromatin samples were diluted with ChIP dilution buffer and immunoprecipitated using $1 \mu \mathrm{g}$ of $\mathrm{H} 3 \mathrm{~K} 4 \mathrm{me} 3$ rabbit pAb antibody (Abcam, Cambridge, MA) and mouse monoclonal (Ig)G (Santa Cruz, Santa Cruz, CA), used as a negative control. Protein A agarose beads were added for precipitation and then were washed and eluted, and DNA was purified for real-time PCR assay. For DNA purification, the DNA was first treated with proteinase $\mathrm{K}$ to remove protein and reverse the cross-links and was then purified by ChIP DNA clean \& Concentrator Kits (ZYMO Research, Irvine, CA). Quantitative real-time PCR was performed on bound and input DNAs with the following primer pairs: Snail1 (Mus musculus) P1 forward: 5'- GGCATCCCTGGGTAGTGTTTT-3', reverse: 3'GCATGTTGGCCAGAGCGAC-5'; P2 forward: GAGCCCAAGCGGAATCTCAG-3', reverse: 3'-GCATGTTGGCCAGAGCGAC-5', P3 forward: 5'- CACCTGCTCCGGTCTCAG-3', reverse: 3'GCATGTTGGCCAGAGCGAC-5', P4 forward: 5'- CAACAGTACGGTCACGCCC3', reverse: 3'-GCATGTTGGCCAGAGCGAC-5', P5 forward: 5'GCCTTGACAAAGGGGCGT-3', reverse: 3'- GTCAAAGACACCCTCGGTGG-5'. MCL1 (Homo sapiens) P1 forward: 5'- GAGAAGGCCCGAGGTGCTCATGGA-3', reverse: 3'- TCCTACGGGGTGGCGCCAGCGA-5'; P2 forward: 5'- 
GTTACGTAACCGGCACTCAGAGC-3',

reverse:

3'-

TCCTACGGGGTGGCGCCAGCGA-5;

P3

forward:

5'-

CGGGCAGCTGGTAGGTGCCGT-3',

reverse:

3'-

TCCTACGGGGTGGCGCCAGCGA-5'. IGF1R (Homo sapiens) P1 forward: 5'GGAGATGGGGGCGGCGCCTCGCA-3', reverse:

3'-

TCCTCGGGTTTTTTCCCTTTTT-5';

P2

forward:

5

CCGGTTTTTTGCGCGCGCCGGCCT-3',

reverse:

3'-

TCCTCGGGTTTTTTCCCTTTTT-5';

P3

forward:

5'-

TGCGAGCGGGCGCGTGTGCGCGGG-3',

reverse:

3'-

TCCTCGGGTTTTTTCCCTTTTT-5'. BIRC6 (Homo sapiens) P1 forward: 5'GGCCACGGCCGAACAGCTGCACC-3', reverse:

3

CCGGCGGGTGGAGCATGCGCG-5';

P2

forward:

5

GGGCGTACCGGACAGGTCGCAAG-3',

reverse:

3'-

CCGGCGGGTGGAGCATGCGCG-5';

P3

forward:

5

TGCCCCCGCGAGGAGCGGGGCGG-3',

reverse:

3'CCGGCGGGTGGAGCATGCGCG-5'. AKT1 (Homo sapiens) P1 forward: 5'GGCCGGGCTCCGTTAGCGGCCCAG-3', reverse:

3'-

TGCCCAGCGCTCGGTGCTCGGC-5';

P2

forward:

5'-

CGAGCGCGCTCCGCCCTCGCCGCC-3',

reverse:

3'-

TGCCCAGCGCTCGGTGCTCGGC-5';

P3

forward:

5'-

CGGCTCCGGGCGCGGGGCGGGCGC-3',

reverse:

3'TGCCCAGCGCTCGGTGCTCGGC-5'. BIRC5 (Homo sapiens) P1 forward: 5'AGGCCTCTCAAAGTGTTGGGATTA-3', reverse: 3'- 
GCGATTCAAATCTGGCGGTT-5',

P2

forward:

5

GGGGGCGCTAGGTGTGGGCAGGGA-3',

reverse:

3'-

GCGATTCAAATCTGGCGGTT-5';

P3

forward:

5'-

CGCGGCGGGAGGACTACAACTCCC-3',

reverse:

3'GCGATTCAAATCTGGCGGTT-5'. NAIP (Homo sapiens) P1 forward: 5'GCATGCTGTTGTGATTTTCTAGGA-3', AGATCTCTCCCTTGTGAGGGGCTT-5; TGGAAAGCACATGGCATGTCAAGG-3', AGATCTCTCCCTTGTGAGGGGCTT-5; GATTAGGGTGGGGCGCCCAACCTT-3',

P2 reverse:

3'AGATCTCTCCCTTGTGAGGGGCTT-5'. XIAP (Homo TGACCTCCCGGCCGGGGGTGGGAG-3',

P3 reverse:

3'P3 forward: 5'reverse: 3'CCCGCGGCGATCGGCTCGGTCCA-5'; P2 reverse: 3'CGCCCGGTGTCTCTTTGAGGCCCT-3', CCCGCGGCGATCGGCTCGGTCCA-5'; P3 reverse:

3'GGGCCGGCCGGTAGAGGCGCGTGA-3', reverse: 3'CCCGCGGCGATCGGCTCGGTCCA-5'

\section{Methylated Specific PCR (MSP)}

The methylation status of CpG island of all samples was determined by MSP. CpG islands were predicted by Methyl Primer Express software (Life Technologies, Grand Island, NY) using the following parameters: -CGI size 300$2000 \mathrm{bp}, \mathrm{C}+\mathrm{Gs} /$ total base $>50 \%$, CpG observed/CpG expected $>60 \%$. Genomic DNA was extracted from control and treated NCCs by using DNeasy® Blood \& 
Tissue Kit (QIAGEN, Valencia, CA) according to the manufacturer's instruction. 1 $\mu \mathrm{g}$ of genomic DNA was processed with the EpiTect® Bisulfite Kit (QIAGEN, Valencia, CA), during which the methylated DNA is protected and unmethylated cytosine is converted to uracil. The modified DNA was used as a template for PCR using primers specific for the methylated (M pair) and unmethylated sequences ( $U$ pair) (Table 1) and the methylated DNA and unmethylated DNA were amplified. The PCR products were then separated on a $2 \%$ agarose gel. When the CpG sites in the region analyzed by MSP were methylated, the methylated bands (M) presented. The unmethylated $(U)$ bands presented when the sites were unmethylated. Both $M$ and $U$ bands were presented when the sites were partially methylated. 
Table1: Primer sets of ten anti-apoptotic genes used for MSP.

\begin{tabular}{|c|c|c|c|c|}
\hline Gene & & Methylation Specific Primers & Unmethylation specific primers & $\begin{array}{l}\text { Product } \\
\text { size(bp) }\end{array}$ \\
\hline \multirow[t]{2}{*}{ AKT1 } & Forward & 5'-AGTATCGAGCGTTGGGTATC & 5'-GAGTATTGAGTGTTGGGTATT & 167 \\
\hline & Reverse & 3'-GAACGCGAACCTAACCGA & 3'-CAAACACAAACCTAACCAA & 167 \\
\hline BCL2 & Forward & 5'-TTAAAATTGTTTGTTTGCGC & 5'-ACCGTCCTAACGCTAAAATAAA & 155 \\
\hline \multirow[t]{2}{*}{ CpG 1} & Reverse & $3^{\prime}$ & & 155 \\
\hline & & AATTTAAAATTGTTTGTTTGTGT & & \\
\hline \multirow{3}{*}{$\begin{array}{l}\text { BCL2 } \\
\text { CpG2 }\end{array}$} & Forward & & 5'-ATAAGTTGTTGTAGAGGGGTTAT & 156 \\
\hline & & & & \\
\hline & Reverse & T & 3'-TC & 156 \\
\hline \multirow[t]{2}{*}{ BIRC5 } & Forward & 5'-CGCGTTTTTTGAAAGTAGTC & 5'- GTATGTGTTTTTTGAAAGTAGTT & 227 \\
\hline & Reverse & 3'-TCTAACGATTAATAACGCGC & 3'-AATCTAACAATTAATAACACACC & 227 \\
\hline \multirow[t]{2}{*}{ BIRC6 } & Forward & 5'-GTATGTATTGCGACGTCGAC & 5'- GTTGTATGTATTGTGATGTTGAT & 119 \\
\hline & Reverse & 3'-TATAACCCCCGAAATACCG & 3'-СAATATAACCCCCAAAATACCA & 119 \\
\hline IGF1R & Forward & 5'-GGTTTTACGGTCGTAACGTC & 5'-GGGTTTTATGGTTGTAATGTT & 126 \\
\hline CpG 1 & Reverse & 3'-CGTACTAAAAAACCCGAACG & 3'-ACATACTAAAAAACCCAAACA & 126 \\
\hline IGF1R & Forward & 5'-AGTTTTGGGTCGTTGTTGTC & 5'-GAGTTTTGGGTTGTTGTTGTT & 100 \\
\hline CpG 2 & Reverse & 3'-ACAATACTCCGAAAACGACG & 3'-AACAATACTCCAAAAACAACA & 100 \\
\hline \multirow[t]{2}{*}{ MCL1 } & Forward & 5'-GGTACGAGTCGGAGTTTTTC & 5'-GGGTATGAGTTGGAGTTTTTT & 192 \\
\hline & Reverse & 3'-CGATAACCTACTCCCGAAAA & 3'-ССАAТАAССТАСТСССАAАAА & 192 \\
\hline \multirow[t]{2}{*}{ NAIP } & Forward & 5'-AGGTTGGAGTATCGTGGC & 5'-TTTAGGTTGGAGTATTGTGGT & 118 \\
\hline & Reverse & 3'-GAACGTAATAACGAACGCCT & 3'- ACCAAACATAATAACAAACACCT & 118 \\
\hline \multirow[t]{2}{*}{ XIAP } & Forward & $\begin{array}{l}\text { 5'- } \\
\text { GGCGGAGGTTGTAGTGAGTC }\end{array}$ & $\begin{array}{l}\text { 5'- } \\
\text { GGAGGTGGAGGTTGTAGTGAGTT }\end{array}$ & 114 \\
\hline & Reverse & 3'-ATAAACATAAACCACCGCGC & $\begin{array}{l}\text { 3'- } \\
\text { TATAAACATAAACCACCACACCC }\end{array}$ & 114 \\
\hline
\end{tabular}

\section{6. siRNA transfection}

KDM5A siRNA (SMART pool: ON-TARGET plus mouse KDM5A), HDAC2 (SMART pool: ON-TARGET plus human HDAC2), and DNMT3A (SMART pool: ON-TARGET plus human DNMT3A) were purchased from Dharmacon (Lafayette, 
CO). Each $5 \mathrm{nmol}$ stock siRNA was reconstituted into $10 \mu \mathrm{M}$ aliquots. Scramble control siRNA (siCon) was purchased from Integrated DNA Technologies (Coralville, IA). For siRNA transfection, NCCs were seeded at 125,000 cells/per well in 12-well plates and transfected with siRNA or siCon in a final concentration of $25 \mathrm{nM}$ by using lipofectamine ${ }^{\mathrm{TM}} 2000$ (Thermo Fisher, Waltham, MA), according to manufacturer's instruction. 24 hours post-transfection, the cells were harvested at 24 hours after transfection for experiments.

\section{Analysis of apoptosis}

Apoptosis in NCCs was determined by the analysis of cleavage of caspase3 and the flow cytometry analysis of Annexin V staining. Caspase-3 cleavage was determined by Western blot, as described previously ${ }^{17}$. The number of apoptotic cells was determined by flow cytometry using a FITC Annexin $\mathrm{V}$ apoptosis detection kit (BD Bioscience, Franklin Lakes, NJ, USA), following the manufacturer's instruction. Briefly, control and treated NCCs were collected, washed twice with PBS, resuspended in binding buffer, and then incubated with Annexin $\mathrm{V}$ and $\mathrm{PI}$ for $15 \mathrm{~min}$. The apoptotic cells were detected using a FACSCalibur flow cytometer (BD Bioscience, Franklin Lakes, NJ, USA.)

The whole-mount TUNEL staining of embryos for detection and quantification of the apoptotic cells in zebrafish was performed using the TMR red in situ Detection Kit (Millipore, Temecula, CA). The embryos were fixed overnight in $4 \%$ paraformaldehyde in PBS at $4^{\circ} \mathrm{C}$. Samples were dehydrated with $100 \%$ methanol overnight, then rehydrated in successive dilutions of methanol $(100 \%$, $75 \%, 50 \%, 25 \%, 0 \%)$ in PBS. The embryos were then permeabilized with 
proteinase $\mathrm{K}(20 \mu \mathrm{g} / \mathrm{mL}$ in PBST) at room temperature for $10 \mathrm{~min}$., followed by profixation in 4\% PFA-PBS at room temperature for $20 \mathrm{~min}$. The samples were then washed five times with PBST for 5 min each and incubated in $50 \mu \mathrm{L}$ of TUNEL reaction mixture $(5 \mu \mathrm{L}$ of enzyme solution $+45 \mu \mathrm{L}$ label solution $)$ in the dark at $37^{\circ} \mathrm{C}$ for 2 hours. The reaction was stopped by washing the samples five times for $5 \mathrm{~min}$ each in PBST. Following the staining, embryos were visualized in the green channel of a fluorescent microscope (SZX16, Olympus, USA).

\section{Statistical analysis}

Statistical analyses were performed using GraphPad Prism software (GraphPad Software, San Diego, CA, USA). All data were expressed as mean \pm SEM of at least three independent experiments. Comparisons between groups were analyzed by one-way ANOVA. Multiple comparison post-tests between groups were conducted using Bonferroni's test. Differences between groups were considered significant at $p<0.05$. 


\section{CHAPTER III}

\section{SULFORAPHANE PROTECTS AGAINST ETHANOL-INDUCED APOPTOSIS IN HUMAN NEURAL CREST CELLS THROUGH DIMINISHING ETHANOL- INDUCED HYPERMETHYLATION AT THE PROMOTERS OF THE ANTI- APOPTOTIC GENES}

\section{A. Introduction}

Prenatal alcohol exposure can cause a spectrum of physical abnormalities and mental dysfunctions in children, which are defined as FASD ${ }^{128,}{ }^{129}$. Previous studies by Kotch and Sulik, as well as Dunty et al., have revealed that acute exposure to ethanol during gastrulation resulted in excessive cell death in neural crest cells (NCCs) ${ }^{14,130}$. Using a chick-specific antibody to NCCs, Cart Wright and Smith confirmed that the regions of ethanol-induced apoptosis are premigratory cranial neural crest ${ }^{131}$. Studies from our laboratory have also shown that ethanol increased apoptosis in NCCs and that ethanol-induced apoptosis in NCCs contributes heavily to ethanol-induced malformations ${ }^{132,}{ }^{133}$. However, the molecular mechanisms underlying the apoptosis that result from alcohol exposure are poorly understood. It is well-known that ethanol can induce modifications in $\mathrm{DNA}^{22-24}$ and histones ${ }^{25}, 27-29$, and such changes may mediate some detrimental effects of prenatal alcohol exposure and contribute to the abnormalities associated with FASD. An earlier study by Garro has shown that the fetal DNA was 
hypermethylated after ethanol exposure during embryonic development ${ }^{30}$. Hypermethylation in the promoter regions of genes is associated with gene repression ${ }^{134}$. DNA hypermethylation is associated with the repression of many genes involved in various cellular functions, including DNA repair, cell adhesion, and apoptosis ${ }^{40,135}$. DNA methylation has been shown to be associated with the repression of the apoptotic genes in cancer cells, which are known to avoid apoptosis $^{39,136,137}$. Hypermethylation in the Casp 8 promoter was correlated with a low Casp 8 expression, conferring apoptosis resistance in different cancer cell lines, such as pediatric cancer ${ }^{138}$, lung carcinomas ${ }^{139}$ and breast cancers ${ }^{140}$. In contrast, hypermethylation at the promoters of the anti-apoptotic genes, including $\mathrm{Bcl} 2$ is associated with the down-regulation of $\mathrm{Bcl} 2$ gene expression and apoptosis $^{141}$. These studies suggest that epigenetic regulation of apoptosis-related genes by modulating DNA methylation may contribute to the ethanol-induced apoptosis and the pathogenesis of FASD.

DNA methylation occurs on the cytosine residue of $\mathrm{CpG}$ dinucleotides through the transfer of 5-methylcytosine from the methyl donor S-adenosylmethionine (SAM) to the $\mathrm{CpG}$. The reaction is catalyzed by a family of enzymes called DNA methyltransferases (DNMT), including DNMT1, DNMT3a and DNMT3b ${ }^{142}$. DNMT1 is responsible for the maintenance of established patterns of DNA methylation, while DNMT3a and DNMT3b mediate the establishment of new or de novo DNA methylation patterns ${ }^{143,144}$. Alteration of DNA methylation by DNMT may trigger hypermethylation or hypomethylation of gene promoters and consequently result in activating or inhibiting gene expression, which can be reversed by DNMT 
inhibitor. Evidence from literature indicates that aberrant methylation of antiapoptotic genes by DNMT inhibitor may be involved in apoptosis resistance ${ }^{39,46}$. Studies have shown that loss of methylation induced by DNMT inhibitor at the promoters of the IAP gene related to apoptosis resistance ${ }^{39}$. These results indicated that DNMTi may prevent apoptosis by regulating DNA methylation at the promoters of apoptosis-related genes.

Sulforaphane (SFN) is a vegetable-derived isothiocyanate that is abundant in cruciferous vegetables such as broccoli. Our previous studies have shown that SFN prevented ethanol-induced apoptosis through upregulating the anti-oxidant gene Nrf2 in NCCs ${ }^{17}$. In addition to acting as Nrf2 inducer, SFN has been found to regulate gene expression by inhibiting the activity of DNMTs, the enzymes involved in DNA methylation ${ }^{145}$. SFN was also found to inhibit LPS-induced DNMT3a gene expression, conferring resistance to LPS-induced apoptosis in porcine monocytederived dendritic cells ${ }^{146}$.

In the present study, I tested the hypothesis that SFN can reverse ethanolinduced apoptosis through epigenetic upregulation of the anti-apoptotic genes. I observed that eight anti-apoptotic genes (AKT1, Bcl2, BIRC5, BIRC6, IGF1R, MCL1, NAIP and XIAP) are repressed by ethanol and de-repressed by SFN in hNCCs. I also found that SFN can inhibit the enzymatic activity of DNMT in ethanol exposed hNCCs. SFN also diminished ethanol-induced upregulation of DNMT3a. Among eight anti-apoptotic genes, Bcl2, XIAP, BIRC5, BIRC6, and XIAP were hypermethylated by ethanol, which can be prevented by SFN treatment. Knockdown of DNMT3a significantly reversed ethanol-induced inhibition of NAIP 
and XIAP expression and enhanced SFN's effects on the mRNA and protein expression of NAIP and XIAP in hNCCs. I also found that SFN can prevent ethanolinduced apoptosis by diminishing ethanol-induced upregulation of DNMT3a in hNCCs. These results demonstrate that SFN can prevent the ethanol-induced apoptosis by diminishing the ethanol-induced hypermethylation at the promoters of the anti-apoptotic genes in hNCC.

\section{B. Experimental procedures}

\section{Cell treatment}

hNCCs or NCCs were pretreated with or without $1 \mu \mathrm{M}$ SFN for 24 hours, followed by concurrent exposure to $1 \mu \mathrm{M}$ SFN and $50 \mathrm{mM}$ ethanol for an additional 24 hours. Cell culture dishes were placed in a plastic desiccator containing $50 \mathrm{mM}$ ethanol in distilled water to maintain the stable ethanol levels

\section{MTS cell viability assay}

Cells were seeded in a 96-well microtiter plate in a final volume of 200 $\mu \mathrm{L} /$ well. For determining the dose-response effects of ethanol on cell viability, NCCs were treated with or without 25, 50, $100 \mathrm{mM}$ ethanol for 24 hours. For determining the dose-response of SFN on ethanol-induced changes in cell viability, NCCs were treated with $0.5,1,2,4 \mu \mathrm{M}$ SFN for 24 hours, followed by 24 hours of concurrent exposure to SFN and $50 \mathrm{mM}$ ethanol. After treatment, $20 \mu \mathrm{L} /$ well MTS Reagent (Promega, Madison, WI) was added into each well and incubated for 1-4 hours at $37^{\circ} \mathrm{C}$ in standard culture conditions. The quantity of the formazan product was measured at $490 \mathrm{~nm}$ using a plate reader. 


\section{Western blot}

The protein expression levels of DNMT1, DNMT3a, DNMT3b, cleaved caspase-3, NAIP and XIAP were determined by Western blot, as described in Chapter II, C.

\section{Expression profiling of apoptosis-related genes using human apoptosis PCR array}

The human $\mathrm{RT}^{2}$ Profiler PCR array was purchased from Qiagen (Valencia, CA). RNAs were isolated from control and treated cells, then cDNA was synthesized as described in Chapter II, Section C. 2. The PCR mixture components were prepared by adding the $2 \mathrm{X} \mathrm{RT}^{2} \mathrm{SYBR}$ Green Mastermix, cDNA synthesis reaction and RNase-free water and mixing well. The PCR mixture components were dispensed into the $\mathrm{RT}^{2}$ Profiler PCR array and, then proceed the plate to the RT-PCR machine.

\section{Quantitative real-time PCR}

mRNA expression of AKT1, BCl2, BIRC5, BIRC6, IGF1R, MCL1, NAIP, XIAP and $\beta$-actin were measured by quantitative real-time RT-PCR, as described in Chapter II, Section C. 2.

\section{Analysis of DNMT activity}

hNCCs from the control and treated groups were harvested, and nuclear extracts were isolated by using the EpiQuik Nuclear Extraction Kit (Epigentek) according to the manufacturer's protocol. The protein concentrations of nuclear 
extraction were measured using the BCA protein assay kit (Thermo Scientific). 20 $\mu \mathrm{g}$ of nuclear extracts were used to determine the DNMT enzymatic activity, and the absorbance was read on a microplate reader at $450 \mathrm{~nm}$. The DNMT activity was calculated using the following formula:

DNMT activity $(\mathrm{OD} / \mathrm{h} / \mathrm{mg})=($ sample OD-Blank OD $) /(\mu \mathrm{g}$ protein amount $\mathrm{x}$ hour $)$ $x 1000$

\section{Statistical analysis}

Statistical analysis was performed as described in Chapter II, Section D.

\section{Results}

\section{Differentiation of $\mathrm{hNCCs}$ from hESCs.}

hNCCs were differentiated from hESCs using the protocol described in

Chapter II, section A. hNCCs were validated by the immunocytochemistry using the hNCC marker HNK1. The result of immunocytochemistry indicated that all hNCCs are positive for the hNCC marker HNK1 (Figure 3.1). 

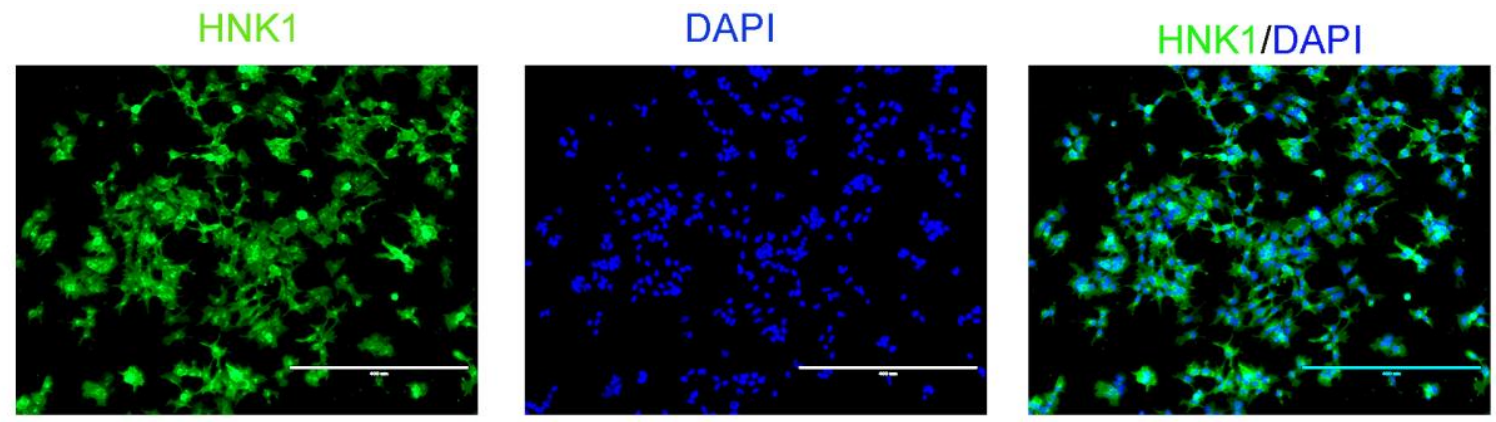

Figure 3. 1. Differentiation of hNCCs from hESCs. Immunocytochemistry analysis shows that hNCCs are positive for the NCC marker HNK1. 


\section{Ethanol treatment significantly increased apoptosis in hNCCs.}

To determine whether ethanol exposure can decrease cell viability and increase apoptosis in hNCCs, hNCCs were exposed to 25,50 or $100 \mathrm{mM}$ ethanol for 24 hours. The cell viability was analyzed using the MTS assay. As shown in Figure $3.2 \mathrm{~A}, 50$ or $100 \mathrm{mM}$ ethanol significantly reduced cell viability in hNCCs. Ethanol exposure also resulted in a dose-dependent manner, as indicated by the significant increase in caspase activation (Figure 3.2B). 

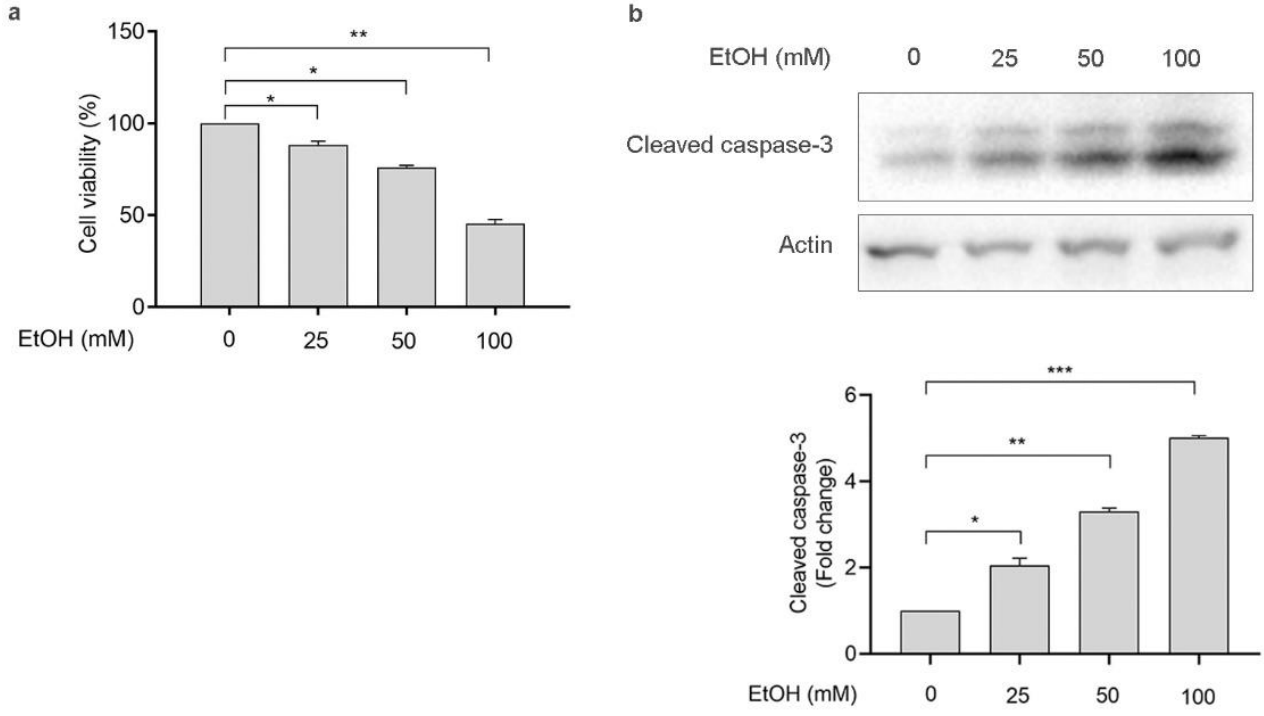

Figure 3. 2. Ethanol exposure significantly reduced cell viability and increased apoptosis in hNCCs. hNCCs were exposed to 25,50 or $100 \mathrm{mM}$ ethanol for $24 \mathrm{~h}$. Cell viability was determined by using the MTS assay (A). Apoptosis was analyzed by analysis of cleaved caspase-3 by western blotting (B). Data are expressed as a percentage of control $(A)$ or fold change over control and represent the mean \pm SEM of three separated experiments. ${ }^{*} p<0.05,{ }^{* *} p<0.01$. 


\section{SFN treatment prevented ethanol-induced apoptosis in hNCCs.}

To determine whether SFN can reduce ethanol-induced reduction in cell viability in hNCCs, the cells were treated with ethanol or/and SFN at different concentrations for 24 hours. As shown in Figure 3.3A, SFN at $1 \mu \mathrm{M}$ significantly diminished the reduction of cell viability in hNCCs exposed to $50 \mathrm{mM}$ ethanol. Treatment with SFN also significantly reduced caspase-3 activation in hNCCs exposed to ethanol (Figure 3.3B). 
a

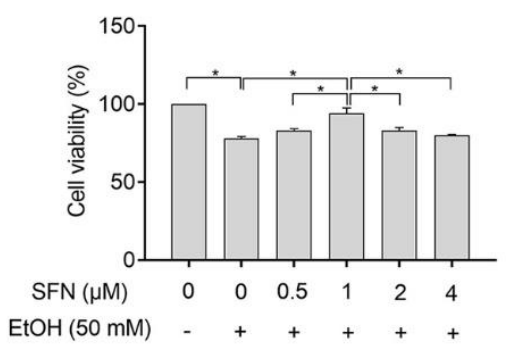

b
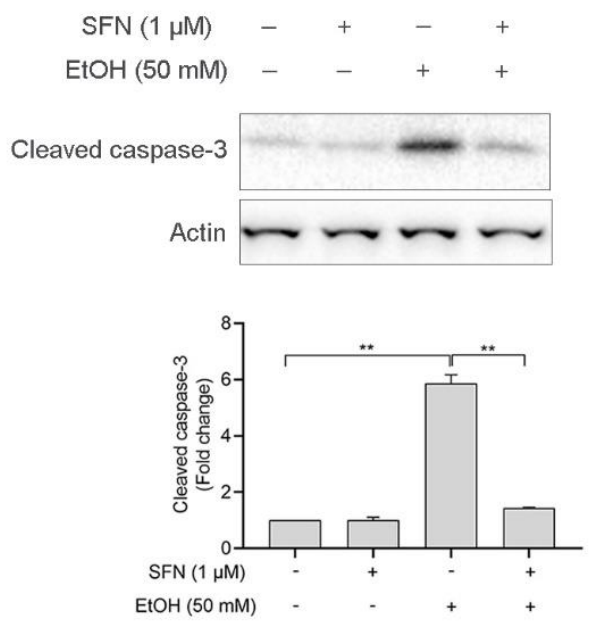

Figure 3. 3. SFN prevented the ethanol-induced reduction of cell viability and apoptosis in hNCCs. (A) hNCCs were treated with $50 \mathrm{mM}$ ethanol alone or cotreated with SFN at the indicated concentration for 24 hours. Cell viability was determined by MTS assay. (B) hNCCs were exposed to $1 \mu \mathrm{M}$ SFN for $24 \mathrm{~h}$, followed by concurrent exposure to $1 \mu \mathrm{M}$ SFN and $50 \mathrm{mM}$ ethanol for $24 \mathrm{~h}$. Apoptosis was determined by the analysis of caspase- 3 cleavage using western blotting. Data are expressed as a percentage of control $(A)$ or fold change over control (B) and represent the mean \pm SEM of three separated experiments. ${ }^{*} p<0.05,{ }^{* *} p<0.01$. 


\section{Identification of anti-apoptotic genes that are repressed by ethanol and that their repression by ethanol can be prevented by SFN in hNCCs.}

To identify the anti-apoptotic genes that were repressed by ethanol and derepressed by SFN in hNCCs, the $\mathrm{RT}^{2}$ human apoptosis array was used to profile the expression of apoptosis-related genes. The gene expression in hNCCs was depicted by the heat map with upregulation in red and downregulation in green (Figure 3.4A). The quantification of the heatmap was shown in Figure 3.4B. We have identified eight anti-apoptotic genes that were down-regulated by ethanol and that their down-regulation can be prevented by SFN, including AKT1, BCL2, BIRC5, BIRC6, IGF1R, MCL1, NAIP and XIAP. The results from human apoptosis array were validated by quantitative RT-PCR (Figure 3.4C). 

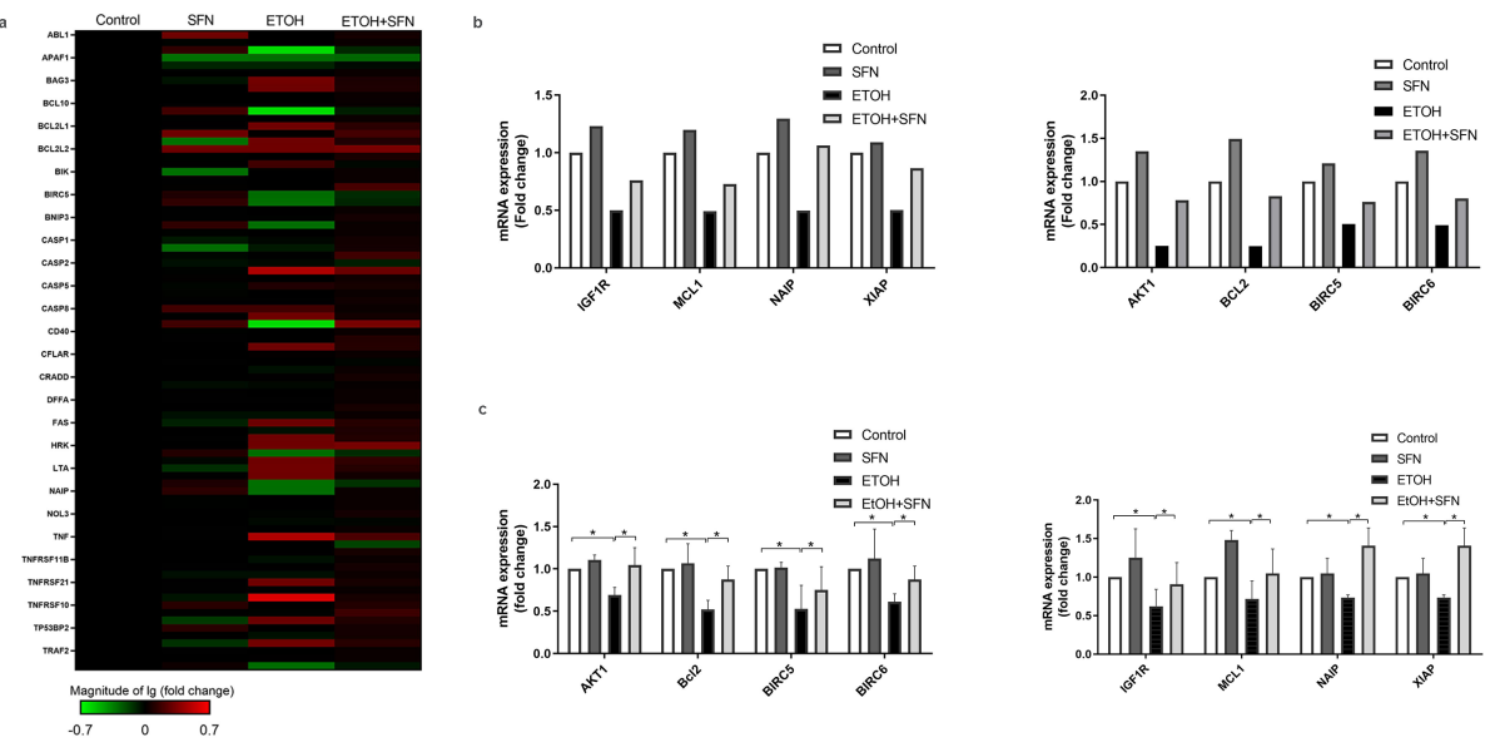

Figure 3. 4. Identification of the anti-apoptotic genes that are repressed by ethanol and de-repressed by SFN. hNCCs were pre-treated with $1 \mu \mathrm{M}$ SFN for 24 hours and then co-treated with $1 \mu \mathrm{M}$ SFN and $50 \mathrm{mM}$ ethanol for another 24 hours. The $R T^{2}$ apoptosis array was used to profile the apoptosis-related genes expression in control and treated hNCCs, and the mRNA expression of 84 apoptosis-related genes was depicted by the heat map (A). Eight anti-apoptotic genes were identified from apoptosis array analysis (B). The mRNA expression of the selected antiapoptotic genes was validated using RT-PCR (C). The data were expressed as fold change over control and represent the mean \pm SEM of three separated experiments. ${ }^{*} p<0.05$. 


\section{SFN diminished ethanol-induced increase in DNMT activity and up- regulation of DNMT3a in hNCCs.}

To determine the effects of ethanol and SFN on the activity of DNMT, hNCCs were pretreated with SFN for 24 hours, then co-treated with SFN and ethanol for another 24 hours. As shown in Figure 3.5A, treatment with SFN significantly reduced the ethanol-induced increase in the activity of DNMT in hNCCs. Three enzymatically active DNMTs, includingDNMT1, DNMT3a and DNMT3b, were involved in DNA methylation in eukaryotic cells ${ }^{38,147}$. We thus examined the protein expression of DNMT1, DNMT3a and DNMT3b by Western blot in hNCCs. As shown in Figure 3.5B, while ethanol exposure did not significantly change the protein expression of DNMT1 and DNMT3b, ethanol exposure significantly increased the expression of DNMT3a in hNCCs. Cotreatment with SFN and ethanol reduced ethanol-induced increase in DNMT3a expression. This result suggested that DNMT3a may be the primary methyltransferase responsible for ethanol-induced hypermethylation at the promoters of anti-apoptotic genes in hNCCs. 


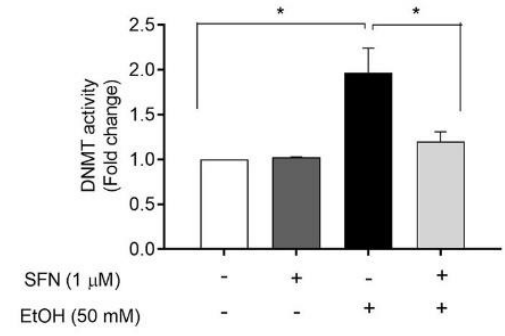

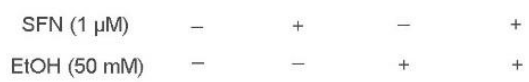
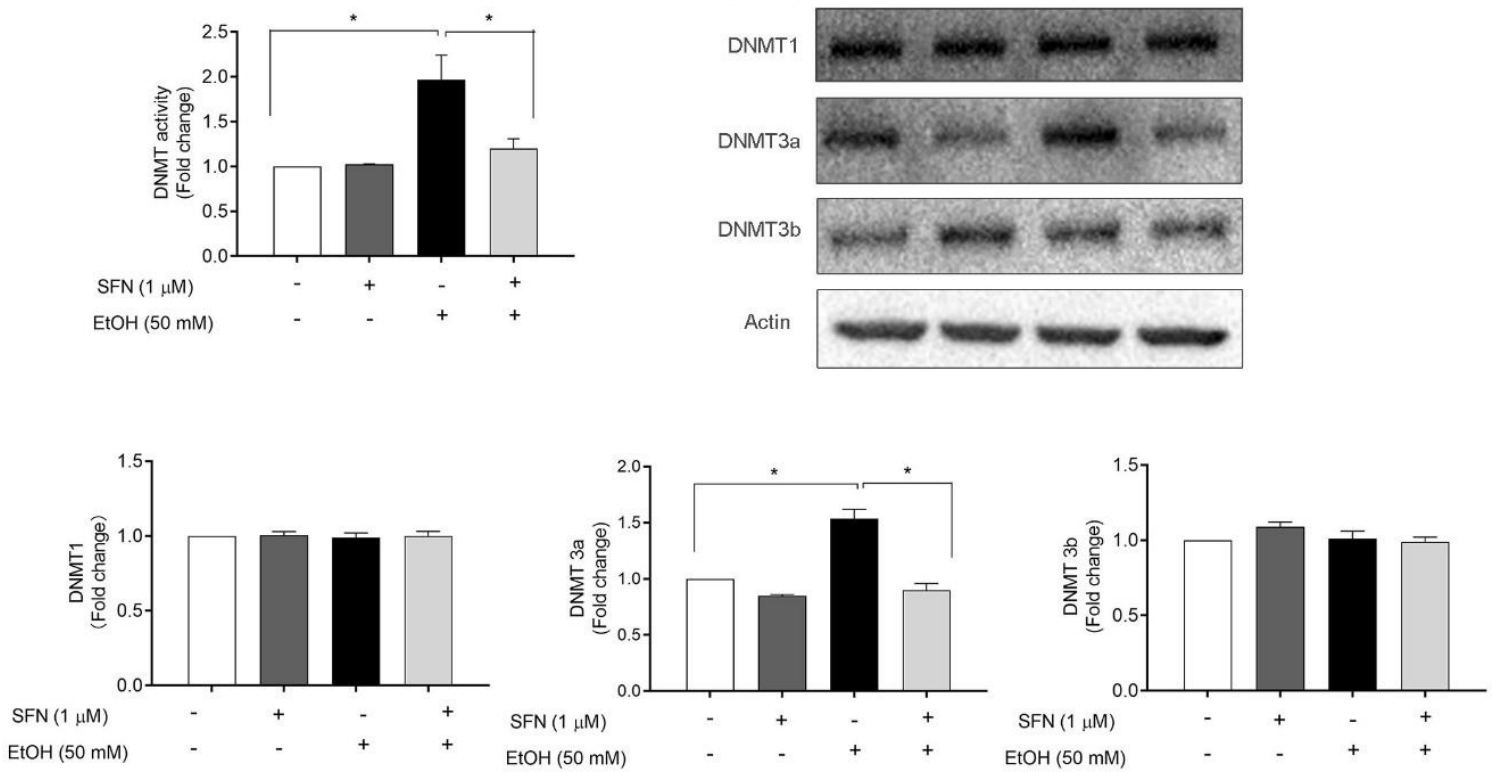

Figure 3. 5. Co-treatment with SFN and ethanol reduced DNMT activity and DNMT3a expression in ethanol exposed hNCCs. hNCCs were treated with $1 \mu \mathrm{M}$ SFN for 24 hours, then co-treated with $1 \mu \mathrm{M}$ SFN and $50 \mathrm{mM}$ ethanol for an additional 24 hours. (A) Treatment SFN significantly reduced the ethanol-induced increase in the activity of DNMT in hNCCs (B) Co-treatment with SFN and ethanol significantly decreased the ethanol-induced increase in DNMT3a protein expression in hNCCs. Data are expressed as fold change over control and represent the mean \pm SEM of three separated experiments. ${ }^{*} p<0.05$. 


\section{SFN diminished the ethanol-induced hypermethylation at the}

promoters of the selected anti-apoptotic genes in hNCCs.

To determine whether ethanol-induced up-regulation of DNMT3a and increase in DNMT activity can result in hypermethylation at the promoters of the selected anti-apoptotic genes and whether SFN that can prevent ethanol-induced up-regulation of DNMT3a and increase in DNMT activity can diminish ethanolinduced hypermethylation at the promoters of anti-apoptotic genes, the methylation of $\mathrm{CpG}$ islands at the promoters of the identified anti-apoptotic genes in human NCCs was analyzed by using methylation-specific PCR (MSP). Exposure to ethanol resulted in a significant increase in the methylation level at the promoters of Bcl2, XIAP, BIRC5, BIRC6 and NIAP. Co-treatment with SFN significantly diminished ethanol-induced hypermethylation at the promoters of these anti-apoptotic genes in human NCCs (Figure 3.6). These results demonstrated that ethanol exposure can induce hypermethylation at the promoters of the selected anti-apoptotic genes in hNCCs, which can be prevented by cotreatment with SFN. 
a

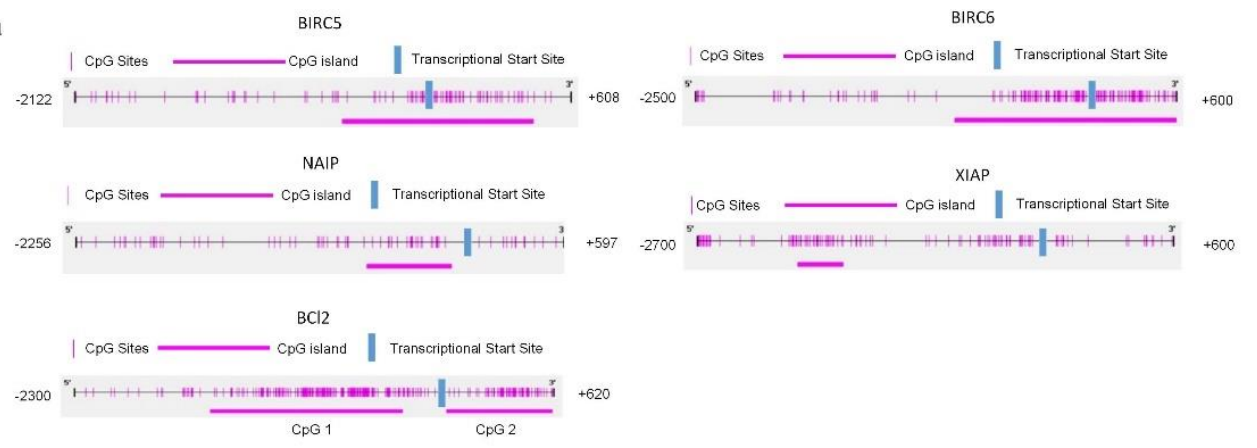

b
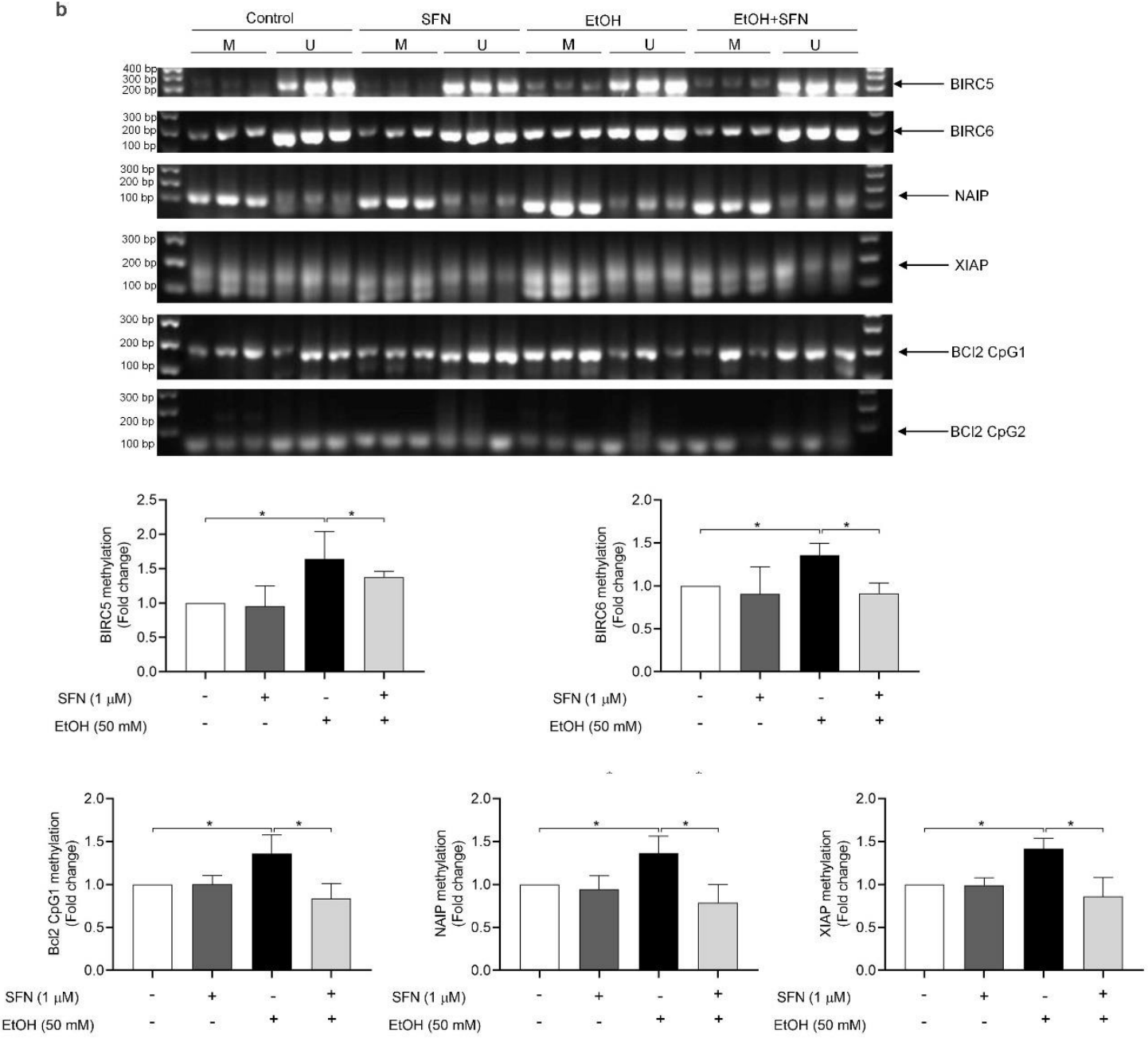
Figure 3. 6. SFN significantly diminished the ethanol-induced hypermethylation at the promoters of Bcl2, XIAP, BIRC5, BIRC6 and NAIP in hNCCs. hNCCs were pre-treated with $1 \mu \mathrm{M}$ SFN for 24 hours and then continue to be exposed in medium containing SFN, ethanol, or in combination for 24 hours. (A) Schematic illustration of the $\mathrm{CpG}$ sites and $\mathrm{CpG}$ island in the promoter of the anti-apoptotic gene. (B) DNA methylation status at the $\mathrm{CpG}$ island of the selected anti-apoptotic genes was determined by using MSP. Data are expressed as fold change over control and represent the mean \pm SEM of three separated experiments. ${ }^{*} p<0.05$. M: methylated DNA, U: unmethylated DNA. 


\section{Knockdown of DNMT3a and SFN treatment significantly diminished ethanol-induced down-regulation of NAIP and XIAP in hNCCs}

To further confirm that DNMT3a is responsible for the ethanol-induced hypermethylation at the promoters of anti-apoptotic genes and subsequent downregulation of these genes, DNMT3a was knocked down by siRNA in hNCCs before treatment with ethanol and SFN. We found that ethanol exposure significantly decreased mRNA expression of NAIP and XIAP. Protein expression of NAIP and XIAP was also decreased in ethanol-exposed hNCCs. The knockdown of DNMT3a or treatment with SFN significantly diminished ethanol-induced decreases in the mRNA and protein expression of NAIP and XIAP. In addition, knockdown of DNMT3a enhanced the effects of SFN on the mRNA and protein expression of NAIP and XIAP (Figure 3.7A, B). These results indicated that either knockdown of DNMT3a or inhibition of DNMT activity by SFN can diminish ethanol-induced repression of anti-apoptotic genes in hNCCs. 

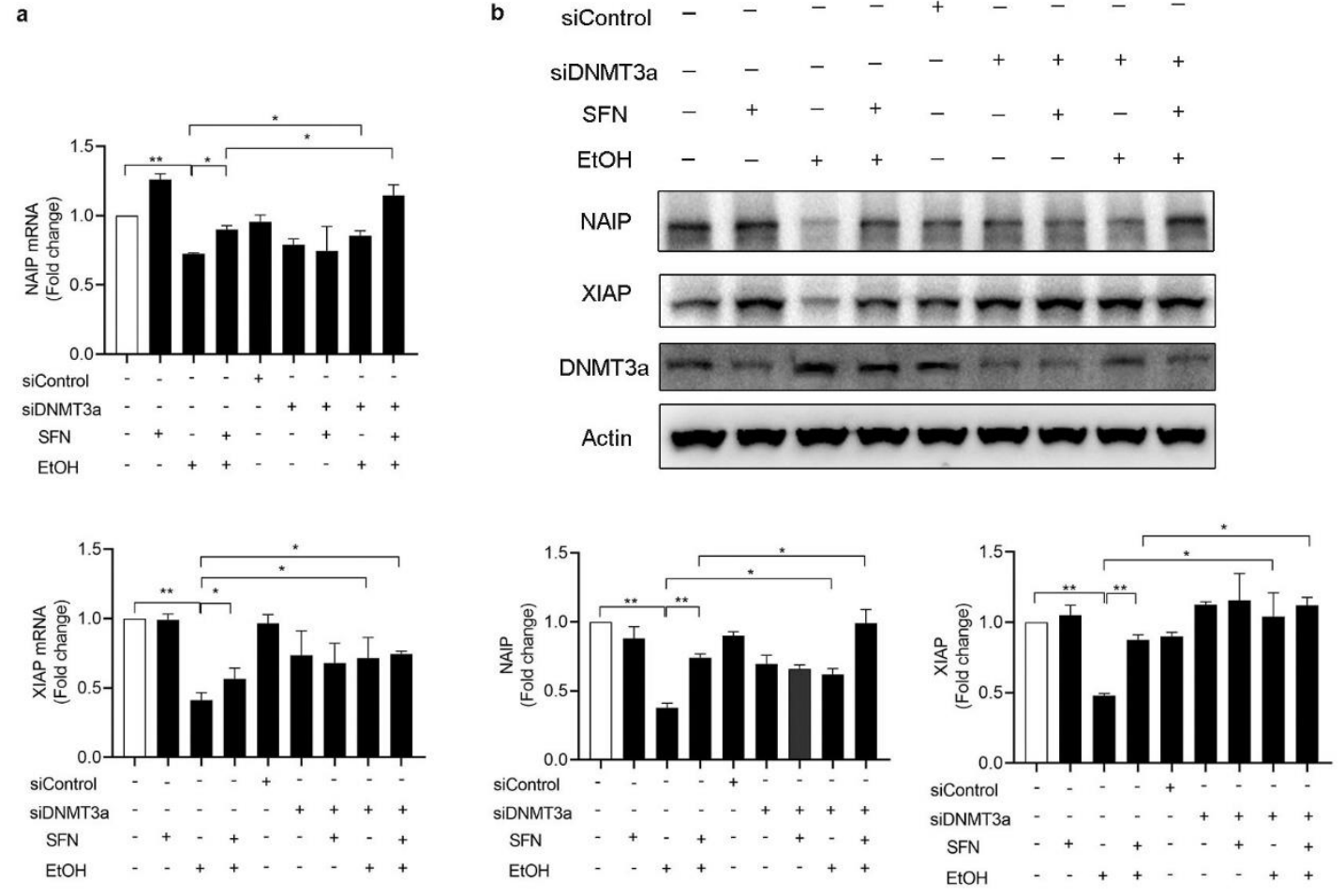

Figure 3. 7. Knockdown of DNMT3a and SFN treatment significantly diminished ethanol-induced down-regulation of NAIP and XIAP in hNCCs. hNCCs transfected with control-siRNA or DNMT3A-siRNA were treated with $1 \mu \mathrm{M}$ SFN or $50 \mathrm{mM}$ ethanol or co-treated with ethanol and SFN for further 24 hours. (A) The mRNA expression of NAIP and XIAP was determined by quantitative RT-PCR. (B) The protein expression of NAIP and XIAP was determined by western blotting. Data are expressed as fold change over control and represent the mean \pm SEM of three separated experiments. ${ }^{*} p<0.05,{ }^{* *} p<0.01$. 


\section{Knockdown of DNMT3a and SFN treatment prevented ethanol-induced apoptosis in hNCCs.}

To determine whether ethanol-induced up-regulation of DNMT3a and subsequent hypermethylation at the promoters of the selected anti-apoptotic genes and repression of these genes contribute to ethanol-induced apoptosis and whether SFN can prevent ethanol-induced apoptosis by diminishing ethanolinduced up-regulation of DNMT3a, DNMT3a was knocked down by siRNA in hNCCs. Apoptosis was determined by the analysis of caspase-3 activation using western blot. I found that ethanol exposure significantly increased apoptosis in hNCCs. The knockdown of DNMT3a by siRNA or down-regulation of DNMT3a by SFN significantly diminished ethanol-induced apoptosis in hNCCs (Figure 3.8). These results demonstrate that ethanol-induced up-regulation of DNMT3a contributes to ethanol-induced apoptosis and that SFN can prevent ethanolinduced apoptosis in hNCCs by diminishing ethanol-induced up-regulation of DNMT3a. 

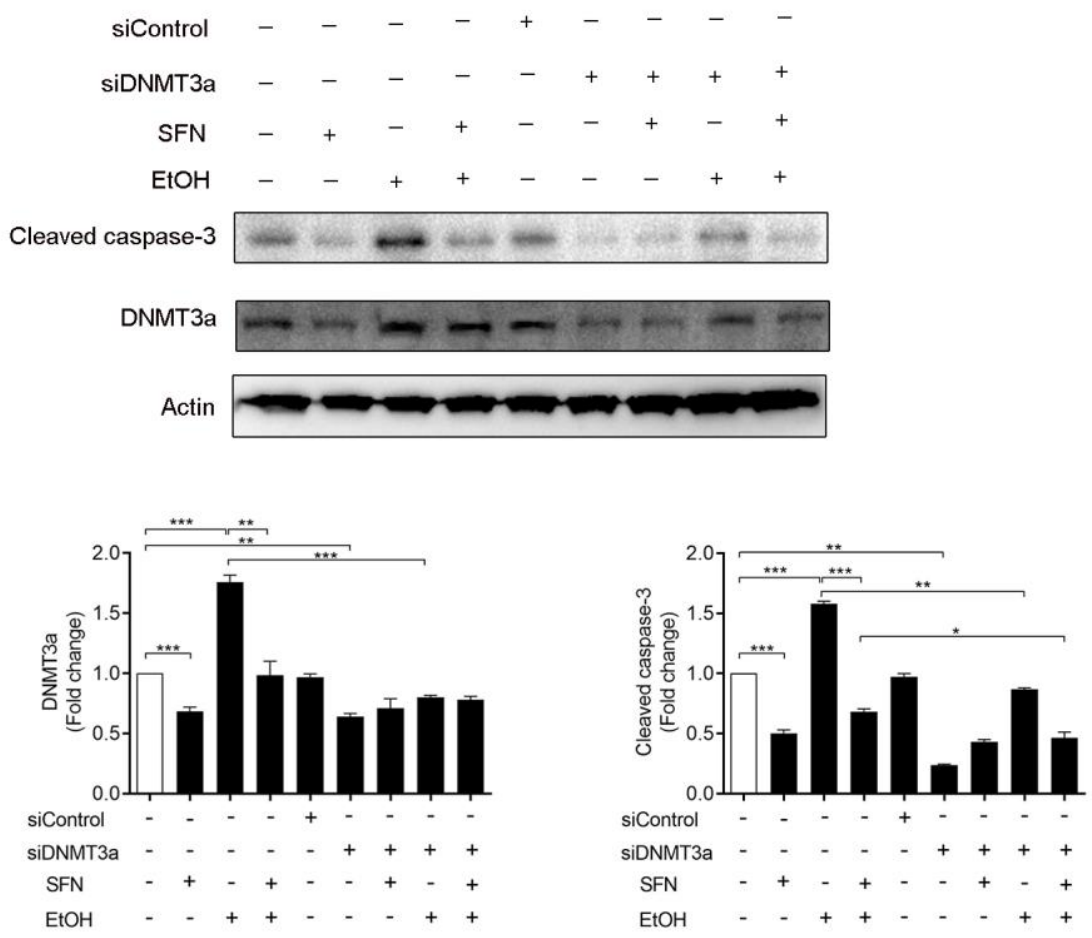

Figure 3. 8. Knockdown of DNMT3a and SFN treatment prevented ethanolinduced apoptosis in hNCCs. hNCCs transfected with siRNA-DNMT3a or siRNAcontrol were treated with1 $\mu \mathrm{M}$ SFN or $50 \mathrm{mM}$ ethanol or co-treat with SFN and ethanol for 24 hours. The protein expression of DNMT3a and cleaved caspase-3 were determined by western blotting. Data are expressed as fold change over control and represent the mean \pm SEM of three separated experiments. ${ }^{*} p<0.05$, ${ }^{* *} p<0.01,{ }^{* * *} p<0.001$. 


\section{Discussion}

Ethanol-induced apoptosis in hNCCs is one of the major mechanisms underlying the pathogenesis of FASD. Studies have shown that epigenetic modifications contribute to the harmful consequences of alcohol abuse during pregnancy in the developing fetus ${ }^{35}$. Epigenetic modifications, such as altered DNA methylation and histone modifications, are thought to influence apoptosisrelated genes in response to ethanol exposure. Recent studies in our laboratory have demonstrated that ethanol exposure significantly increased apoptosis through decreasing histone acetylation on the promoter of the anti-apoptotic gene, $\mathrm{Bcl}{ }^{18}$. However, the role of DNA methylation in the ethanol-induced apoptosis has not been well elucidated. In this study, I determined whether ethanol exposure can increase apoptosis through epigenetically repressing the anti-apoptotic genes and whether SFN, a well-known epigenetic regulator, can epigenetically de-repress the anti-apoptotic gene expression. I found that treatment with ethanol significantly repressed the expression of AKT1, Bcl2, BIRC5, BIRC6, IGF1R, MCL1, NAIP and XIAP. SFN treatment diminished ethanol-induced repression of these genes in hNCCs. Treatment with SFN also significantly reduced ethanol-induced increase in the enzymatic activity of DNMT in hNCCs. In addition, SFN treatment significantly reduced the ethanol-induced hypermethylation at the promoters of BIRC5, BIRC6, NAIP, XIAP and Bcl2, suggesting that the aberrant DNA methylation induced by ethanol contributes to the downregulation of the antiapoptotic genes in ethanol-exposed hNCCs. 
To further explore the molecular mechanisms by which ethanol induces the aberrant methylation at the promoters of the anti-apoptotic genes, I analyzed the protein levels of DNMT1, DNMT3a and DNMT3b, which are the main enzymes involved in DNA methylation in mammals ${ }^{142}$. DNMT1 contributes to maintaining the established DNA methylation patterns. DNMT3a and DNMT3b are known as de novo methyltransferase and play a role in the establishment of methylation patterns during development ${ }^{148}$. In addition, the defect of DNMT3a was reported to result in nonprogressive neurodevelopmental conditions ${ }^{149}$. Interestingly, environmental stimuli, like stress, in the pregnant rat has been found to increase DNMT3a expression and alter DNA methylation levels in their offspring ${ }^{150}$. Study has also demonstrated that ethanol exposure increased the expression of DNMT3a in rats ${ }^{151}$. Consistent with these findings, I observed that ethanol treatment increased the expression of DNMT3a, which can be diminished by treatment with SFN in hNCCs, indicating that upregulation of DNMT3a contributes to ethanol-induced hypermethylation at the promoters of anti-apoptotic genes and that SFN can diminish ethanol-induced hypermethylation at the promoters of antiapoptotic genes by reducing ethanol-induced up-regulation of DNMT3a. Our observation is consistent with the study from $Q u X$ et al., which has shown that SFN decreased DNMT3a gene expression in porcine monocyte-derived dendritic cells ${ }^{146}$.

Studies from others have demonstrated that DNMT3a can regulate apoptosisrelated genes expression in cancer cells through changing the methylation level at the promoter region of the apoptosis-related genes, such as Bad, Bax and Bcl2 ${ }^{152-}$ 
154. In the present study, I have found that knockdown of DNMT3a by transfected with siRNA-DNMT3a significantly reduced the ethanol-induced down-regulation of NAIP and XIAP. The knockdown of DNMT3a also enhanced SFN's effects on promoting the expression of NAIP and XIAP in ethanol-exposed hNCCs. These results suggest that the upregulation of DNMT3a contributes to ethanol-induced down-regulation of anti-apoptotic genes, NAIP and XIAP, by inducing hypermethylation at the promoters of these genes.

It has been reported that DNMT inhibitor-induced aberrant methylation at the promoters of the anti-apoptotic genes, including ciAP1, ciAP2, Survivin, NAIP and $\mathrm{XIAP}$, might be related to apoptosis resistance in cancer cells ${ }^{39}$. As a DNMT inhibitor, SFN has also been shown to promote cell survival in a dose and cell typedependent manner. For instance, the study by $\mathrm{Qu}$ et al., indicated that SFN suppressed the LPS-induced apoptosis in monocyte-derived dendritic cells through down-regulating DNMT3a and the suppression of TGF- $\beta$ signaling ${ }^{146}$. Additionally, previous studies in our laboratory have demonstrated that SFN can promote cell survival by upregulating the anti-apoptotic gene, Bcl2, in ethanol exposed NCCs ${ }^{18}$. Treatment with SFN also significantly diminished the ethanolinduced reduction of Snail1, conferring resistance to apoptosis in NCCs ${ }^{133}$. In the present study, I found that knockdown of DNMT3a significantly decreased caspase-3 activation in ethanol-exposed hNCCs and enhanced SFN's protective effects on ethanol-induced apoptosis, suggesting that inhibition of DNMT3a contributes to SFN's protective effects on the ethanol-induced apoptosis in hNCCs. 
In summary, the present study indicated that ethanol treatment can inhibit the expression of the anti-apoptotic genes, including AKT1, Bcl2, BIRC5, BIRC6, IGF1R, MCL1, NAIP and XIAP and that ethanol-induced repression of these antiapoptotic genes can be prevented by SFN. I also observed that SFN diminished ethanol-induced up-regulation of DNMT3a and an increase in the enzymatic activity of DNMT. Ethanol exposure also resulted in hypermethylation at the promoters of the selected anti-apoptotic genes, including BIRC5, BIRC6, NAIP, XIAP and Bcl2, which can be prevented by SFN treatment. Knockdown of DNMT3a and SFN also significantly diminished the ethanol-induced down-regulation of NAIP and XIAP, and subsequently conferring protection against ethanol-induced apoptosis in hNCCs. These findings suggest that SFN may represent a promising and effective agent for the prevention of ethanol-induced apoptosis and FASD. 


\section{CHAPTER IV}

\section{SULFORAPHANE PREVENTS HUMAN NEURAL CREST CELLS FROM}

ETHANOL-INDUCED APOPTOSIS THROUGH DIMINISHING ETHANOL-

\section{INDUCED REDUCTION OF HISTONE ACETYLATION AT THE \\ PROMOTER OF THE ANTI-APOPTOTIC GENES}

\section{A. Introduction}

Maternal ethanol exposure during pregnancy could result in physical and behavioral abnormalities in the offspring, which is defined as fetal alcohol spectrum disorder $(F A S D)^{128}, 129,155$. Exposure of mouse or chicken embryos to ethanol during a certain period of development can also cause craniofacial abnormalities associated with FASD ${ }^{14,131,156}$. Extensive research with mice or chicken embryos revealed that exposure to ethanol could induce excessive cell death in neural crest cells (NCCs) ${ }^{130,157,158}$. Studies in our lab also shown ethanol exposure increased apoptosis in NCCs and that ethanol-induced apoptosis in NCCs contributes heavily to ethanol-induced malformations ${ }^{132,}{ }^{133}$. The molecular mechanisms underlying FASD that result from prenatal alcohol exposure are poorly understood. Studies have shown that ethanol can induce modifications in $\mathrm{DNA}^{22-24}$ and histones ${ }^{25,27-29}$, and such changes may mediate the detrimental effects of prenatal alcohol exposure and contribute to the abnormalities associated with FASD. The first study on the role of histone modification in alcohol-induced damages was conducted on 
liver cells. This study found that H3K9ac was increased in a time- and dosedependent manner after ethanol exposure ${ }^{159,160}$. Furthermore, it has also been reported that chronic alcohol exposure in humans resulted in global increases in the levels of histone 3 at lysine $4(\mathrm{H} 3 \mathrm{~K} 4 \mathrm{me} 3)^{161}$. Histone acetylation and methylation play a critical role in the regulation of the expression of the genes involved in cell cycle progression and apoptosis ${ }^{162,163}$. For instance, study has shown that prenatal alcohol exposure decreased histone H3K9 acetylation at the promoter regions of the anti-apoptotic gene $\mathrm{Bcl} 2$, resulting in the downregulation of $\mathrm{Bcl} 2$ and apoptosis in fetal myocyte ${ }^{164}$. Taken together, these works indicated that ethanol-induced epigenetic modulation of apoptosis-related genes could be an important mechanism underlying the pathogenesis of FASD.

The complexes of nuclear DNA and proteins are called chromatin. In chromatin, the fundamental repeat unit is the chromosome, consisting of one octamer comprised of four core histone proteins (H2A, H2B, H3 and H4) around which 147 base pairs of DNA are wrapped ${ }^{48}$. Each of the core histones has an Nterminal "tail" that can be subject to several covalent modifications associated with euchromatic (open) and heterochromatin (close) chromatin states. These covalent modifications are established by "writers", such as histone lysine methyltransferases (KMTs), histone acetyltransferases (HATs). These modifications can be removed by "erasers," such as histone demethylases (KDMs), histone deacetylases (HDACs) ${ }^{165}$. HATs and HDACs are associated with histone acetylation and deacetylation, representing transcriptional activation or repression, respectively ${ }^{166,167}$. There are 18 HDACs in human, and they are distributed into 4 
classes: class I HDACs (1, 2, 3 and 8), class II HDACs (4, 5, 6, 7 and 9), and class IV HDACs are all dependent upon zinc for activity, whereas class III HDACs are structurally unique because they need NAD+ for enzymatic activity ${ }^{168,} 169$. The histone deacetylase inhibitor (HDACi) can interfere with the deacetylase function of HDAC, improving the acetylation of histone and activating gene expression. Substantial studies have indicated that the HDACi are emerging as a prominent class of therapeutic agents for the treatment of a variety of cancers, such as pancreatic, ovarian, breast, colon, prostate, and thyroid cancer ${ }^{59-62}$. Other studies have also demonstrated that HDACi has important neuroprotective effects in the prevention or treatment of nervous system diseases ${ }^{64-66}$. HDACi is also known to inhibit apoptosis, increase cell survival and enhance anti-inflammatory response ${ }^{64,}$ 65, 68, 69. The study from Dr. Zhang has shown that the HDACi valproic acid (VPA) protected against neuronal apoptosis by activating BNDF-TrkB signaling at the transcriptional level, VPA treatment also resulted in the hyperacetylation of histone H3K14 at the promoter regions of BNDF, and promoted transcriptional activity ${ }^{71}$. VPA has also been shown to upregulate the expression of anti-apoptotic gene Hsp70 by enhancing the acetylation of histone $\mathrm{H} 3$ at Hsp70 promoter ${ }^{68}$. These results demonstrate that HDACis might be used as protective agents to regulate the apoptosis-related genes and prevent ethanol-induced apoptosis.

Sulforaphane (SFN) is a vegetable-derived isothiocyanate that is abundant in cruciferous vegetables such as broccoli. Compelling evidence indicated that SFN can prevent cancer and other diseases through its regulatory role on the tumor cell cycle, apoptosis and angiogenesis ${ }^{112,170-172}$. Interestingly, our previous studies 
have shown that SFN exerts an anti-apoptotic effect through upregulating the antioxidant gene Nrf2 in $\mathrm{NCCs}^{17}$. Recent studies have shown that SFN can inhibit HDACs, the enzyme involved in histone deacetylation ${ }^{115,117,173}$. Moreover, some other study has shown the metabolite of SFN, SFN-cysteine, can interact with the active site of HDAC, which subsequently inhibits HDAC activity ${ }^{174}$. SFN was found to inhibit LPS-induced HDAC6, HDAC10 and DNMT3a gene expression, conferring resistance to LPS-induced apoptosis in porcine monocyte-derived dendritic cells ${ }^{146}$. Our recent study has also shown SFN can prevent ethanolinduced apoptosis in NCCs by diminishing ethanol-induced reduction of histone acetylation at the promoter of the anti-apoptotic gene, Bcl $2^{18}$. We have also demonstrated that SFN treatment prevented the ethanol-induced reduction of H3K4me3 on the promoters of Snail1, a key transcriptional factor of epithelial to mesenchymal transition (EMT), and prevented ethanol-induced apoptosis in $\mathrm{NCCs}^{133}$.

In the present study, I tested the hypothesis that SFN can reverse ethanolinduced apoptosis through epigenetic upregulation of the anti-apoptotic genes. I found that SFN can diminish the ethanol-induced increase in the enzymatic activity of HDAC and up-regulation of HDAC2. Treatment with SFN also diminished ethanol-induced reduction of $\mathrm{H} 3$ acetylation at the promoters of AKT1, BIRC6 and XIAP, resulting in increased expression of AKT1, BIRC6 and XIAP in ethanol exposed hNCCs. Knockdown of HDAC2 can also prevent ethanol-induced repression of these genes, enhancing SFN's protective effects on the expression of anti-apoptotic genes in ethanol-exposed hNCCs. I have also demonstrated that 
SFN and knockdown of HDAC2 can protect hNCCs against ethanol-induced apoptosis. These results demonstrate that SFN can significantly prevent ethanolinduced apoptosis in hNCCs through epigenetically upregulating the anti-apoptotic genes.

\section{B. Experimental Procedures}

\section{Cells treatments}

hNCCs were pretreated with or without $1 \mu \mathrm{M}$ SFN for 24 hours, followed by concurrent exposure to $1 \mu \mathrm{M} \mathrm{SFN}$ and $50 \mathrm{mM}$ ethanol in a plastic desiccator containing $50 \mathrm{mM}$ ethanol in distilled water.

\section{ChIP-qRT-PCR analysis}

ChIP experiments were performed as described in Chapter II, C. 4. Chromatin was immunoprecipitated with antibodies to H3ac. The qPCR was carried out with primers shown in Chapter II, C.4. $\mu \mu$

\section{Quantitative real-time PCR}

mRNA expression of AKT1, BIRC6, and XIAP were measured by quantitative RT-PCR, as described in Chapter II, Section C. 2.

\section{Western Blotting}

The protein level of H3Ac, Cleaved caspase-3, HDAC1, HDAC2, HDAC3, Histone $\mathrm{H} 3, \mathrm{AKT1}, \mathrm{BIRC6}$, and XIAP were determined by western blotting as described in Chapter II, Section C. 3.

\section{Analysis of HDAC activity}


The nuclear protein was extracted from the control and treated hNCCs by using the EpiQuik Nuclear Extraction Kit (Epigentek) according to the manufacturer's protocol. The HDAC activity was measured using an HDAC fluorometric activity assay kit (Enzo), in which an established HDAC inhibitor TSA was taken as a control. $10 \mu \mathrm{g}$ of nuclear extract was incubated with or without inhibitor for 1 hour at $37^{\circ} \mathrm{C}$ and then stoped by the addition of the developer for further 10-15 min. The absorbance was read on fluorimeter at $360 \mathrm{~nm}$ excitation and $460 \mathrm{~nm}$ emission wavelength.

\section{HDAC2 siRNA transfection}

For HDAC2 siRNA transfection, hNCCs were transfected with HDAC2 siRNA or scrambled control siRNA in a final concentration of $25 \mathrm{nM}$ as described in Chapter II, Section C. 7

\section{Statistical analysis}

Statistical analysis was performed as described in Chapter II, Section D.

\section{C. $\underline{\text { Results }}$}

1. SFN diminished the ethanol-induced increase HDAC activity and the up-regulation of HDAC2 in hNCCs.

To determine the effects of ethanol and SFN on HDAC activity, hNCCs were pretreated with or without $1 \mu \mathrm{M}$ SFN for 24 hours, followed by concurrent exposure to $1 \mu \mathrm{M}$ SFN or $50 \mathrm{mM}$ ethanol for additional 24 hours. We found that exposure to ethanol alone for 24 hours significantly increased the activity of HDAC in hNCCs. 
Co-treatment with SFN significantly reduced the ethanol-induced increase in the activity of HDAC. In addition, ethanol exposure resulted in a significant increase in the protein expression of HDAC2 in hNCCs. Treatment with SFN dramatically reduced the up-regulation of HDAC2 in hNCCs exposed to ethanol (Figure 4.1A, B). These results suggest that HDAC2 may contribute to the ethanol-induced reduction of histone acetylation at the promoters of the anti-apoptotic genes in hNCCs. 


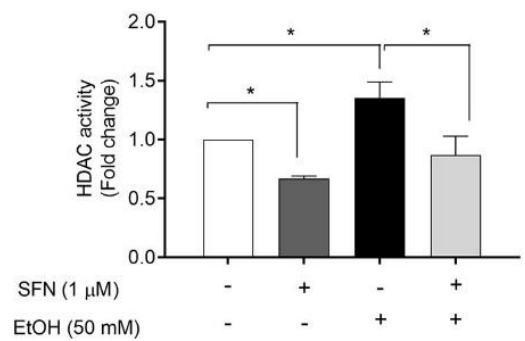

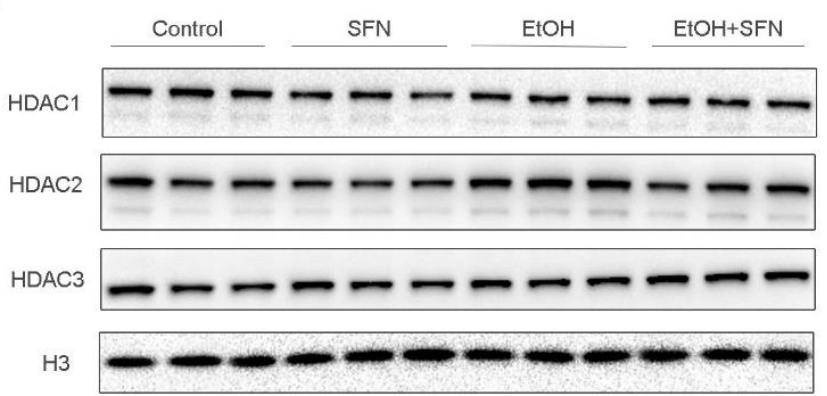
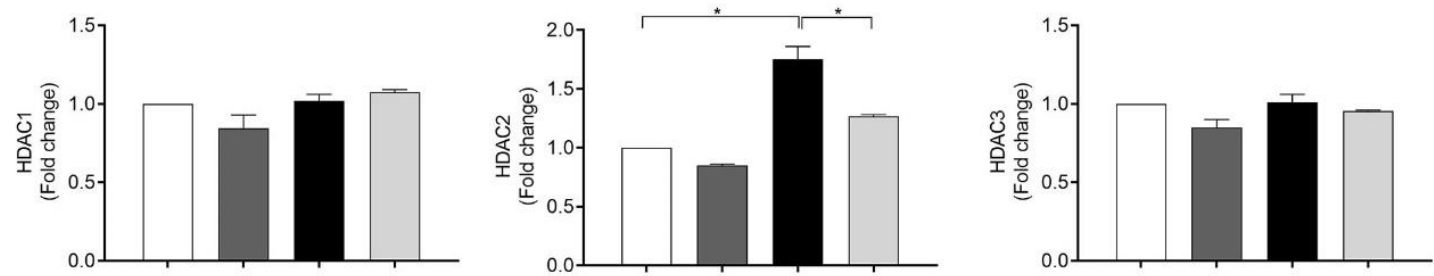

SFN $(1 \mu \mathrm{M})$ EtOH (50 mM)

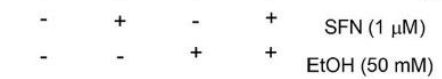

Figure 4. 1. SFN treatment significantly decreased HDAC activity and HDAC2 expression in ethanol-exposed hNCCs. hNCCs were pre-treated with $1 \mu \mathrm{M} \mathrm{SFN}$ for 24 hours and then co-treated with $1 \mu \mathrm{M}$ SFN and $50 \mathrm{mM}$ ethanol for an additional 24 hours. (A) The nuclear protein was isolated from control and treated hNCCs, and the HDAC activity was determined as described in methods. (B) The protein expression of HDAC1, HDAC2, and HDAC3 in control and treated hNCCs was determined by Western blot. Data are expressed as fold change over control and represent the mean \pm SEM of three separated experiments. ${ }^{*} p<0.05$. 


\section{SFN diminished the ethanol-induced reduction of $\mathrm{H} 3$ acetylation at the promoters of the selected anti-apoptotic genes in hNCCs.}

To determine whether ethanol-induced increase in HDAC activity and upregulation of HDAC2 can result in the reduction of $\mathrm{H} 3$ acetylation at the promoters of the selected anti-apoptotic genes and whether SFN that can inhibit HDAC activity and diminish ethanol-induced up-regulation of HDAC2 can prevent ethanol-induced reduction of $\mathrm{H} 3$ acetylation at the promoters of anti-apoptotic genes, the expression of acetylated histone $\mathrm{H} 3$ was determined by western blot. We found that ethanol exposure significantly decreased the levels of acetylated histone $\mathrm{H} 3$ in hNCCs. Co-treatment with SFN diminished the reduction of acetylated histone $\mathrm{H} 3$ in ethanol-exposed hNCCs. We then tested whether ethanol exposure can decrease the acetyl-histone $\mathrm{H} 3$ binding to the promoters of the identified anti-apoptotic genes in hNCCs by using ChIP-qPCR. Exposure to ethanol significantly decreased the binding of acetylated histone $\mathrm{H} 3$ to the promoters of AKT1, BIRC6 and XIAP. Co-treatment with SFN significantly diminished ethanol-induced reduction of the binding of acetylated histone $\mathrm{H} 3$ to the promoters of these anti-apoptotic genes in hNCCs (Figure 4.2B). These results demonstrated that ethanol exposure can reduce the binding of acetylated histone $\mathrm{H} 3$ to the promoters of the selected anti-apoptotic genes in hNCCs, which can be prevented by SFN. 


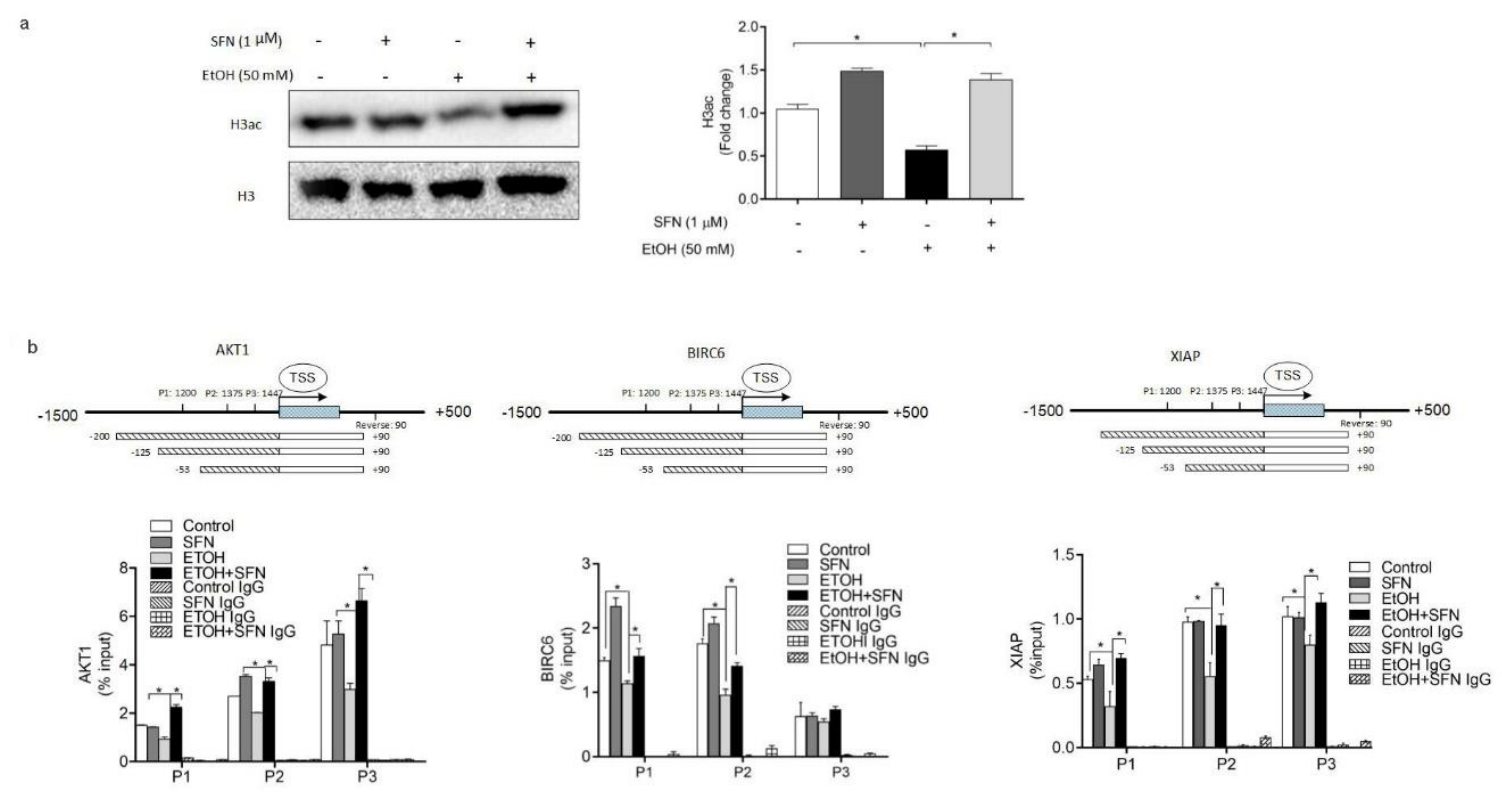

Figure 4. 2. SFN increased acetyl-histone $\mathrm{H} 3$ protein levels and histone $\mathrm{H} 3$ acetylation at the promoters of AKT1, BIRC5, and XIAP in ethanol-exposed hNCCs. hNCCs were pre-treated with $1 \mu \mathrm{M}$ SFN for 24 hours and followed by 24 hours of concurrent exposure of $1 \mu \mathrm{M}$ SFN and $50 \mathrm{mM}$ ethanol. (A) The acetyl-histone $\mathrm{H} 3$ protein expression was determined by using Western blot. (B) The schematic depiction of the promoter regions of each gene and primer sets for chip experiment. Acetyl-histone $\mathrm{H} 3$ enrichment at corresponding sites (P1-P3) was determined by ChIP-qPCR. Data are expressed as fold change over control (A) or percentage of input (B) and represent the mean \pm SEM of three separated experiments. ${ }^{*} p<0.05$. TSS: Transcriptional start site. 
3. SFN treatment diminished ethanol-induced down-regulation of AKT1, BIRC6, and XIAP in ethanol-exposed hNCCs

To determine whether SFN-induced increase in acetylation at the promoters of AKT1, BIRC6 and XIAP leads to the diminished down-regulation of of these genes in ethanol-exposed hNCCs, the mRNA expression of AKT1, BIRC6 and XIAP was determined by using quantitative RT-PCR. The result has shown that ethanol exposure significantly decreased the mRNA expression of AKT1, BIRC6 and XIAP in hNCCs. SFN treatment significantly diminished the ethanolinduced down-regulation of these genes (Figure 4.3). 

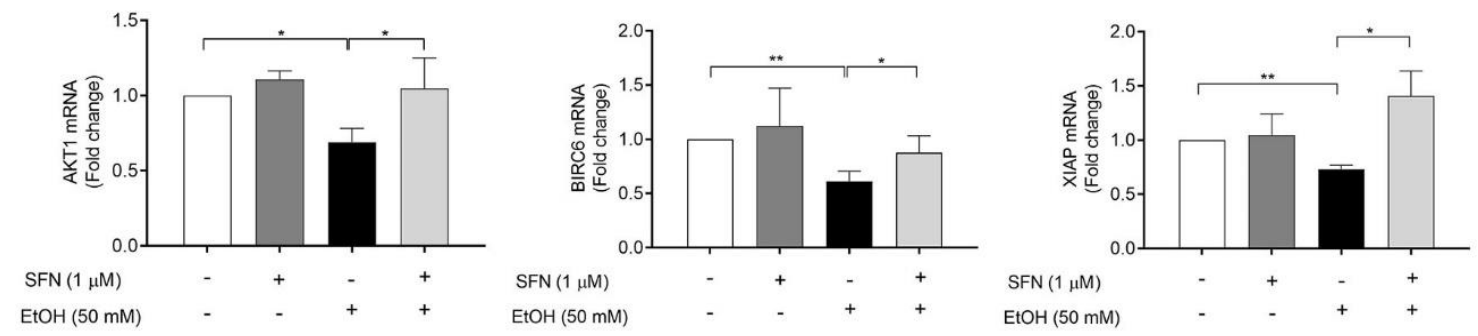

Figure 4. 3. SFN treatment significantly increased the mRNA expression of AKT1, BIRC6 and XIAP in ethanol-exposed hNCCs. hNCCs were pre-treated with $1 \mu \mathrm{M}$ SFN for 24 hours and then co-treated with $1 \mu \mathrm{M} \mathrm{SFN}$ and $50 \mathrm{mM}$ ethanol for an additional 24 hours. The mRNA expression of AKT1, BIRC6 and XIAP was determined by quantitative RT-PCR. Data are expressed as fold change over control and represent the mean \pm SEM of three separated experiments. 


\section{Knockdown of HDAC2 prevented ethanol-induced down-regulation of AKT1, XIAP and BIRC6 in hNCCs.}

To confirm that HDAC2 contributes to the ethanol-induced reduction of the binding of acetylated histone $\mathrm{H} 3$ to the promoters of the selected anti-apoptotic genes and subsequent down-regulation of these genes, HDAC2 was knocked down by siRNA in hNCCs before treatment with ethanol and SFN. We found that ethanol exposure resulted in a significant decrease in mRNA expression of AKT1, BIRC6 and XIAP. Protein expression of AKT1, BIRC6 and XIAP was also decreased in ethanol-exposed hNCCs. The knockdown of HDAC2 significantly diminished ethanol-induced decreases in the mRNA and protein expression of AKT1, BIRC6 and XIAP. The knockdown of HDAC2 also enhanced the effects of SFN on the mRNA and protein expression of AKT1, BIRC6 and XIAP (Figure 4.4A, B). These results demonstrate that knockdown of HADC2 can diminish ethanolinduced repression of anti-apoptotic genes in hNCCs. 

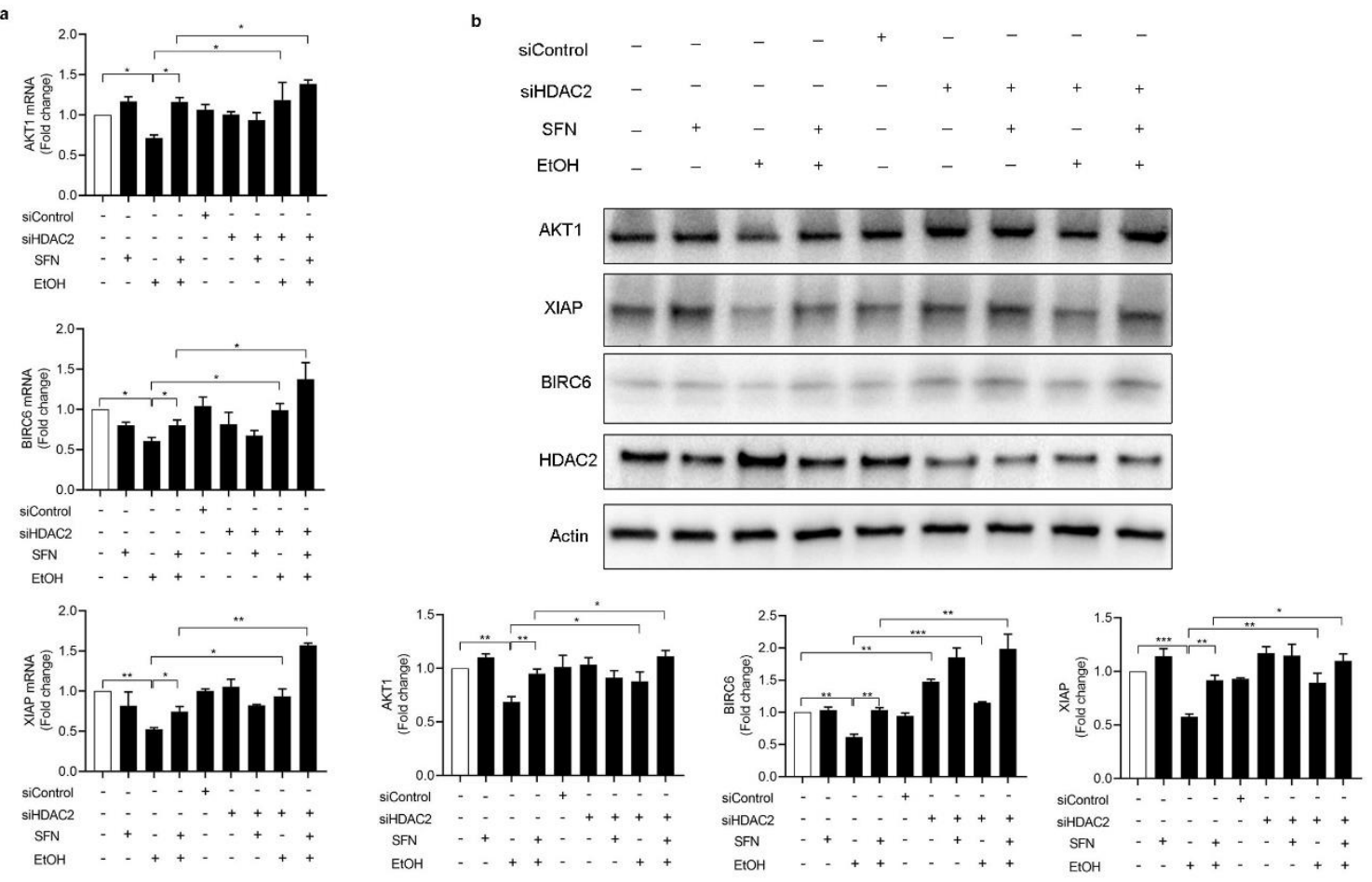

Figure 4. 4. Knockdown of HDAC2 prevented ethanol-induced down-regulation of AKT1, XIAP and BIRC6 in hNCCs. hNCCs transfected with control-siRNA or HDAC2-siRNA were treated with $1 \mu \mathrm{M}$ SFN or $50 \mathrm{mM}$ ethanol or co-treated with SFN and ethanol for 24 hours. (A) The mRNA expressions of AKT1, BIRC5, and XIAP were determined by quantitative RT-PCR. (B) The protein level of AKT1, BIRC5 and XIAP were determined by western blotting. Data are expressed as fold change over control and represent the mean \pm SEM of three separated experiments. ${ }^{*} p<0.05,{ }^{* *} p<0.01,{ }^{* *} p<0.001$. 


\section{The knockdown of HDAC2 and SFN treatment prevented ethanol- induced apoptosis in hNCCs.}

To determine whether ethanol-induced up-regulation of HDAC2 and subsequent reduction of the binding of acetylated histone $\mathrm{H} 3$ to the promoters of the selected anti-apoptotic genes and repression of these genes contribute to ethanol-induced apoptosis and whether SFN can prevent ethanol-induced apoptosis by diminishing ethanol-induced up-regulation of HDAC2, HDAC2 was knocked down by siRNA in hNCCs. We found that ethanol exposure significantly increased apoptosis in hNCCs. The knockdown of HDAC2 by siRNA or downregulation of HDAC2 by SFN significantly diminished ethanol-induced apoptosis in hNCCs (Figure 4.5). These results demonstrate that ethanol-induced up-regulation of HDAC2 contributes to ethanol-induced apoptosis and that SFN can prevent ethanol-induced apoptosis in hNCCs by diminishing ethanol-induced up-regulation of HDAC2. 


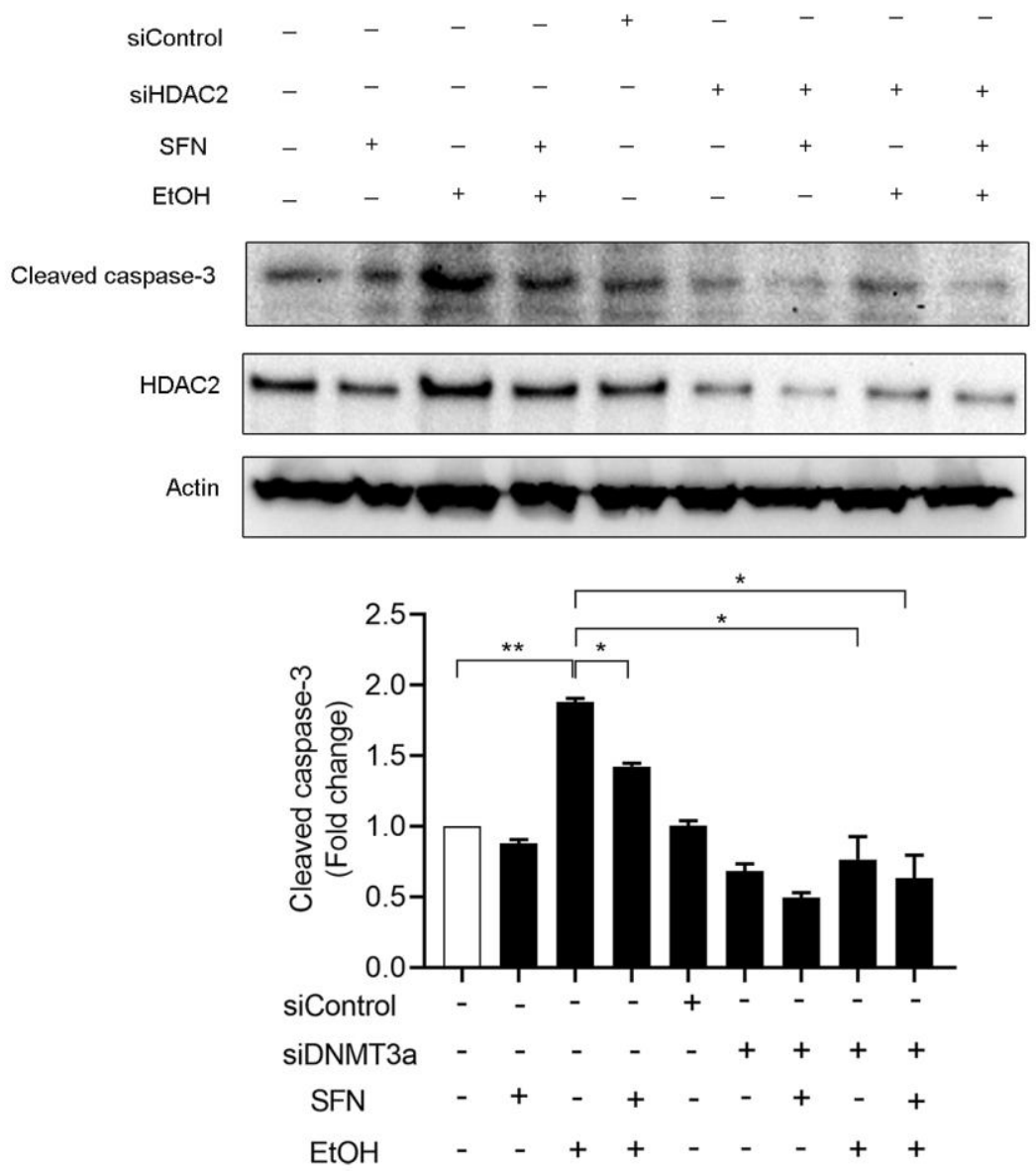

Figure 4. 5. The knockdown of HDAC2 by siRNA or SFN treatment prevented ethanol-induced apoptosis in hNCCs. hNCCs transfected with siRNA-HDAC2 or siRNA-control were treated with $1 \mu \mathrm{M}$ SFN or $50 \mathrm{mM}$ ethanol or co-treated with ethanol and SFN for an additional 24 hours, the protein expression of HDAC2 and cleaved caspase-3 were determined by Western blotting. Data are expressed as fold change over control and represent the mean \pm SEM of three separated experiments. ${ }^{*} p<0.05,{ }^{* *} p<0.01$. 


\section{Discussion}

A recent study has shown that SFN can epigenetically restore the expression of $\mathrm{Bcl} 2$ and attenuating ethanol-induced apoptosis by enhancing the acetylation levels on histone $\mathrm{H} 3$ at the promoter of $\mathrm{Bcl} 2{ }^{18}$. The results from this study have shown that treatment with SFN significantly decreased the enzymatic activity of HDACs in ethanol-exposed hNCCs, which is consistent with the results from our and other's work ${ }^{18,125,175}$. I also found that ethanol exposure decreased the histone $\mathrm{H} 3$ acetylation, suggesting that ethanol-induced inhibition of HDACs activity is associated with a decrease in histone $\mathrm{H} 3$ acetylation levels. This result was supported by the study that has shown that ethanol-induced increase in whole hippocampus HDAC activity was related to a decrease in histone $\mathrm{H} 3$ acetylation levels ${ }^{176}$. I also found that treatment with SFN significantly diminished the ethanolinduced decrease in histone $\mathrm{H} 3$ acetylation. In addition, ethanol exposure significantly reduced the level of histone $\mathrm{H} 3$ acetylation at the promoters of AKT1, BIRC6 and XIAP. Treatment with SFN prevented ethanol's effect on the histone $\mathrm{H} 3$ acetylation at the promoters of AKT1, BIRC6 and XIAP.

To further explore the molecular mechanisms underlying the changes of histone $\mathrm{H} 3$ acetylation at the promoters of anti-apoptotic genes, I have analyzed the protein levels of HDAC1, HDAC2 and HDAC3. These three HDACs belong to the class I HDACs, which are expressed in all tissues and localized mainly in the nucleus. It was reported that class I HDACs were associated with embryonic lethality ${ }^{177}$ and that HDAC2 has been shown to be a crucial molecule to preserve

hippocampal memory and normal cognitive function ${ }^{166}$. The study by Xiang $B$ et 
al., has also demonstrated that HDAC2 was involved in regulating early brain development ${ }^{178}$. Here, I found that ethanol exposure significantly increased HDAC2 expression in hNCCs. This result is consistent with the in vivo study showing that an increase in HDAC2 expression in aged male offspring after maternal consumed ethanol during periconceptional period ${ }^{179}$, as well as an in vitro study demonstrating that ethanol treatment can increase HDAC2 expression in a dose-dependent manner in the human neuronal cell line ${ }^{180}$. Interestingly, the HDAC inhibitor, trichostatin (TSA) can attenuate alcohol-drinking behaviors, with a concomitant reduction in the expression of HDAC2 ${ }^{181}$. HDAC inhibitor also significantly inhibited the ethanol-induced increase of HDAC2 ${ }^{180}$. Consistent with these findings, I have found that SFN, as an HDAC inhibitor, can decrease the ethanol-induced increase in HDAC2 expression and HDAC activity in hNCCs.

Previous studies have shown that HDACs can modulate acetylation within given gene promoters and regulate the transcription of the specific genes. For example, HDAC inhibitor VPA upregulated the expression of anti-apoptotic gene $\mathrm{Hsp} 70$ by enhancing the acetylation of histone $\mathrm{H} 3$ at $\mathrm{Hsp} 70$ promoter $^{68}$. In addition, in ethanol-exposed adulthood rat, high HDAC activity and HDAC2 expression and deficits in histone $\mathrm{H} 3$ acetylation at the promoters of brain-derived neurotrophic factor (BDNF) and decreased expression of BDNF were observed, which were reversed by knockdown of HDAC2 using $\mathrm{TSA}^{176}$. In the present study, I found that knockdown of HDAC2 by transfected with siRNA-HDAC2 significantly increased the expression of AKT1, XIAP and BIRC6 at both mRNA and protein levels, and it can also enhance SFN's effects on promoting the expression of AKT1, XIAP and 
BIRC6 in ethanol-exposed hNCCs. These results suggest that ethanol-induced upregulation of HDAC2 and an increase in HDAC activity contribute to ethanolinduced down-regulation of the anti-apoptotic genes in hNCCs.

Increasing evidence has shown that the downregulation of HDACs can alleviate apoptosis in vitro and in vivo68, 182, 183 . For example, Marisela et al., has reported that HDAC2 inhibitor TSA exerted a neuroprotective effect through reducing ethanol-induced excessive ROS $^{180}$. Furthermore, inhibition of HDAC2 by its inhibitor CAY10683 offers a protective effect on inhibiting apoptosis in mouse model $^{183}$. As an HDAC inhibitor, SFN can also inhibit LPS-induced apoptosis in monocyte-derived dendritic cells, accompanied by a reduction of HDAC activity and the expression of HDAC6 and HDAC10 ${ }^{118}$. Additionally, our recent study has shown that SFN can prevent ethanol-induced apoptosis through inhibiting HDAC activity and increase the level of histone $\mathrm{H} 3$ acetylation at the promoter of $\mathrm{Bcl} 2{ }^{18}$. Consistent with these findings, I have found that knockdown of HDAC2 significantly decreased caspase-3 activation in ethanol-exposed hNCCs and enhanced SFN's protective effects on ethanol-induced apoptosis in hNCCs, suggesting that SFN can diminish hNCCs from ethanol-induced apoptosis by inhibiting HDAC2.

In summary, the present study indicated that treatment with SFN significantly decreased the ethanol-induced increase in the enzymatic activity of HDACs and inhibited ethanol-induced upregulation of HDAC2. Treatment with ethanol significantly decreased acetylation of histone $\mathrm{H} 3$ at the promoter of AKT1, BIRC6 and XIAP, which can be prevented by co-treatment with SFN. Diminished reduction of acetylation at the promoters of AKT1, BIRC6 and XIAP induced by 
SFN reduced ethanol-induced down-regulation of these genes in ethanol-exposed hNCCs. Knockdown of HDAC2 also prevented the ethanol-induced downregulation of AKT1, BIRC6 and XIAP, and subsequently prevented apoptosis in ethanol-exposed hNCCs. These findings demonstrate that SFN can diminish ethanol-induced apoptosis in hNCCs by modulating HDAC2 and diminishing histone acetylation at the promoters of the selected anti-apoptotic genes. 


\section{CHAPTER V}

SULFORPHANE PROTECTS AGAINST ETHANOL-INDUCED APOPTOSIS IN NEURAL CREST CELLS AND ZEBRAFISH EMBRYOS THROUGH MODULATING HISTONE METHYLATION AND THE EXPRESSION OF SNAIL1 AND RESTORING EMT

\section{A. Introduction}

Fetal Alcohol Spectrum Disorder (FASD) is a major developmental defect caused by alcohol consumption by women during pregnancy. FASD is characterized by craniofacial abnormalities, mental retardation and behavior defect $^{184-186}$. Studies have demonstrated that ethanol-induced excessive cell death in the specific cell population is one of the major mechanisms underlying the pathogenesis of FASD ${ }^{187,188}$. Our studies and others have shown that ethanol can induce apoptosis in neural crest cells (NCCs) and that ethanol-induced apoptosis in NCCs contributes significantly to ethanol-induced malformations ${ }^{132,} 155,189$.

NCC is a multipotent and migratory progenitor cell population that originates between the neural plate and non-neural ectoderm ${ }^{10}$. After induction at the border of the neural plate, NCCs leave their original location through a delamination process and migrate ventrally to differentiate into a diversity of neural and nonneural cell types, including neuron, glia, craniofacial cartilage, bone and connect 
tissue $^{91-93,}{ }^{190}$. NCC delamination process involves epithelial-to-mesenchyme transition (EMT). EMT is a process that orchestrates a change from an epithelial to a mesenchymal phenotype, a process that increases migratory properties, invasiveness and apoptotic resistance ${ }^{95,}{ }^{96}$. EMT is essential for both normal development and cancer invasion and metastasis ${ }^{97-99}$. During embryonic development, NCCs undergo an EMT and then dissociate from the neural folds and differentiate to a diversity of cell types ${ }^{10,91,93}$. EMT also plays a pivotal role in promoting tumor proliferation, invasion, and metastasis, exerting an anti-apoptosis effect $^{100,191}$. Studies have shown that EMT conferred resistance to UV-induced apoptosis in three murine mammary epithelial cell lines ${ }^{102}$. Park et al. have also shown that $\alpha$-mangostin can inhibit EMT and induce apoptosis in an osteosarcoma cell line ${ }^{103}$. However, the roles of EMT in ethanol-induced apoptosis in NCCs and in the pathogenesis of FASD remain to be defined.

One of the well-known transcriptional factors that regulate EMT is Snail1 ${ }^{104-106}$. Snail1 promotes EMT primarily through the directly repressing E-cadherin, an EMT suppressing factor ${ }^{104,107,192}$. The expression of Snail1 can be regulated by many mechanisms, including epigenetic regulation. Epigenetic modification generally includes DNA methylation and histone modification, which includes histone acetylation and methylation ${ }^{48}$. Among the histone methylation, the triple methyl modification on the fourth lysine of histone 3 (H3K4me3) typically facilitates the activation of gene transcription ${ }^{72}$, while lysine demethylase $5 A(K D M 5 A)$, the member of KDM superfamily, has been shown to specifically catalyzing the removal of tri-methyl from $\mathrm{H} 3 \mathrm{~K} 4$ and initiating the transcriptional repression 
process $^{193-195}$. Previous studies have established that KDM5A can cooperate with different transcriptional factors to selectively repress gene transcription ${ }^{193,}{ }^{195-197}$. Given the intimate association between $\mathrm{H} 3 \mathrm{~K} 4$ methylation and neurodevelopmental disorder, it is rational to assume that KDMs that are responsible for demethylation of $\mathrm{H} 3 \mathrm{~K} 4 \mathrm{me}$ associated with neurodevelopmental disorder ${ }^{194,198,199 .}$

Sulforaphane (SFN) is a vegetable-derived isothiocyanate that is abundant in cruciferous vegetables such as broccoli. Our previous studies have shown that SFN exerts an anti-apoptotic effect through upregulating antioxidant gene Nrf-2 in NCCs ${ }^{17}$. Our recent studies have also shown that SFN can prevent ethanolinduced apoptosis in NCCs by reversing ethanol-induced reduction of histone acetylation at the promoter of the anti-apoptotic gene, $\mathrm{Bcl} 2^{18}$. More recently, SFN had been reported to act as an inhibitor of histone deacetylase (HDAC) and DNA methyltransferase (DNMT), two key enzymes involved in histone deacetylation and DNA methylation, respectively, to cause epigenetic modification of genes in varied types of cells, including the genes involved in EMT in cancer cells ${ }^{115-117}$. Interestingly, studies have also shown that HDAC inhibitor also has a high inhibitory effect on $\mathrm{KDMs}^{200}$ and that HDAC inhibitor decreased KDM5A expression and modulated the expression of histone markers $\mathrm{H} 3 \mathrm{Ac}$ and $\mathrm{H} 3 \mathrm{~K} 4 \mathrm{me} 3^{201}$. However, the role of SFN and KDMs in the epigenetic regulation of EMT transcriptional factors and ethanol-induced apoptosis in NCCs remains unknown. 
In the present study, I tested the hypothesis that SFN can protect against ethanol-induced apoptosis by restoring EMT through epigenetically modulating the expression of Snail1 in NCCs. I found that treatment with SFN significantly reversed ethanol-induced changes in the expression of E-cadherin, and restored EMT in NCCs. I also found that ethanol exposure significantly reduced the levels of H3K4me3 at the promoters of Snail1. SFN treatment reduced the ethanolinduced reduction of $\mathrm{H} 3 \mathrm{~K} 4 \mathrm{me} 3$ at the promoter regions of the Snail1 gene and restored Snail1 gene expression and EMT. In addition, I found that ethanol exposure increased the expression of KDM5A, which was decreased by cotreatment with SFN. Knockdown KDM5A by siRNA prevented the ethanol-induced decrease of H3K4me3 enrichment at Snail1 promoter, restored Snail1 gene expression and subsequently restored EMT in NCCs exposed to ethanol. I have also found siRNA mediated downregulation of KDM5A dramatically diminished ethanol-induced apoptosis in NCCs as well as zebrafish embryos. These results demonstrate that ethanol exposure can induce apoptosis in NCCs by inhibiting EMT and that SFN can protect against ethanol-induced apoptosis by epigenetically regulating the expression of Snail1 and restoring EMT.

\section{B. Experimental procedures}

\section{Cell culture and treatment}

NCCs (JoMa 1.3 cell) were cultured on culture dishes coated with fibronectin. NCCs were treated with SFN and ethanol, as described in Chapter II, Section A. 


\section{Quantitative real-time PCR}

The mRNA expression of Snail1, KDM5A, E-cadherin, Vimentin, and Snail1 were measured by quantitative real-time RT-PCR as described in Chapter II, Section C. 2.

\section{Western blot analysis}

The protein level of KDM5A, H3K4me3, H3, E-cadherin, Vimentin, Cleaved caspase-3, and Snail1 were determined by western blotting as described in Chapter II, Section C. 3.

\section{Chip-qRT-PCR analysis}

ChIP experiments were performed as described in Chapter II, C. 4. Chromatin was immunoprecipitated with antibodies to H3K4me3. The qRT-PCR was carried out with primers shown in Chapter II, C. 4.

\section{TUNEL staining}

Analysis of apoptosis in $24 \mathrm{hpf}$ zebrafish embryos of control and experimental groups was performed by using TUNEL staining as described in Chapter II, B. 3.

\section{Flow cytometry}

Apoptosis in NCCs was determined by using Flow cytometry, as described in Chapter II, C. 5.

\section{7. siRNA Knockdown of KDM5A and Snail1}


For Snail1 or KDM5A siRNA transfection, NCCs were transfected with Snail1 siRNA, KDM5A siRNA or scrambled control siRNA as described in Chapter II, C. 7 .

\section{Statistical analysis}

Statistical analysis was performed, as described in Chapter II, C. 8.

\section{Results}

\section{Ethanol exposure induced apoptosis in NCCs}

To determine whether ethanol exposure can induce apoptosis in NCCs, NCCs were exposed to 50 or $100 \mathrm{mM}$ ethanol, and ethanol-induced apoptosis was determined by the analysis of caspase- 3 activation and Annexin $V$ staining. As shown in Figure 5.1A, ethanol exposure resulted in significant increases in caspase-3 activation in a dose-dependent manner, indicating that ethanol treatment can induce apoptosis in NCCs. This result was confirmed by the results from the flow cytometric analysis of Annexin $V$ staining, which have shown that exposure of NCCs to 50 or $100 \mathrm{mM}$ ethanol caused a substantial increase in the number of early apoptotic NCCs (Figure 5.1B) 
a

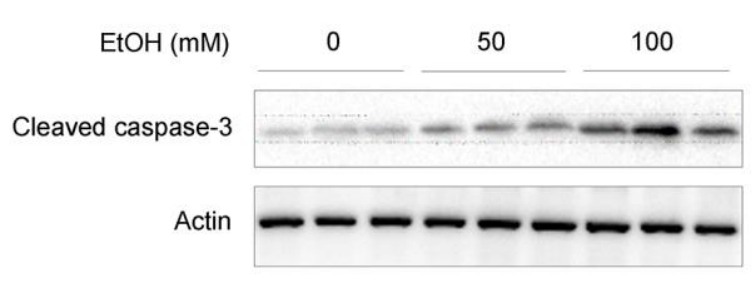

b
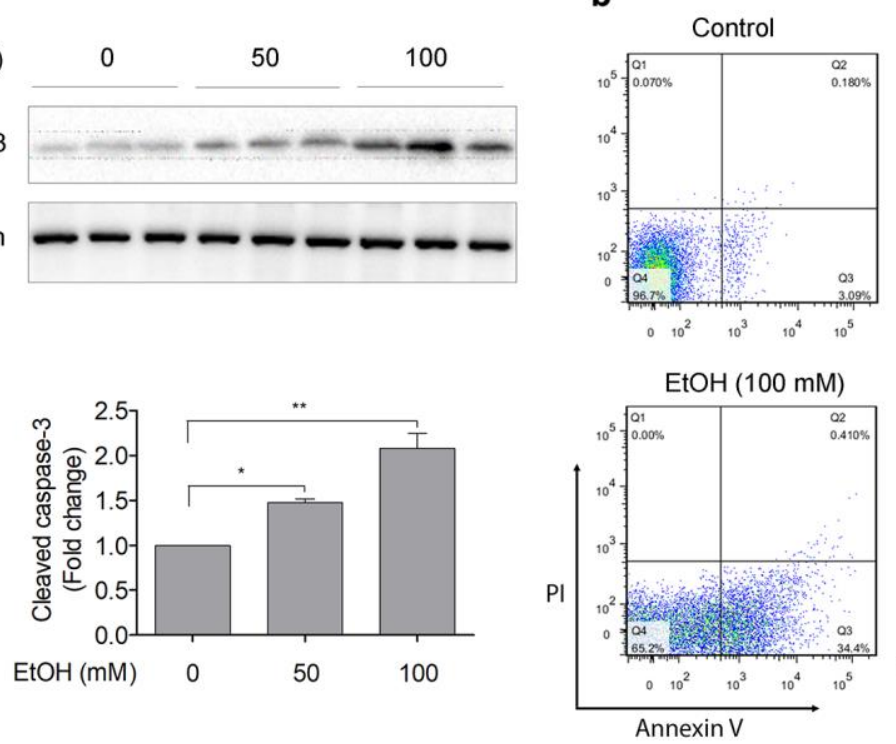
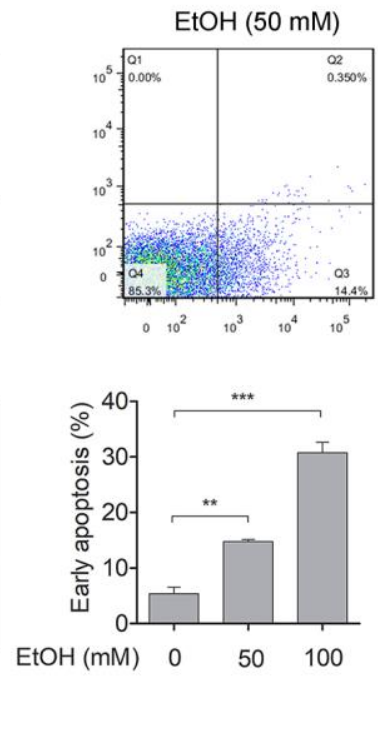

Figure 5. 1. Ethanol exposure induced apoptosis in NCCs. NCCs were exposed to 50 or $100 \mathrm{mM}$ ethanol for $24 \mathrm{~h}$. Apoptosis was determined by the analysis of caspase-3 cleavage by western blotting (A, B) or flow cytometry with Annexin VFITC apoptosis detection kit (B). Data are expressed as fold change over control (A) or percentage of all cells $(B)$ and represent the mean \pm SEM of three separated experiments. ${ }^{*} p<0.05,{ }^{* *} p<0.01$. 


\section{SFN diminished ethanol-induced inhibition of EMT in NCCs}

To determine whether ethanol exposure can induce apoptosis in NCCs by inhibiting EMT and whether SFN can protect NCCs against apoptosis through restoring EMT, NCCs were cultured with $1 \mu \mathrm{M}$ SFN alone for 24 hours, followed by 24 hours of concurrent exposure to $1 \mu \mathrm{M}$ SFN and $50 \mathrm{mM}$ ethanol. As shown in Figure 5. 2, ethanol treatment significantly inhibited EMT in NCCs, as indicated by the increased expression of E-cadherin, an EMT-suppressing marker, and a decreased expression of vimentin, an EMT-promoting marker. Treatment with SFN significantly reversed ethanol-induced changes in the expression of E-cadherin and vimentin and restored EMT in NCCs. These results indicate that ethanol exposure can inhibit EMT in NCCs, which can be reversed by SFN. 

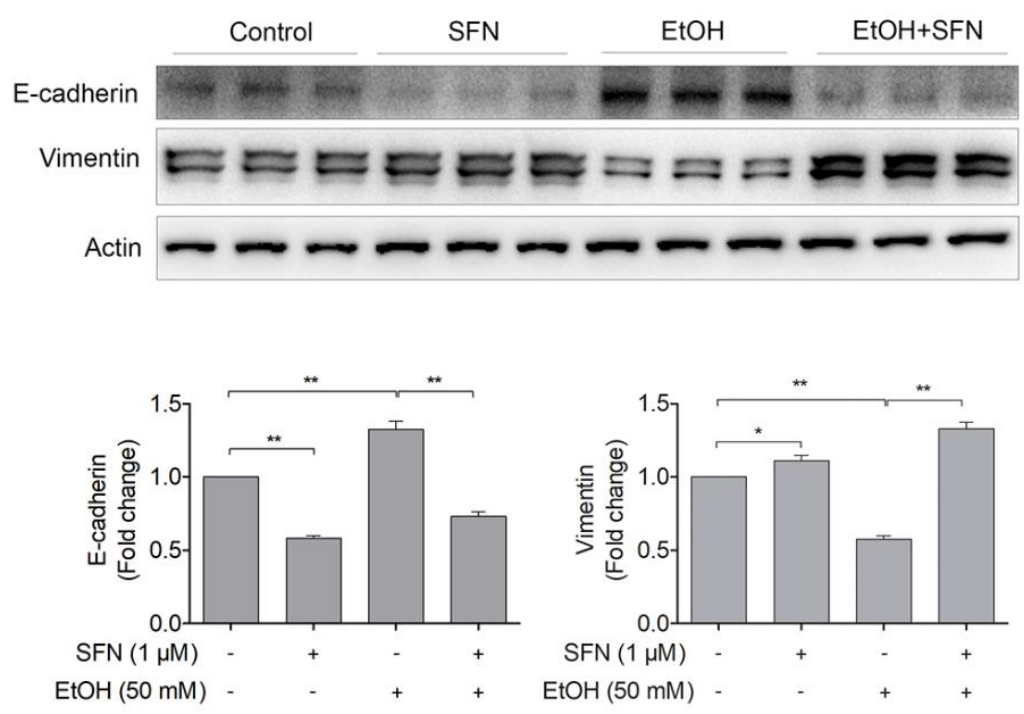

Figure 5. 2. SFN diminished ethanol-induced EMT inhibition in NCCs. NCCs were pre-treated with $1 \mu \mathrm{M} \mathrm{SFN}$ for $24 \mathrm{~h}$ and followed by $24 \mathrm{~h}$ of concurrent exposure to SFN and ethanol. The expression of EMT-suppressing factor E-cadherin and EMT-promoting factor vimentin was determined by western blotting. Data are expressed as fold change over control and represent the mean \pm SEM of three separated experiments. ${ }^{*} p<0.05,{ }^{* *} p<0.01$. 


\section{SFN diminished the ethanol-induced increases in the mRNA and protein expression of KDM5A in NCCs.}

KDM5A, a histone demethylase, has been shown to directly remove trilysine of histone $\mathrm{H}^{193}$, 194, 202. To determine whether KDM5A is involved in the ethanol-induced alteration in histone methylation, the expression of KDM5A was determined in control and ethanol-exposed NCCs. As shown in Figure 5.3A, B, qRT-PCR and Western blot analysis revealed a significant increase in the mRNA and protein expression of KDM5A in ethanol-exposed NCCs. Co-treatment with SFN and ethanol diminished the ethanol-induced up-regulation of KDM5A in NCCs. 
a

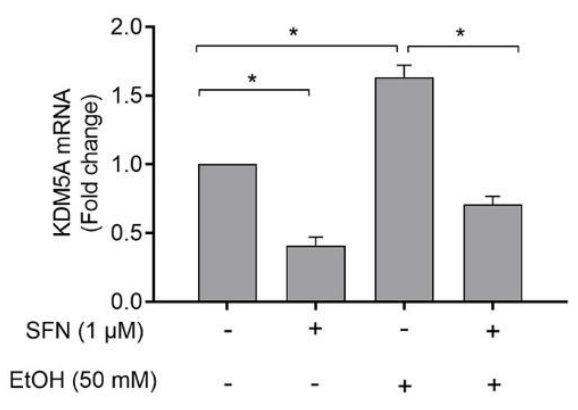

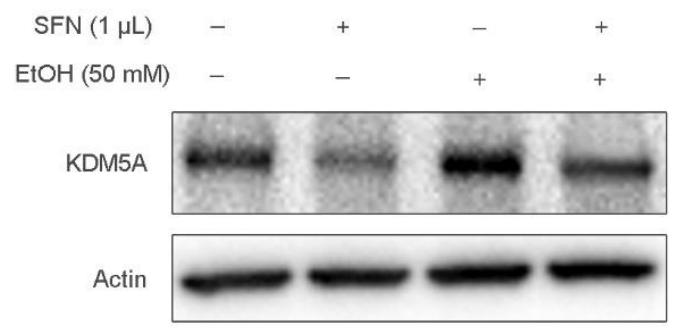

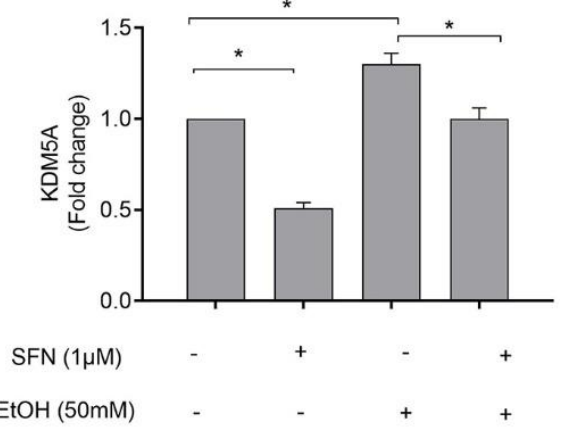

Figure 5. 3. SFN diminished ethanol-induced increases in the mRNA and protein expression of KDM5A in NCCs. NCCs were pretreated with $1 \mu \mathrm{M} \mathrm{SFN}$ for $24 \mathrm{~h}$ and followed by $24 \mathrm{~h}$ of concurrent exposure to SFN and $50 \mathrm{mM}$ ethanol for $24 \mathrm{~h}$. (A) The mRNA expression of KDM5A was determined by quantitative RT-PCR. (B) Protein expression of KDM5A was determined by western blotting. Data are expressed as fold change over control and represent the mean \pm SEM of three separated experiments. ${ }^{*} p<0.05,{ }^{* *} p<0.01$. 


\section{SFN treatment diminished the ethanol-induced reduction in the}

H3K4me3 enrichment at the promoter regions of the Snail1 gene in NCCs.

Snail1 is a key transcriptional repressor of E-cadherin and plays an important role in the regulation of $\mathrm{EMT}^{104,107,192}$. To determine the mechanisms by which ethanol inhibits EMT and SFN diminishes the ethanol-induced inhibition of EMT in NCCs, I next determined whether ethanol and SFN can modulate EMT through epigenetically regulating the expression of Snail1. I first determined whether ethanol exposure can reduce the H3K4 trimethylation (H3K4me3), an epigenetic modification that is associated with the activation of transcription of genes $^{72,203}$. As shown in Figure 5.4A, exposure to $50 \mathrm{mM}$ ethanol resulted in a significant decrease in the levels of $\mathrm{H} 3 \mathrm{~K} 4 \mathrm{me} 3$ in NCCs. Co-treatment with SFN and ethanol significantly increased the $\mathrm{H} 3 \mathrm{~K} 4 \mathrm{me} 3$ expression in NCCs, indicating that ethanol-induced reduction of H3K4 trimethylation in NCCs can be diminished by SFN. In addition, the ChIP-qPCR analysis revealed that ethanol exposure resulted in a significant reduction of $\mathrm{H} 3 \mathrm{~K} 4 \mathrm{me} 3$ enrichment at the promoter regions of Snail1. SFN can diminish the ethanol-induced reduction of $\mathrm{H} 3 \mathrm{~K} 4 \mathrm{me} 3$ enrichment at the promoter regions of Snail1 (Figure 5.4C). 
a
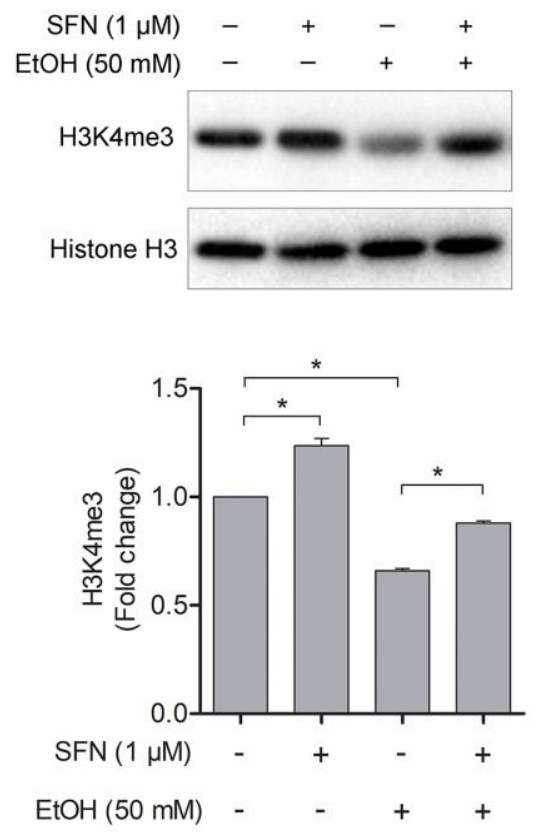

b

Snail1 promoter region

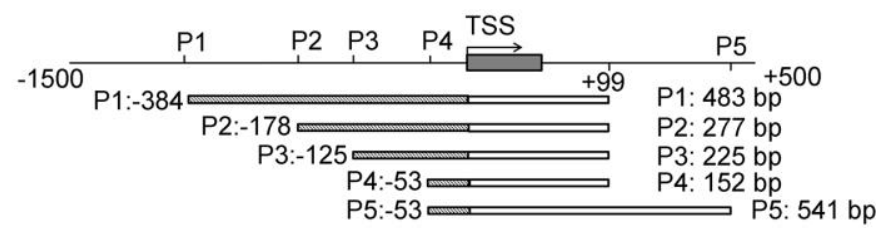

C

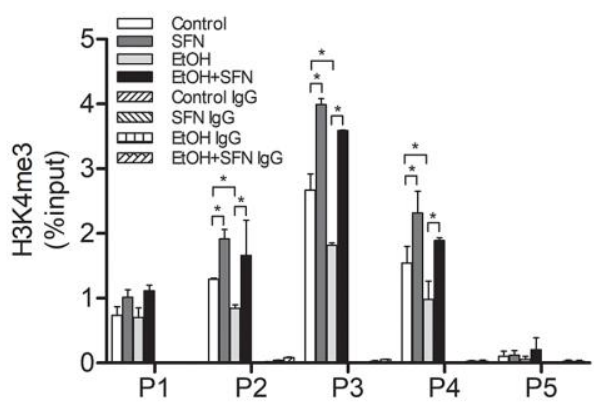

Figure 5. 4. SFN diminished the ethanol-induced reduction of the levels of H3K4me3 at the Snail 1 promoter. (A) The levels of H3K4me3 in control and treated NCCs were determined by western blot. (B) Schematic depiction of the Snail1 promoter and the primer sets for ChIP-qPCR analyses. (C) H3K4me3 enrichment at corresponding sites (P1-P5) was determined by ChIP-qPCR. Data are expressed as fold change over control $(A)$ or the percentage of input $(C)$ and represent the mean \pm SEM of three separated experiments ${ }^{*} p<0.05$. TSS: Transcriptional start site. 


\section{SFN treatment restored the expression of Snail1 in ethanol-exposed NCCs}

I next tested whether the ethanol-induced reduction of $\mathrm{H} 3 \mathrm{~K} 4 \mathrm{me} 3$ enrichment at the promoter regions of Snail1 can downregulate the Snail1 and whether SFN treatment can restore the expression of Snai1 in ethanol-exposed NCCs. As expected, qRT-PCR and Western blot analysis revealed a significant decrease in the mRNA and protein expression of Snail1 in ethanol-exposed NCCs. Treatment with SFN restored the mRNA and protein expression of Snail1 in NCCs exposed to ethanol (Figure 5.5). This result demonstrates that SFN can prevent ethanol-induced down-regulation of Snail1 in NCCs. 

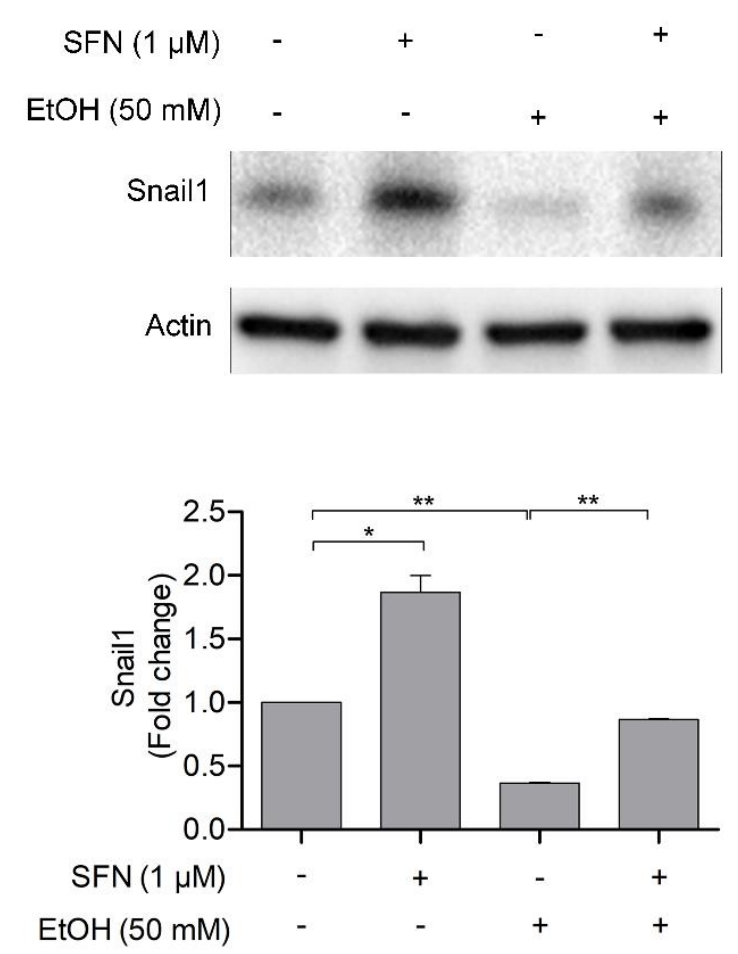

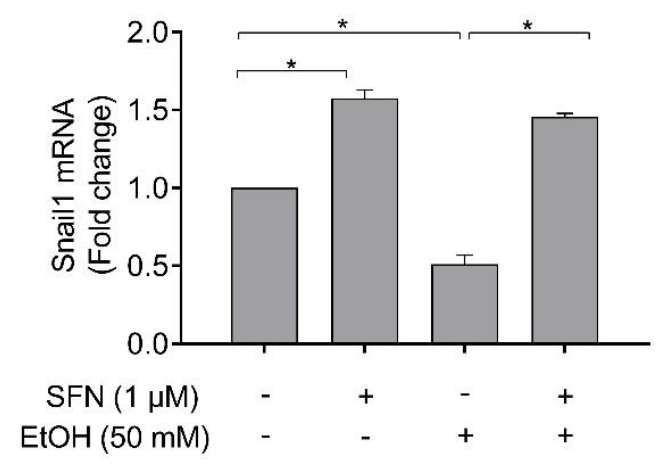

Figure 5. 5. SFN restored Snail1 expression in ethanol-exposed NCCs. NCCs were exposed to $1 \mu \mathrm{M}$ SFN for $24 \mathrm{~h}$, followed by concurrent exposure to $1 \mu \mathrm{M}$ SFN and $50 \mathrm{mM}$ ethanol for $24 \mathrm{~h}$. The expression of Snail1 mRNA (A) and protein (B) was determined by quantitative RT-qPCR and Western blot, respectively. Data are expressed as fold change over control and represent the mean \pm SEM of three separated experiments. ${ }^{*} p<0.05,{ }^{* *} p<0.01$. 


\section{Treatment with SFN significantly decreased the up-regulation of Snail1 target gene E-cadherin in NCCs exposed to ethanol}

To determine whether ethanol-induced down-regulation of Snail1 can increase the expression of E-cadherin, a Snail1 target gene and an EMT suppressing marker, in NCCs and whether SFN can diminish ethanol-induced upregulation of E-cadherin, the mRNA expression of E-cadherin was determined in control and treated NCCs. As shown in Figure 5.6, exposure of NCCs to ethanol resulted in a significant increase in E-cadherin expression. Treatment with SFN significantly diminished ethanol-induced up-regulation of E-cadherin in NCCs. Since the down-regulation of E-cadherin is considered to be a hallmark of EMT ${ }^{106}$, ${ }^{204}$, these results demonstrate that SFN can prevent ethanol-induced inhibition of EMT in NCCs. 


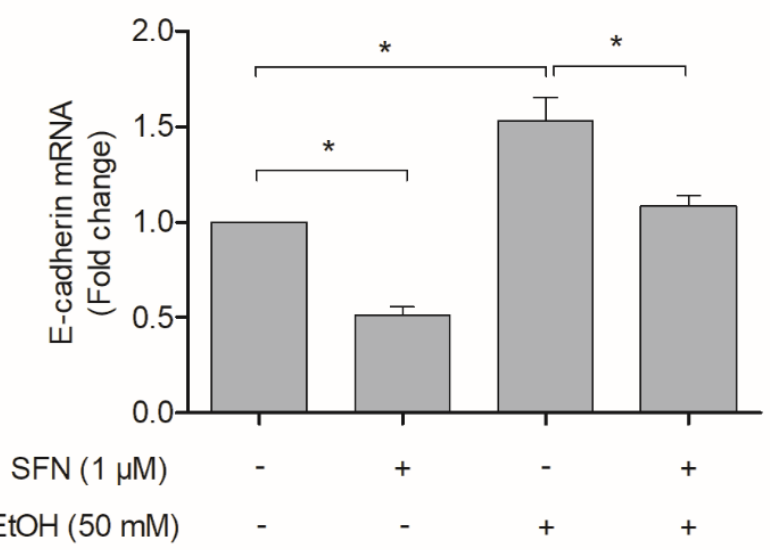

Figure 5. 6. SFN diminished the expression of Snail1 target gene E-cadherin in ethanol-exposed NCCs. NCCs were pre-treated with $1 \mu \mathrm{M} \mathrm{SFN}$ for $24 \mathrm{~h}$ and then exposed to $1 \mu \mathrm{M}$ SFN and $50 \mathrm{mM}$ ethanol for an additional $24 \mathrm{~h}$. The expression of E-cadherin mRNA was determined by quantitative RT-PCR. Data are expressed as fold change over control and represent the mean \pm SEM of three separated experiments. ${ }^{*} p<0.05$. 


\section{SFN treatment significantly diminished ethanol-induced apoptosis in NCCs through up-regulation of Snail1}

To determine whether SFN can prevent ethanol-induced apoptosis, caspase-3 activation and Annexin V staining were examined in control and treated NCCs. As shown in Figure 5.7A, exposure of NCCs to ethanol resulted in a significant increase in caspase-3 activation, indicating that ethanol exposure induced apoptosis in NCCs. Treatment with SFN significantly reduced caspase-3 activation in NCCs exposed to ethanol. To further confirm that SFN can diminish ethanol-induced apoptosis through up-regulation of Snail1, apoptosis was analyzed by the flow cytometric analysis of Annexin V staining in NCCs transfected with control or Snail1 siRNA. I found that knockdown of Snail1 by siRNA significantly increased ethanol-induced apoptosis as compared to the NCCs transfected with control siRNA, confirming that down-regulation of Snail1 can induce apoptosis in NCCs. Down-regulation of Snail1 by siRNA also significantly diminished the protective effects of SFN on ethanol-induced apoptosis in NCCs (Figure 5.7B), indicating that SFN can attenuate ethanol-induced apoptosis by modulating the expression of Snail1, further supporting our hypothesis. 
a

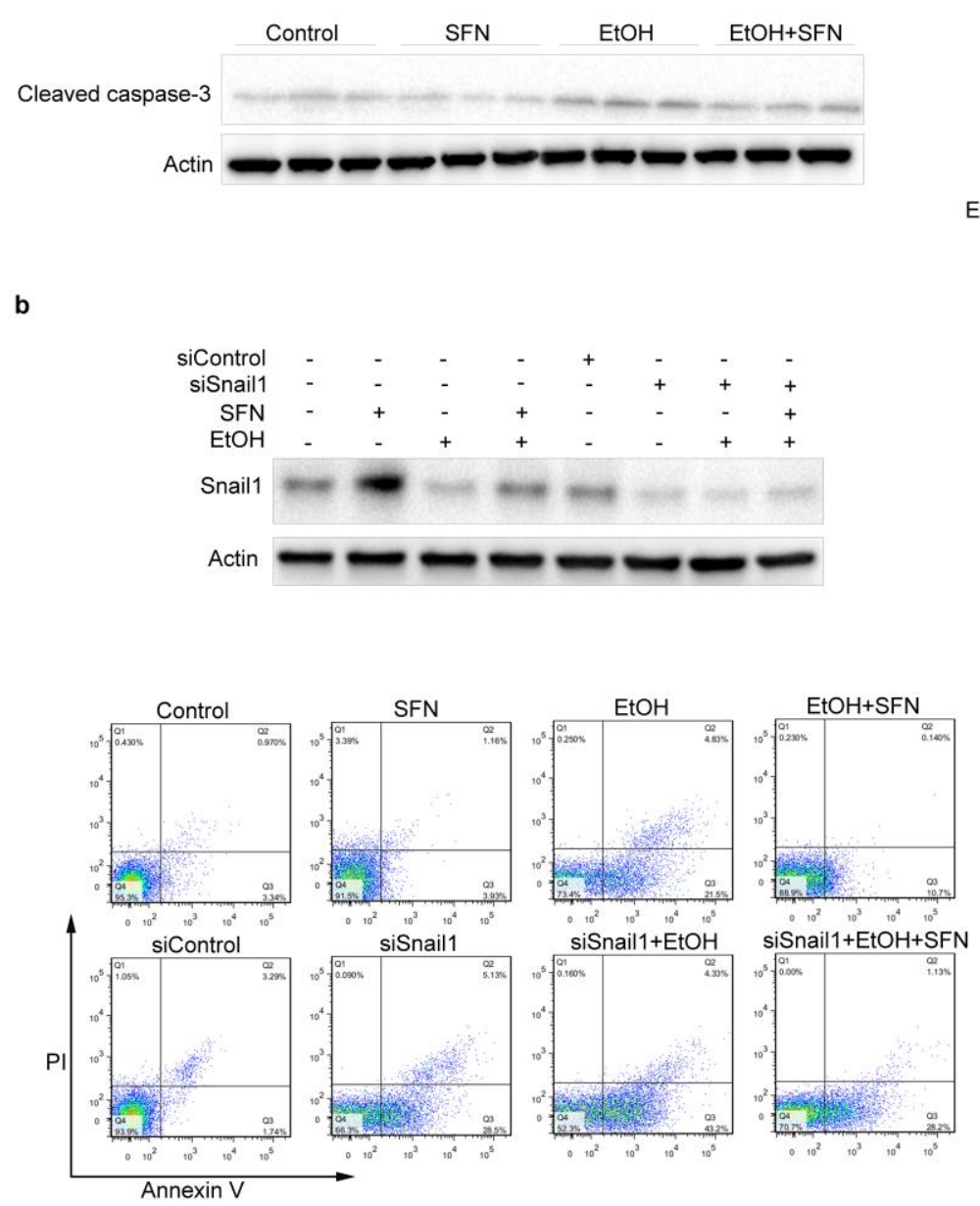

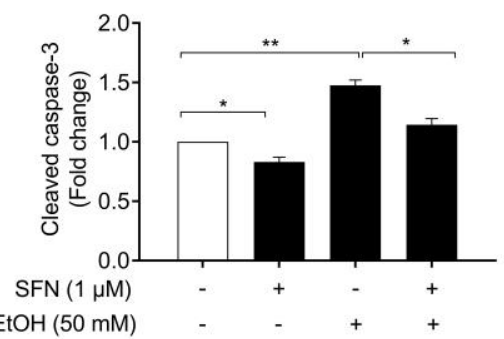
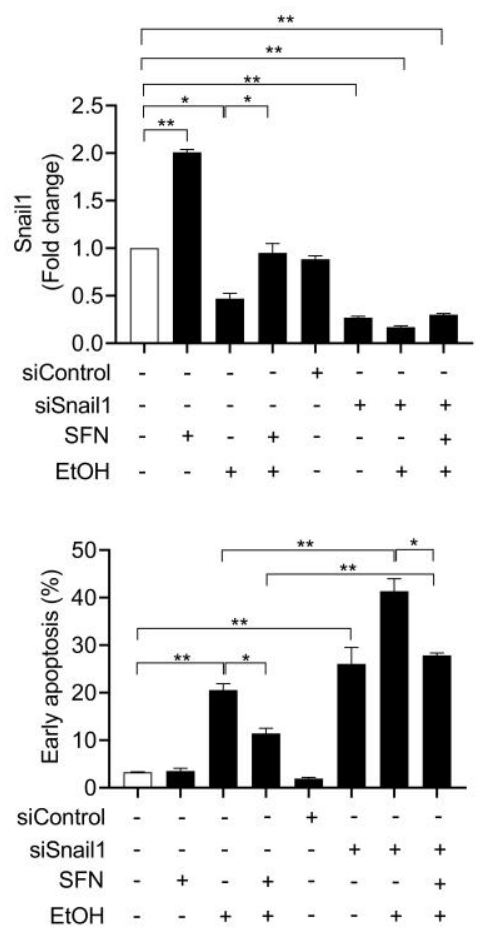

Figure 5. 7. SFN diminished ethanol-induced apoptosis in NCCs through the upregulation of Snail1. (A) NCCs were exposed to $1 \mu \mathrm{M}$ SFN for $24 \mathrm{~h}$, followed by concurrent exposure to $1 \mu \mathrm{M}$ SFN and $50 \mathrm{mM}$ ethanol for $24 \mathrm{~h}$. Apoptosis was determined by the analysis of caspase-3 cleavage using Western blot (B) NCCs transfected with control or Snail1 siRNA was determined by Western blot. Apoptosis was determined by flow cytometry with Annexin V-FITC apoptosis detection kit. Data are expressed as fold change over control $(A)$ or percentage of all cells $(B)$ and represent the mean \pm SEM of three separated experiments. ${ }^{*} p<0.05,{ }^{* *} p<0.01$. 
8. The knockdown of KDM5A significantly diminished the ethanolinduced inhibition of H3K4me3 expression and reduction of H3K4me3 enrichment at Snail1 promoters.

To further explore the effect of KDM5A on the ethanol-induced reduction of $\mathrm{H} 3 \mathrm{~K} 4 \mathrm{me} 3$ enrichment at Snail1 promoter, the expression of H3K4me3 and the H3K4me3 enrichment at Snail1 promoter in NCCs transfected with control or KDM5A siRNA were analyzed. The results indicated that knockdown of KDM5A by siRNA significantly reduced the ethanol-induced inhibition of $\mathrm{H} 3 \mathrm{~K} 4 \mathrm{me} 3$ expression in NCCs (Figure 5.9A). In addition, the ChIP-qPCR analysis revealed that NCCs transfected with siKDM5A significantly diminished ethanol-induced reduction of H3K4me3 at Snail1 promoter regions (Figure 5.9B). 
a
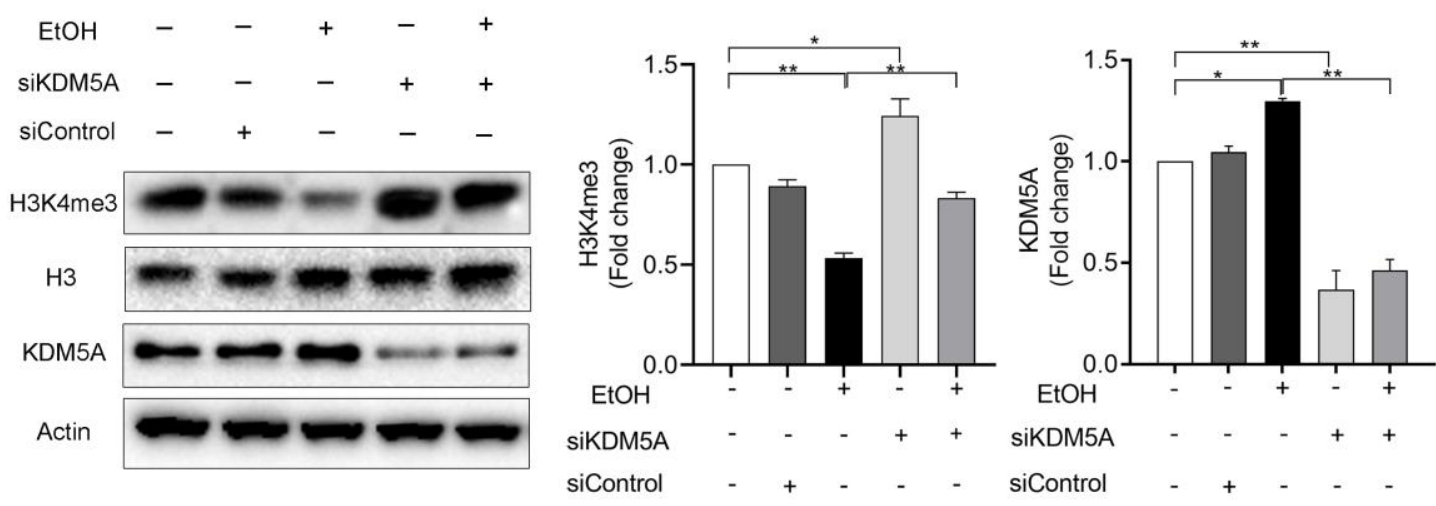

b

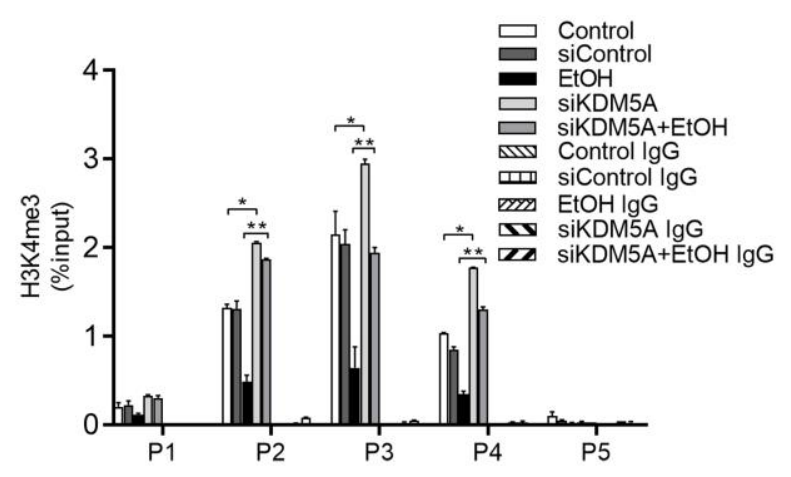

Figure 5. 8. Down-regulation of KDM5A significantly diminished ethanol-induced inhibition of $\mathrm{H} 3 \mathrm{~K} 4 \mathrm{me} 3$ expression and reduction of $\mathrm{H} 3 \mathrm{~K} 4 \mathrm{me} 3$ enrichment at the Snail1 promoter regions in NCCs. NCCs transfected with control siRNA, or KDM5A-siRNA were treated with or without $50 \mathrm{mM}$ ethanol for 24 hours. (A). The nuclear protein was extracted and the expression of $\mathrm{H} 3 \mathrm{~K} 4 \mathrm{me} 3$ and histone $\mathrm{H} 3$ were determined by western blotting. The KDM5A expression at the protein level was determined by western blotting. (B). H3K4me3 enrichment at corresponding sites (P1-P5) was determined by ChIP-qPCR. Data as fold change over control (A) of percentage of input $(B)$ and represent the mean \pm SEM of three separated experiments. ${ }^{*} p<0.05,{ }^{* *} p<0.01$. 


\section{Down-regulation of KDM5A diminished ethanol-induced inhibition of EMT in NCCs.}

To determine whether ethanol-induced up-regulation of KDM5A contributes to ethanol-induced inhibition of EMT, the effects of down-regulation of KDM5A on the expression of Snail1 were determined in ethanol-exposed NCCs. As expected, knockdown of KDM5A prevented the ethanol-induced down-regulation of Snail1 (Figure 5.9A). Snail1 is a key transcriptional factor that promotes EMT by targeting EMT repressing maker E-cadherin ${ }^{96,104,106,107}$. I further determine the mRNA expression of E-cadherin in NCCs transfected with control or KDM5A siRNA. As observed in Figure 5.9B, NCCs transfected with siKDM5A significantly reduced ethanol-induced increase of E-cadherin expression. To further determine whether the downregulation of KDM5A can restore ethanol-induced inhibition of EMT in NCCs, both EMT promoting marker E-cadherin and repressing marker vimentin were analyzed in NCCs transfected with control or KDM5A siRNA. As shown in Figure 5.9C, ethanol treatment significantly inhibits EMT in NCCs, as indicated by the increased protein expression of E-cadherin and a decreased protein expression vimentin. Downregulation of KDM5A significantly reduced ethanolinduced changes in the expression of E-cadherin and vimentin. These results clearly demonstrated that knockdown of KDM5A significantly prevented ethanolinduced inhibition of EMT in NCCs. 

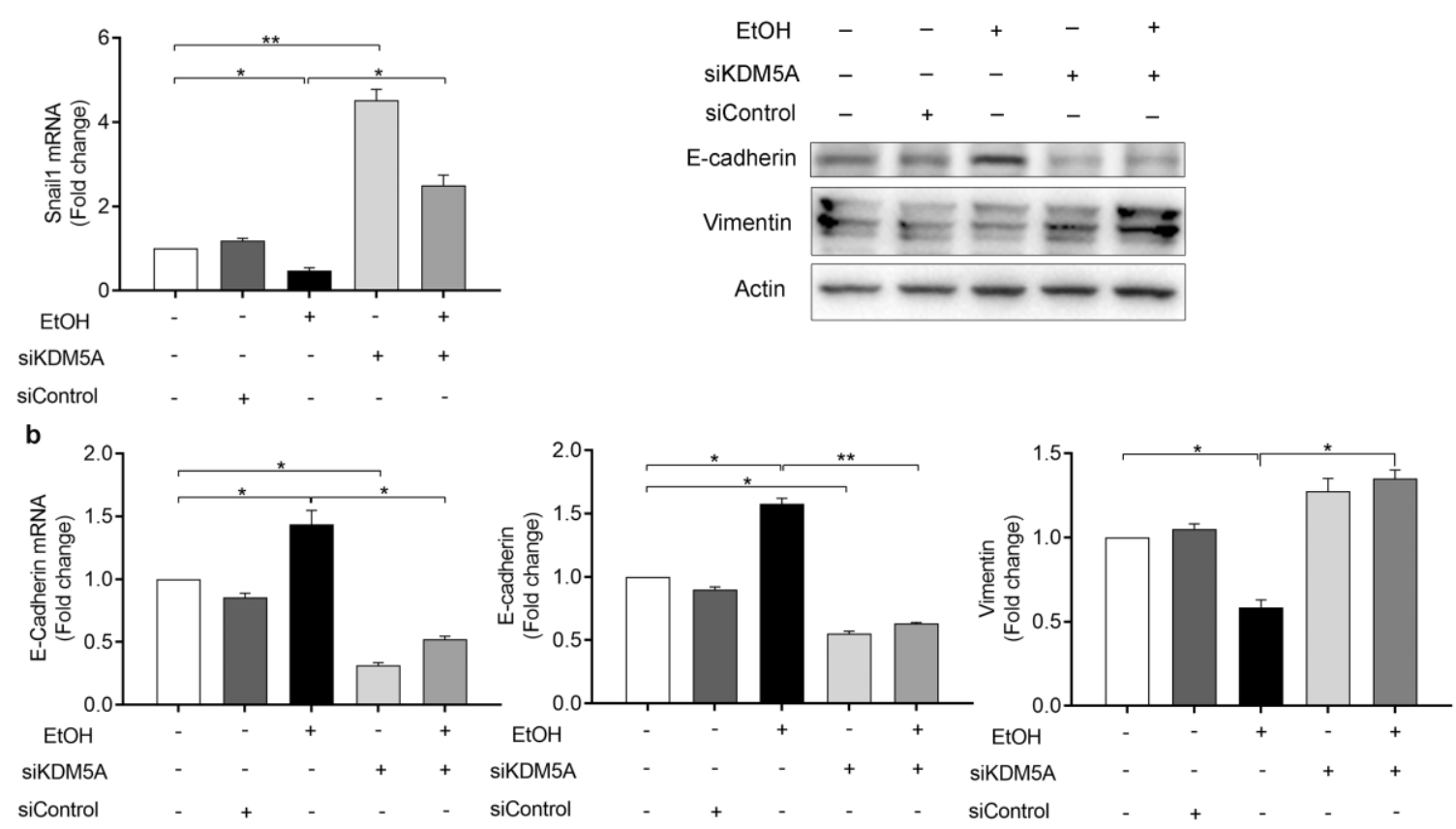

Figure 5. 9. Down-regulation of KDM5A diminished ethanol-induced inhibition of EMT in NCCs. NCCs transfected with control-siRNA or KDM5A-siRNA were treated with or without $50 \mathrm{mM}$ ethanol for 24 hours. The mRNA expression of Snail1 (A) and E-cadherin (B) were determined by quantitative RT-qPCR. The protein expression of E-cadherin and vimentin were determined by western blotting (C). Data are expressed as fold change over control and represent the mean \pm SEM of three separated experiments. ${ }^{*} p<0.05,{ }^{* *} p<0.01$. 


\section{The knockdown of KDM5A significantly decreased ethanol-induced apoptosis in NCCs and zebrafish embryos.}

To determine whether the downregulation of KDM5A can prevent ethanolinduced apoptosis, apoptosis was analyzed by caspase-3 activation and Annexin V staining in control and ethanol-exposed NCCs transfected with control or KDM5A siRNA. As shown in Figure 5.10A, exposure of NCCs to ethanol resulted in a significant increase in caspase-3 activation, knockdown of KDM5A by siRNA significantly decreased caspase-3 activation in NCCs exposed to ethanol. The flow cytometry results indicated that the number of apoptotic cells was dramatically decreased in KDM5A deficient NCCs exposed to ethanol, as compared with the ethanol-treated group (Figure 5.10B). To determine whether knockdown of KDM5A prevented ethanol-induced apoptosis in vivo, zebrafish embryos were microinjected with control or KDM5A morpholino at one cell-stage, and treated with or without ethanol for 24 hours. TUNEL staining was used to determine the apoptotic cells in the whole zebrafish embryos. As shown in Figure $5.10 \mathrm{C}$, ethanol treatment resulted in excessive apoptotic cells in the forebrain, middle brain and hindbrain. Knockdown of KDM5A by microinjection with KDM5A morpholino attenuate ethanol-induced apoptosis in zebrafish embryos. 
a
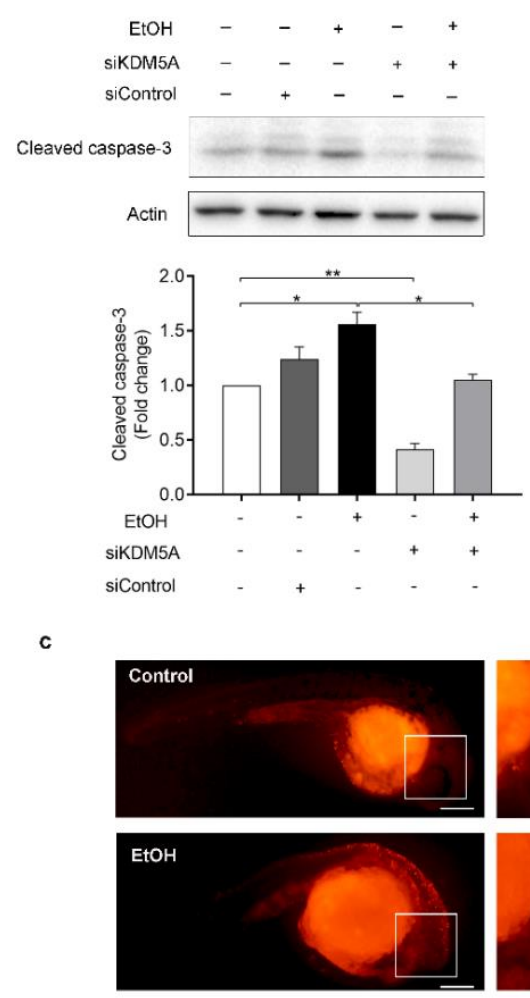

siKDM5A+EtOH

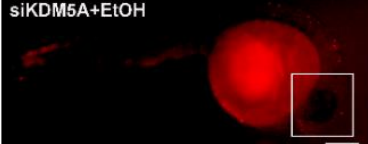

b
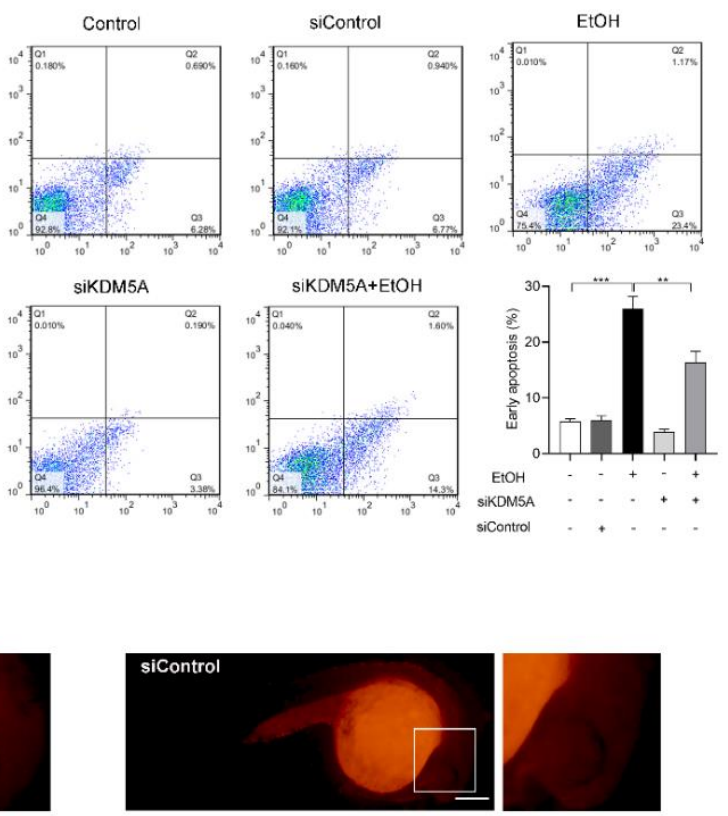

SiKDM5A
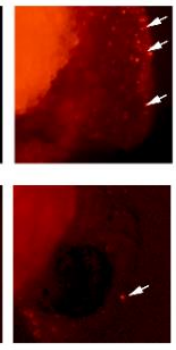

Figure 5. 10. The knockdown of KDM5A significantly decreased ethanol-induced apoptosis in NCCs and zebrafish embryos. NCCs transfected with control, or KDM5A siRNA were treated with or without $50 \mathrm{mM}$ ethanol for 24 hours. Apoptosis was determined by the analysis of caspase-3 cleavage by western blotting $(A)$ or flow cytometry with Annexin V-FITC apoptosis detection kit (B). Zebrafish embryos were microinjected with control or KDM5A morpholino at the one-cell stage and treated with or without for 24 hours. Apoptosis was analyzed by whole-mount TUNEL staining of the zebrafish embryos from control and treated groups. Scale bar: $200 \mu \mathrm{m}$. Data are expressed as fold change over control (A) and percentage of whole cells $(B)$ and represent the mean \pm SEM of three separated experiments. ${ }^{*} p<0.05,{ }^{* *} p<0.01$. 


\section{Discussion}

Apoptosis in NCCs is one of the major mechanisms underlying the pathogenesis of FASD. Recent studies have shown that SFN can epigenetically restore the expression of $\mathrm{Bcl}-2$ and attenuate ethanol-induced apoptosis by enhancing histone acetylation at the $\mathrm{Bcl}-2$ promoter ${ }^{18}$. The results from this study have shown that treatment with SFN significantly prevented ethanol-induced changes in the expression of E-cadherin and vimentin, and restored EMT in NCCs. I also found that ethanol exposure significantly reduced the levels of H3K4me3 at the promoter regions of Snail1. In addition, SFN treatment diminished the ethanolinduced reduction of $\mathrm{H} 3 \mathrm{~K} 4 \mathrm{me} 3$ at the promoter regions of the Snail1 gene, restored Snail1 gene expression and EMT. I have also found that the expression of KDM5A was increased by ethanol, which can be diminished by SFN treatment. Downregulation of KDM5A by siRNA significantly reduced ed ethanol-induced inhibition of $\mathrm{H} 3 \mathrm{~K} 4 \mathrm{me} 3$ expression as well as the $\mathrm{H} 3 \mathrm{~K} 4 \mathrm{me} 3$ enrichment at the promoter regions of the Snail1 gene. In addition, the Snail1 expression and EMT were restored in KDM5A deficient NCCs exposed to ethanol. Moreover, knockdown of KDM5A prevented ethanol-induced apoptosis in NCCs and zebrafish embryos.

It is well known that the EMT is a process that can inhibit apoptosis, promote the proliferation, migration and metastasis of tumor cells ${ }^{100,191,205,206}$. Study has shown that EMT conferred resistance to UV-induced apoptosis in murine mammary epithelial cell lines ${ }^{102}$. It has also been shown that $\alpha$-mangostin can inhibit EMT and induce apoptosis in an osteosarcoma cell line. EMT is also critical 
for the development of tissues and organs in the embryos ${ }^{93,95,97}$, and plays a crucial role in the regulation of the migration of $\mathrm{NCCs}^{10,91,207}$. However, the involvement of EMT in the ethanol-induced apoptosis in NCCs is currently unclear. I have demonstrated that ethanol treatment significantly inhibited EMT in NCCs, as indicated by an increased expression of E-cadherin and a decreased expression of vimentin, and induced apoptosis in NCCs. Treatment with SFN significantly diminished ed ethanol-induced changes in the expression of Ecadherin and vimentin, restored EMT, and reduced apoptosis in NCCs. These results suggest that inhibition of EMT contributes to ethanol-induced apoptosis in NCCs.

Snail1 is a member of the Snail superfamily of zinc-finger transcription factors that are involved in cell survival and differentiation ${ }^{104,105,208}$. Snail1 has a crucial role in the regulation of EMT through its repression of E-cadherin, an adhesion molecule mostly expressed in the surface of epithelial-like cells ${ }^{51}, 98,192,208,209$. Studies have shown that ethanol treatment decreased the expression of Snail1 mRNA and inhibited EMT in B16-BL6 melanoma cells ${ }^{210}$ and that Snail1 repressed TGF- $\beta$-induced apoptosis in hepatocytes by triggering EMT ${ }^{205}$. Ethanol exposure was also found to be able to down-regulate the Snail2 in NCCs of chick embryos 211. Consistent with these findings, I found that ethanol exposure resulted in a significant reduction in the expression of Snail1, accompanied by a dramatic increase in the expression of E-cadherin in NCCs. These results suggest that ethanol exposure can inhibit EMT in NCCs by down-regulating Snail1, leading to the up-regulation of E-cadherin. 
The expression of Snail1 can be regulated by a variety of mechanisms. Studies have shown that NF-kB, HIF-1a, SMAD, STAT3, and Gli1 can directly interact with the Snail1 promoter and regulate Snail1 at the transcriptional level ${ }^{192,}$ 206, 208. The expression of Snail1 can also be regulated epigenetically. In eukaryotic cells, epigenetic regulation of gene expression mainly comprises DNA modification and histone modification ${ }^{51,72,209}$. It has been reported that HDAC inhibitor valproic acid (VPA) elevated histone acetylation to transcriptionally activate Snail1 gene expression and promote EMT in colorectal cancer cells ${ }^{108}$. HDAC inhibitors, Trichostatin A (TSA) and Suberoylanilide hydroxamic acid (SAHA) also induced EMT in prostate cancer cells ${ }^{212}$. While acetylation of histone lysine residues can increase genome accessibility, thus promoting gene transcription, methylation at lysine residues can have either activating or repressing effects on gene transcription. It is well-known that trimethylation of histone 3 at lysine 27 (H3K27me3) is associated with transcription repression while trimethylation of histone 3 at lysine 4 (H3K4me3) is associated with transcription activation ${ }^{213}$. $\mathrm{H} 3 \mathrm{~K} 4 \mathrm{me} 3$ is highly enriched at active promoters near transcription start sites and is widely used as a histone mark to identify active gene promoters ${ }^{214}$. Interestingly, methylation of $\mathrm{H} 3 \mathrm{~K} 4$ is a kind of modifications that is associated with gene activation, implicated in neurodevelopment disorder ${ }^{194,198,199}$. In this study, I found that ethanol exposure resulted in a significant decrease in the levels of $\mathrm{H} 3 \mathrm{~K} 4 \mathrm{me} 3$ in NCCs and a significant reduction of $\mathrm{H} 3 \mathrm{~K} 4 \mathrm{me} 3$ enrichment at the promoter regions of Snail1. Co-treatment with SFN and ethanol significantly increased the H3K4me3 expression in NCCs and diminished the ethanol-induced reduction of 
H3K4me3 enrichment at the promoter regions of Snail1. These results indicate that the ethanol can induce apoptosis in NCCs by inhibiting EMT through epigenetically down-regulating the expression of Snail1 which can be prevented by SFN.

One of the key histone demethylases that remove the activating mark of H3K4me3 is KDM5A. KDM5A, also known as retinoblastoma binding protein 2 (RBP2), was associated with various cancer $^{215-217}$ and neurodevelopment disorder ${ }^{198,199}$. It has been shown that various HDAC inhibitors manifested a high inhibitory activity for $\mathrm{KDMs}^{200}$. Consistent with this finding, I found that SFN, an HDAC inhibitor, significantly decrease KDM5A in ethanol-exposed hNCCs. Furthermore, substantial studies have established that KDM5A can repress gene expression through maintaining the low levels of $\mathrm{H} 3 \mathrm{~K} 4 \mathrm{me} 3$ at gene promoter ${ }^{80,218-}$ 220. The present study indicated that ethanol-induced overexpression of KDM5A subsequently downregulated the H3K4me3 expression. In addition, knockdown of KDM5A by siRNA significantly increased H3K4me3 expression in ethanol-exposed NCCs. These results indicate that $\mathrm{H} 3 \mathrm{~K} 4 \mathrm{me} 3$ is the direct target of KDM5A in NCCs. In addition, the down-regulation of KDM5A by siRNA significantly reduced ethanolinduced reduction of $\mathrm{H} 3 \mathrm{~K} 4 \mathrm{me} 3$ enrichment at Snail1 promoter and subsequently restored Snail1 expression in NCCs. Furthermore, Snail1 is well recognized as a direct repressor of E-cadherin and can promote EMT process ${ }^{96,104,106,107 .}$. In the present study, I found that the down-regulation of KDM5A by siRNA diminished ethanol-induced increase in the expression of E-cadherin. These results indicated that the up-regulation of KDM5A represses the expression of Snail1 through the demethylation of H3K4me3 at Snail1 promoter, contributing to the inhibition of EMT 
in ethanol-exposed NCCs. The down-regulation of KDM5A by siRNA also significantly protected the NCCs from ethanol-induced apoptosis. Additionally, knockdown of KDM5A by microinjecting with morpholino KDM5A dramatically decreased ethanol-induced apoptotic cell death in the craniofacial position of zebrafish embryos. These results are consistent with the Klose's work that show that RBP2 (KDM5A) knockout mice displayed decreased apoptosis in hematopoietic stem cells ${ }^{80}$.

In summary, the present study indicated that ethanol exposure can inhibit EMT through the down-regulation of Snail1 by decreasing H3K4me3 enrichment at the promoter regions of Snail1 and increase apoptosis in NCCs. SFN treatment can diminish the ethanol-induced reduction in the $\mathrm{H} 3 \mathrm{~K} 4 \mathrm{me} 3$ enrichment at the promoter regions of Snail1, restore mRNA expression of Snail1 and EMT in NCCs exposed to ethanol. Ethanol exposure also significantly up-regulated KDM5A, which can be diminished by co-treatment with SFN. Furthermore, knockdown KDM5A by siRNA increased the expression of $\mathrm{H} 3 \mathrm{~K} 4 \mathrm{me} 3$ and $\mathrm{H} 3 \mathrm{~K} 4 \mathrm{me} 3$ enrichment at the Snail1 promoter. Down-regulation of KDM5A also restored Snail1 expression and EMT process and prevented apoptosis in ethanol-exposed NCCs. These results demonstrate that the disruption of EMT contributes to ethanol-induced apoptosis in NCCs and that SFN can prevent ethanol-induced apoptosis by restoring EMT through epigenetically regulating the expression of EMT-related genes, suggesting that elucidation of Snail1's role in EMT and ethanol-induced apoptosis in NCCs may provide critical insight into the pathogenesis of FASD. 


\section{CHAPTER VI}

MATERNAL DIETARY ADMINISTRATION OF BROCCOLI SPROUT EXTRACT DIMINISHED ETHANOL-INDUCED APOPTOSIS AND TERATOGENESIS IN MOUSE EMBRYOS BY EPIGENETICALLY MODULATING ANTI-APOPTOTIC

\section{GENES}

\section{A. Introduction}

The previous work utilizing animal models has shown that exposure to ethanol during the early stage of embryogenesis can result in craniofacial abnormalities that are characteristic of fetal alcohol syndrome disorder (FASD). Specifically, ethanol exposure could induce small head circumference, flat midface, short nose, indistinct philtrum, thin upper lip, short palpebral fissures and other defects ${ }^{221,222 .}$ Studies by Kotch and Sulik, as well as Dunty et al. in mice and Cartwright and Smith in chicks have revealed the sensitivity of the craniofacial region and developing brain to the deleterious effects of ethanol. These studies have shown that ethanol exposure during gastrulation and/or neurulation resulted in excessive apoptosis in neuroepithelium as well as other cell populations, including neural crest cells and ectodermal placodes ${ }^{14,130,131,156}$. The results of a whole embryo culture study by Van Maele-Fabry et al. also support the conclusion that neural crest cells and placodes are targets of ethanol's teratogenesis ${ }^{223}$. 
Furthermore, study by Debelak and Su et al. has shown that the severity of ethanol-induced neural crest apoptosis and craniofacial dysmorphology is influenced by genetics in an avian model of FASD 224,225 . Taken together, ethanolinduced apoptosis contributes to the dysmorphology of the craniofacial region and the developing brain in the FASD model. Therefore, preventing ethanol-induced apoptosis could be a promising therapeutic strategy for the prevention of FASD. Studies from our laboratory and others have shown that multiple signaling pathways are involved in ethanol-induced apoptosis, including Sonic Hedgehog $(\mathrm{SHH})$ signaling ${ }^{226}$, p53 pathways ${ }^{227}$, epithelial-mesenchymal transition (EMT) ${ }^{133}$ and $\mathrm{Bcl} 2$ family ${ }^{228}$. For instance, $\mathrm{SHH}$ is a potent cell survival factor and disruption in SHH signaling by ethanol may cause massive apoptosis in neural crest cells and neuroepithelial cells $226,229,230$. Studies have also shown that ethanol exposure caused oxidative stress, eventually triggering the apoptotic pathway $17,231,232$. The recent study from our laboratory has demonstrated that ethanol exposure significantly decreased the expression of Snail1, one of the major EMT transcriptional factors, and increased apoptosis in neural crest cells ${ }^{133}$. We have also shown that ethanol can induce apoptosis through epigenetically downregulating the anti-apoptotic gene, Bcl2, and subsequently lead to massive apoptosis in mouse embryos ${ }^{18}$. These results demonstrate that anti-apoptotic genes are crucial in regulating apoptosis and ethanol's teratogenesis. The previous study in our laboratory indicated that sulforaphane (SFN), found abundantly in broccoli and cruciferous vegetables, can reduce ethanol-induced apoptosis in both neural crest cells and mouse embryos ${ }^{17,18,133}$. More interestingly, 
studies have shown that SFN can epigenetically regulate apoptosis by acting as an inhibitor of histone deacetylase (HDAC) and DNA methyltransferase (DNMT), two key enzymes involved in histone deacetylation and DNA methylation ${ }^{18,}{ }^{133}$. Recently, broccoli sprout extract (BSE), has gained a lot of attention because it can specifically deliver a reproducible and standardized dose of glucoraphanin, a precursor of SFN. Glucoraphanin is available in commonly consumed cruciferous vegetables. BSE is considered to have low toxicity, and its administration in humans is well tolerated ${ }^{120-122}$. It was reported that dietary consumption of BSE can reduce the risk of several types of cancers, including prostate breast, lung and colorectal cancers $^{119,123,124}$. For instance, consumption of BSE inhibits HDAC activity in peripheral blood mononuclear cells, accompanied by the induction of acetylation of histone $\mathrm{H} 3$ and $\mathrm{H} 4$ in human subjects ${ }^{125}$. Moreover, evidence by Hashimoto et al. has shown that dietary intake of SFN-rich BSE was capable of preventing PCP-induced cognitive deficits and oxidative stress in mice ${ }^{126}$. Furthermore, SFN-rich BSE significantly improves social interaction, abnormal behavior and verbal communication in young men with autism spectrum disorders $^{120}$.

For the present investigation, I tested the hypothesis that maternal dietary administration of BSE can prevent ethanol-induced apoptosis and teratogenesis through epigenetically regulating the anti-apoptotic genes. Through using the in vivo oral take FASD model system, I found that the anti-apoptotic genes were hypermethylated by maternal consumption of ethanol, which can be diminished by maternal dietary administration of BSE. Subsequently, maternal dietary BSE 
diminished ethanol-induced down-regulation of the anti-apoptotic genes. Moreover, BSE consumption prevented ethanol-induced apoptosis in mouse embryos. Furthermore, I found that maternal consumption of BSE conferred in vivo protection against ethanol-induced teratogenesis, including the anterior tube defects and ocular abnormalities in mouse embryos. These results demonstrate that epigenetic modulation of the anti-apoptotic genes by maternal dietary SFNrich BSE represents a novel therapeutic strategy for the prevention of ethanolinduced teratogenesis.

\section{B. Experimental Procedures}

\section{Animals and treatments}

Animals were maintenance, treated with ethanol and BSE, as described in Chapter II Section B.1 and in the Experimental scheme 6.1. 


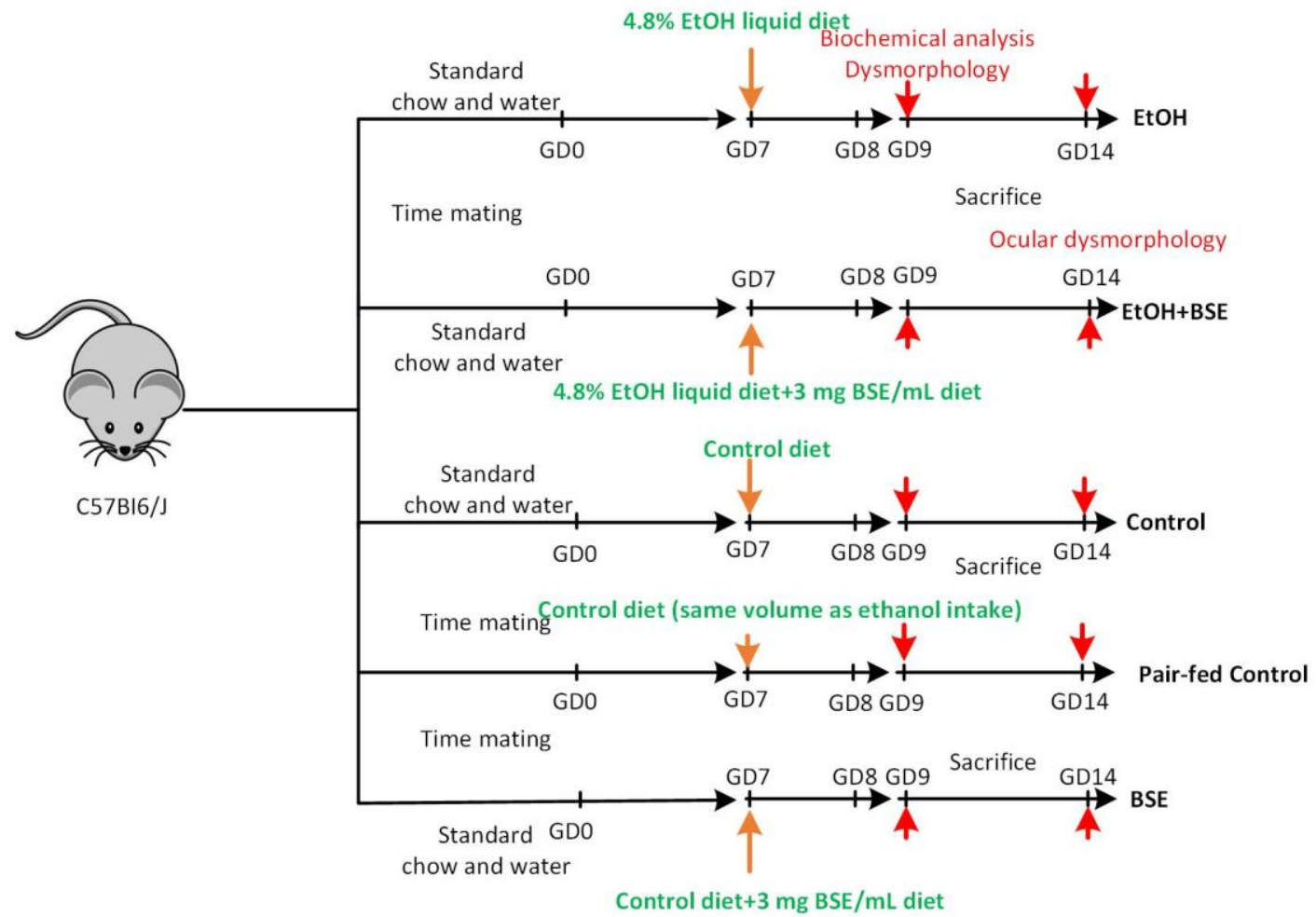

Experimental scheme 6. 1. FASD mouse model and experimental procedure. Mice received ethanol-containing or control liquid diet for an initial 14 days then moved to the lab chow for mating. $4.8 \%$ ethanol liquid diet with or without $3 \mathrm{mg} \mathrm{BSE} / \mathrm{mL}$ diet was re-introduced to the experimental groups of pregnant mice on GD 7.0 and GD 8.0. Pregnant mice will be killed on GD 9.0 or GD 14.0 for further analysis (see Experimental procedure).

\section{Methylated specific PCR (MSP)}

Pregnant mice were sacrificed on GD 9 and the whole brain was dissected from the mouse embryos from control and experimental groups. The genome DNA was isolated from the whole brain of embryos from control and experimental groups, and DNA methylation at the promoters of the anti-apoptotic genes was determined as described in Chapter II, C. 6. 


\section{Dysmorphology assessment}

Pregnant mice were sacrificed on GD 9, and the embryos were dissected and kept in ice-cold PBS. The embryos were then photographed under a microscope (SZX16, Olympus, USA). Ocular dysmorphology was assessed at GD 14. The pregnant mice were sacrificed on GD 14, and the embryos were dissected from the uterus. The eyes were photographed under a microscope (SZX16, Olympus, USA).

\section{TUNEL Staining}

Analysis of apoptosis in mouse embryos was performed by using a TMR Red in Situ Detection Kit (Millipore, Temecula, CA), following the manufacturer's protocol. Briefly, the mouse embryos were fixed with $4 \%$ paraformaldehyde overnight, then dehydrated in $100 \%$ methanol at $-20 \stackrel{\circ}{ } \mathrm{C}$ for at least 2 hours, following by rehydrating the embryos through a series of methanol dilutions. Then the embryos were permeabilized with proteinase $\mathrm{K}$ at room temperature for $8 \mathrm{~min}$ following with $0.1 \%$ Triton- 100 in $0.1 \%$ sodium citrate treatment at room temperature for an additional $15 \mathrm{~min}$. After that, the embryos were pre-fixed with 4\% paraformaldehyde for $20 \mathrm{~min}$ and then stained with the TUNEL reaction mixture at $37^{\circ} \mathrm{C}$ for 1 hour. Following the staining, the mouse embryos were visualized in the green channel of a fluorescent microscope (SZX16, Olympus, USA).

\section{Quantitative real-time PCR}


mRNA expression of AKT1, BIRC5, BCl2, and NAIP were determined by quantitative real-time PCR, as described in Chapter II, Section C.2.

\section{Western Blot}

The protein level of AKT1, BIRC5, BCl2, NAIP, and Cleaved caspase-3, were determined by western blot, as described in Chapter II, Section C.3.

\section{Statistical analysis}

Statistical analysis was performed, as described in Chapter II, C. 8.

\section{C. $\underline{\text { Results }}$}

1. Maternal dietary BSE administration significantly reduced ethanolinduced hypermethylation at the promoters of the selected antiapoptotic genes in mouse embryos.

To determine whether maternal dietary BSE can reduce ethanol-induced hypermethylation at the promoter regions of the anti-apoptotic genes in mouse embryos exposed to ethanol, the whole brain was dissected from GD 9.0 mouse embryos, and the methylation levels at the promoter regions of the anti-apoptotic genes were analyzed. As shown in Figure 6.1, maternal dietary BSE significantly diminished ethanol-induced hypermethylation at the promoters of AKT1, Bcl2 and NAIP in mouse embryos. 
A
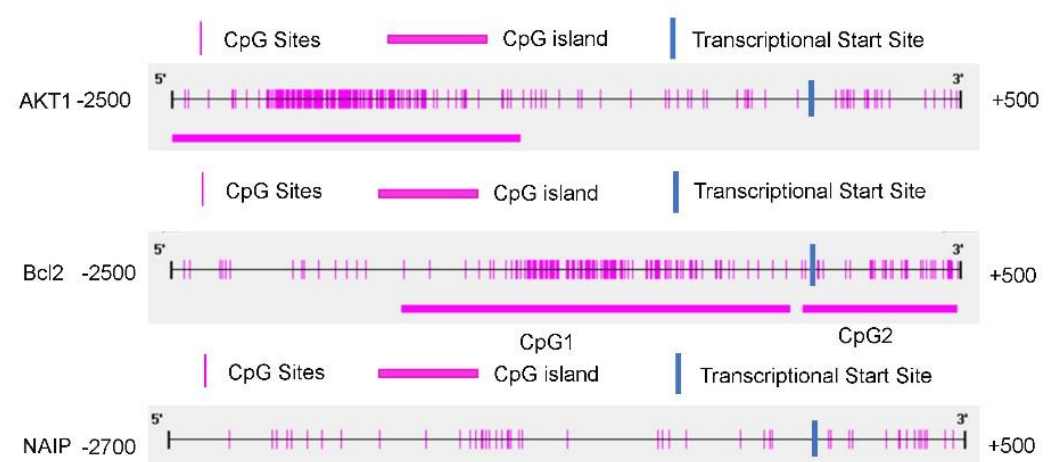

B
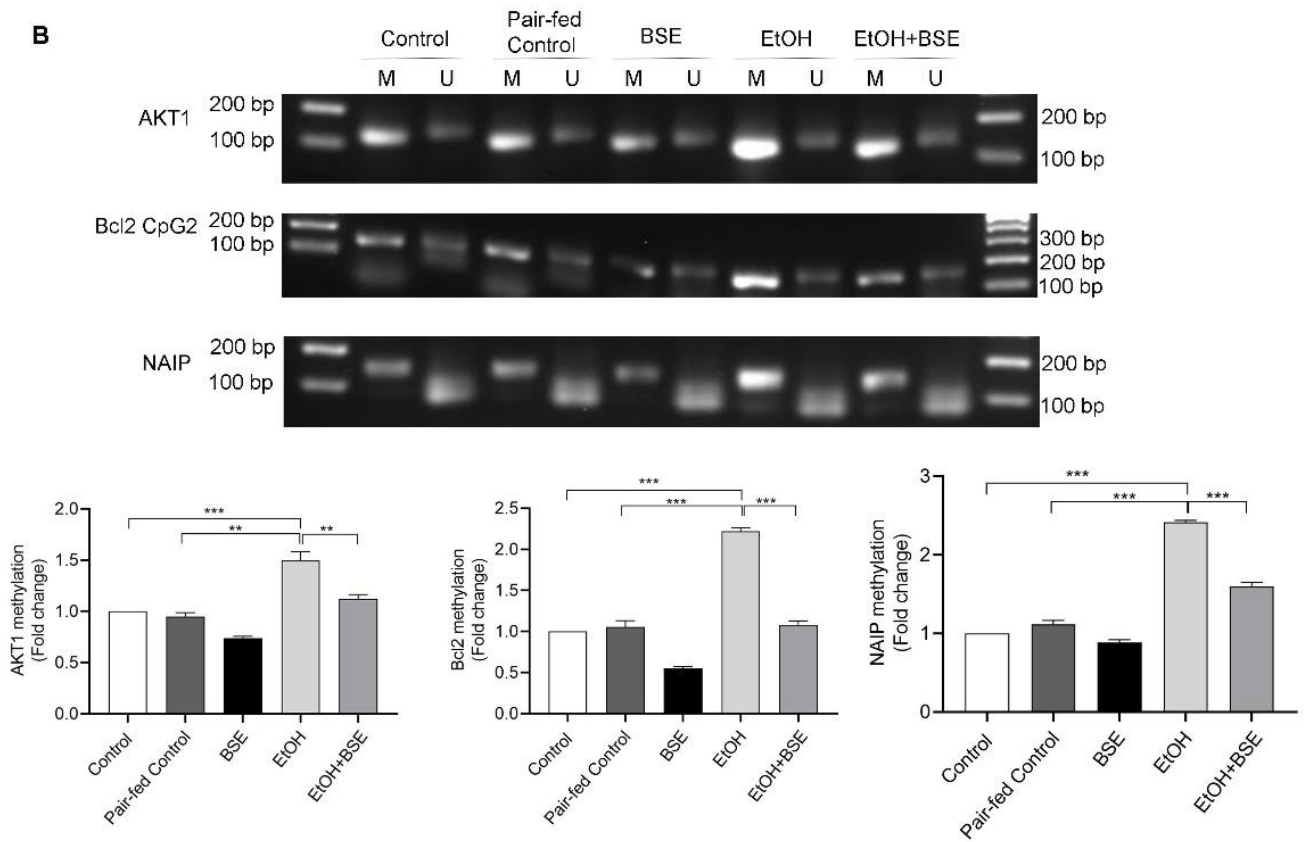

Figure 6. 1. Maternal dietary BSE significantly diminished ethanol-induced hypermethylation at the promoters of AKT1, Bcl2 and NAIP in mouse embryos. Control and treated mice were sacrificed at GD 9.0. The whole brain was dissected from the embryos and the genome was isolated. (A) Schematic illustration of $\mathrm{CpG}$ sites and CpG island at the promoters of the anti-apoptotic genes. (B) DNA methylation status at the $\mathrm{CpG}$ island of the selected anti-apoptotic genes was determined by using MSP. Data are expressed fold change over control and represent the mean \pm SEM of three separated experiments. ${ }^{*} p<0.05,{ }^{* *} p<0.01$, ${ }^{\star * *} p<0.001$. 


\section{Maternal dietary BSE reduced the ethanol-induced down-regulation of the selected anti-apoptotic genes in mouse embryos}

DNA methylation is the most extensively studied epigenetic modification, which has an important role in the regulation of gene expression. DNA hypermethylation on CpG island compacts the chromatin, silencing the gene expression. Conversely, when DNA methylation is lowered, the chromatin is allowed to open and subsequently leads to gene activation ${ }^{233,234}$. To determine whether the diminished hypermethylation at the promoter region of the antiapoptotic genes induced by maternal dietary BSE in ethanol-exposed mouse embryos can reduce ethanol-induced down-regulation of the selected antiapoptotic genes, the expression of the anti-apoptotic genes was determined by qRT-PCR and western blot. As expected, ethanol treatment alone robustly decreased the mRNA expression of AKT1, Bcl2 and NAIP. BSE administration significantly diminished the ethanol-induced down-regulation of these antiapoptotic genes (Figure 6.2A). Moreover, maternal dietary BSE also significantly diminished ethanol-induced decrease in the protein expression of AKT1, Bcl2 and NAIP in mouse embryos (Figure 6.2B). These results demonstrate that maternal dietary BSE can prevent ethanol-induced down-regulation of the anti-apoptotic genes. 
a
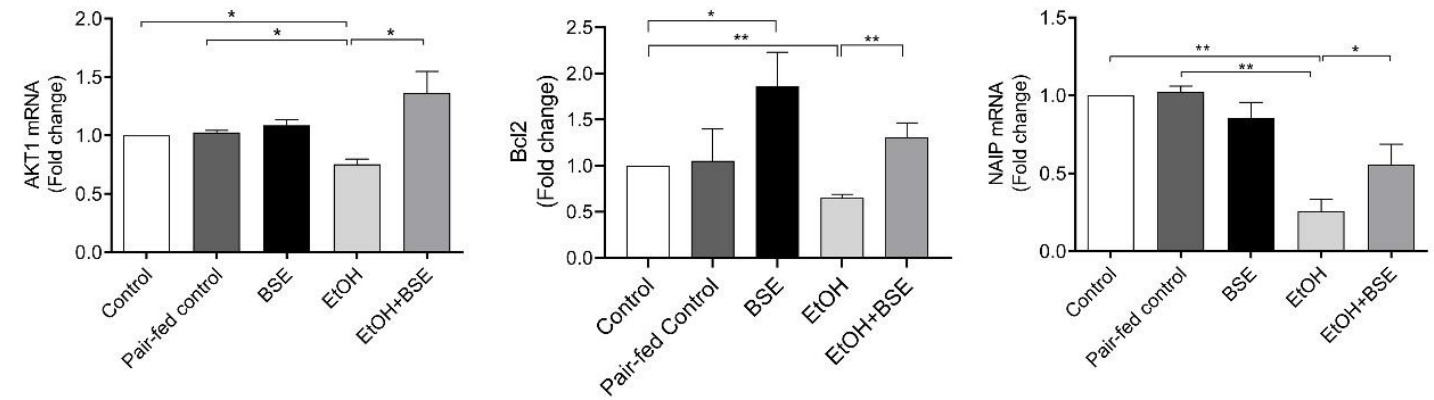

b

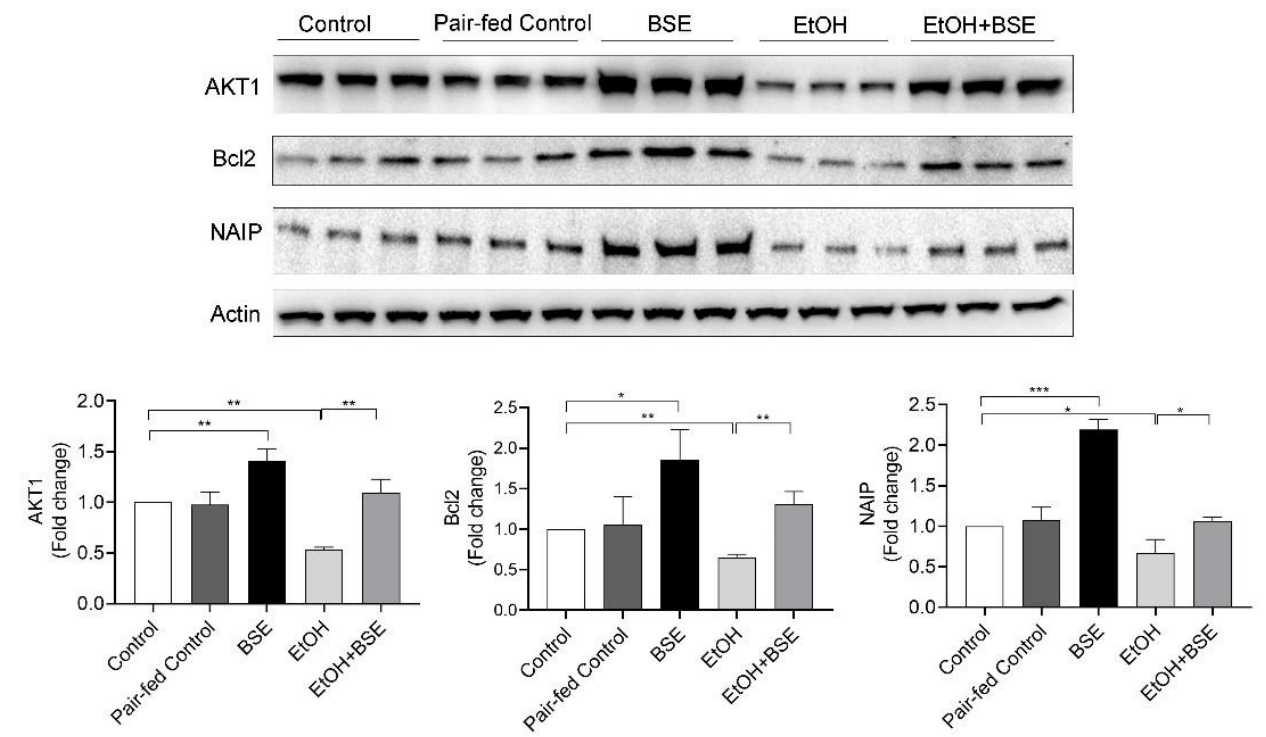

Figure 6. 2. Maternal dietary BSE prevented ethanol-induced down-regulation of anti-apoptotic genes in mouse embryos. The control and treated mice were sacrificed at GD 9.0, and then the whole brain was dissected from the mouse embryos. The mRNA (A) and protein (B) expression of the anti-apoptotic genes were analyzed by quantitative RT-PCR and Western blot, respectively. Data are expressed as fold change over control and represent the mean \pm SEM of three separated experiments. ${ }^{*} p<0.05,{ }^{* *} p<0.01,{ }^{* * *} p<0.001$. 


\section{Maternal dietary BSE protected against ethanol-induced apoptosis in mouse embryos}

To determine whether maternal dietary BSE can prevent ethanol-induced apoptosis, apoptosis was examined by analysis of caspase- 3 activation and wholemount TENEL staining in control and treated mouse embryos. As shown in Figure 6.3A, ethanol exposure resulted in a significant increase in caspase-3 activation, indicating that ethanol exposure increased apoptosis in mouse embryos. BSE administration significantly reduced caspase-3 activation in mouse embryos exposed to ethanol. Moreover, the apoptotic cells were evaluated by whole-mount TUNEL staining of control and treated GD 9.0 mouse embryos. As compared to control group, ethanol-exposed mouse embryos displayed excessive apoptosis in the hindbrain with staining most intensely in the hindbrain alar plate, additional locations of excessive apoptosis were observed in the forebrain, middle brain (white arrow), trigeminal ganglion (V) as well as first arch (I). BSE administration significantly attenuated ethanol-induced excessive apoptosis in mouse embryos (Figure 6.3B). These results demonstrate that maternal dietary BSE can prevent ethanol-induced apoptosis in mouse embryos. 

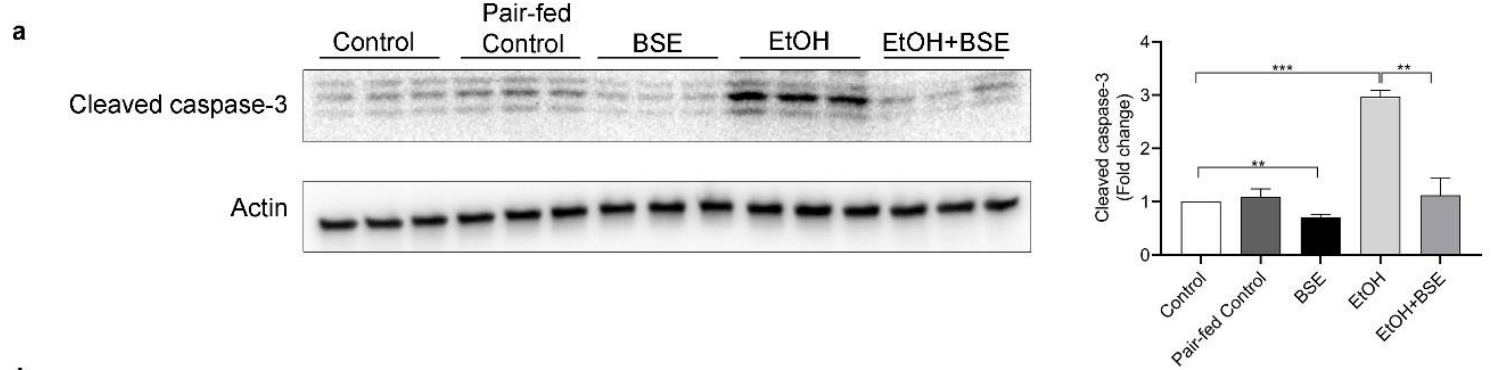

b

Control

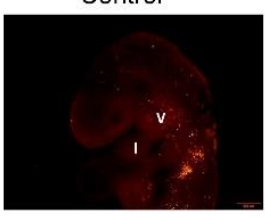

Pair-fed Control

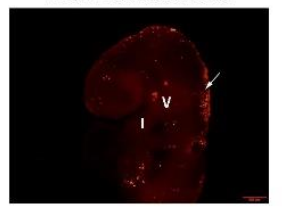

BSE

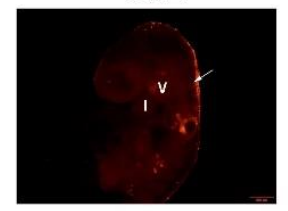

$\mathrm{EtOH}$

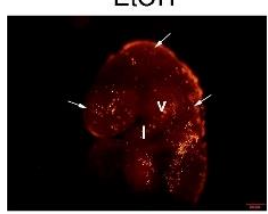

EtOH+BSE

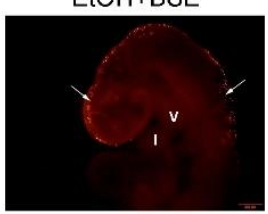

Figure 6. 3. Maternal dietary BSE protects against ethanol-induced apoptosis in mouse embryos. The control and treated mice were sacrificed at GD 9.0, and the mouse embryos were dissected. (A) Apoptosis was determined by the analysis of caspase-3 cleavage using Western blot. (B) The whole-mount TUNEL staining of mouse embryos from control and treated groups was visualized under a fluorescence microscope. Data are expressed as fold change over control and represent the mean \pm SEM of three separated experiments. ${ }^{* *} p<0.01{ }^{* * *} p<0.001$. I=first arch; V=trigeminal ganglion. 


\section{Maternal dietary BSE confers in vivo protection against ethanol- induced teratogenesis in mouse embryos.}

To determine whether dietary consumption of BSE can prevent ethanolinduced teratogenesis, the morphology of the control and treated mouse embryos was assessed on GD 9.0. As compared with control groups, ethanol-exposed mouse embryos displayed a marked decrease in the size of the forebrain, midbrain and hindbrain, BSE administration reduced ethanol-induced brain growth retardation on GD 9.0. Moreover, the size of the first branchial arches was reduced in the ethanol-treated group, as compared with the control group. BSE treatment prevented the ethanol-induced decrease in the size of the first arches. (Figure 6.4A). Additionally, ethanol exposure alone significantly decreased the number of live embryos, somite number and crown-rump length in GD 9.0 mouse embryos. Moreover, ethanol treatment also significantly increased the incidence of neural tube defects in mouse embryos. BSE administration significantly ameliorated ethanol-induced growth retardation and abnormalities in GD 9.0 mouse embryos. 

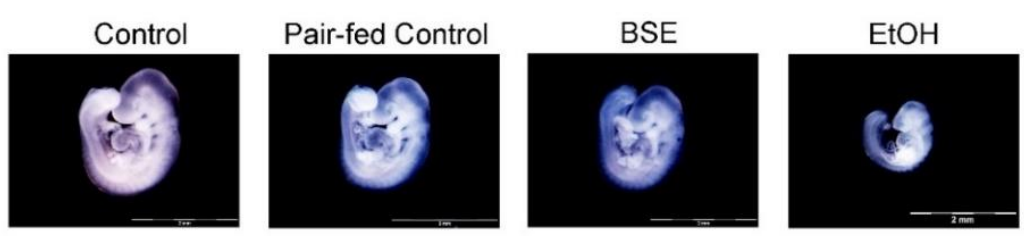

$\mathrm{EtOH}+\mathrm{BSE}$
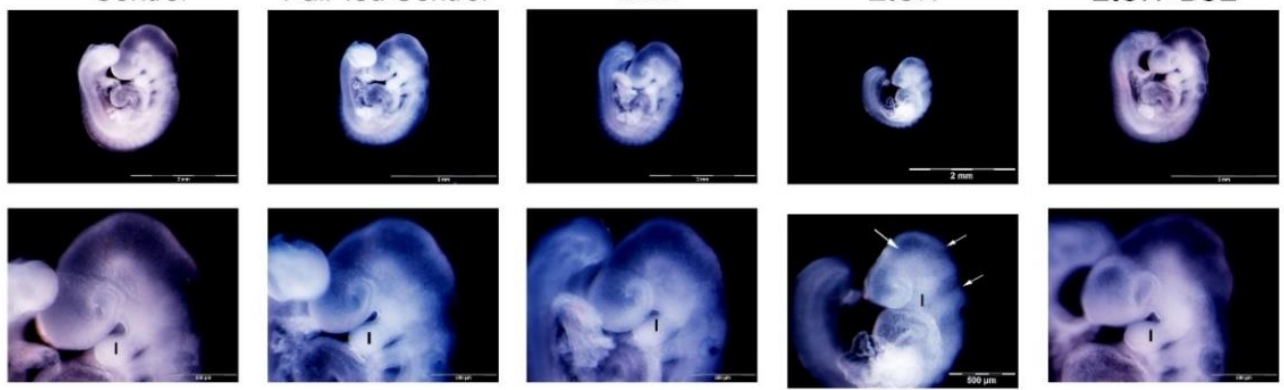

b
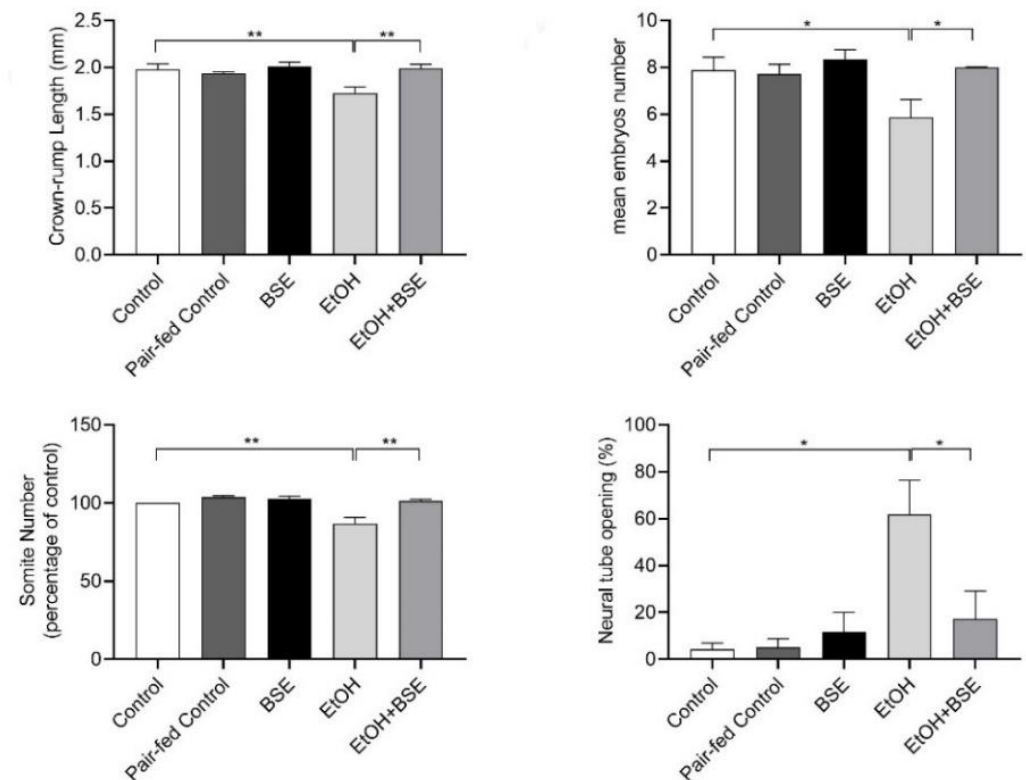

Figure 6. 4. BSE administration confers in vivo protection against ethanol-induced teratogenesis. Control and treated groups were sacrificed at GD 9.0 and the mouse embryos were dissected. (A) Illustrated are the mouse embryos of control and treated groups showing the ethanol-induced growth retardation and neural tube defects and the protective effects of BSE on the ethanol-induced growth retardation and neural tube defects. (B) Statistic analysis of the number of live embryos somite number, crown-rump length and neural tube defects in GD 9.0 mouse embryos. Data are expressed as mean \pm SEM. ${ }^{*} p<0.05,{ }^{* *} p<0.01$. 


\section{Maternal dietary administration of BSE prevented ethanol-induced ocular abnormalities in mouse embryos.}

To determine whether BSE adminstration can prevent ethanol-induced ocular abnormalities, the control and treated mice were sacrifiered at GD 14, and the ocular abnormalities were examined. As shown in Figure 6.5A, ethanol exposure significantly increased the incidence of abnormalities in the right eyes. Additionally, using the rating scale described in Figure 6.5B, I found that ethanol administration increased the severity of ocular defects as compared with the control group, with $24 \%$ of the right eyes in the ethanol group being mildly affected (a rating of 2), whereas $6 \%$ of the eyes were both slightly microphthalmic and had abnormally shaped pupils (a rating of 3 ), $3 \%$ of the eyes were moderately microphthalmic (a rating of 4 ), and $3 \%$ were severely microphthamic (a rating of 5). Concurrent exposure of the pregnant mice to BSE and ethanol resulted in a lower incidence and severity of ocular abnormalities compared with ethanol alone group. The incidence of eye defects in the group treated with both ethanol andBSE was reduced by $21 \%$ in the right eyes as compared with that in ethanol alone group. There were no fetuses with the moderate-severe (rating of 4) or the most severe (rating of 5) ocular defects in the group treated with both ethanol and BSE (Figure $6.5 \mathrm{C})$. 

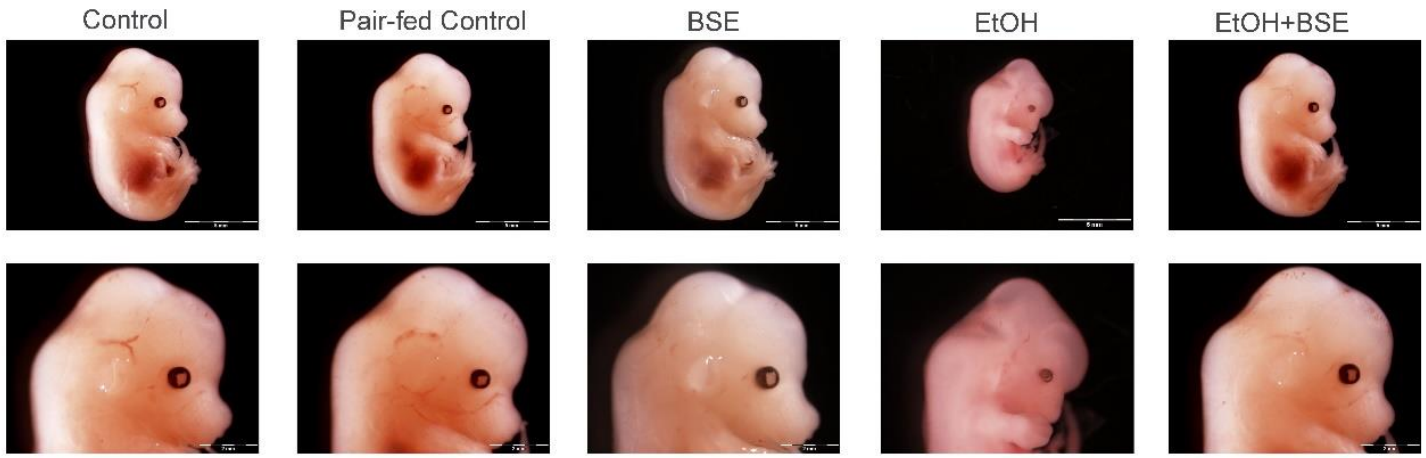

b

Range of Ocular defect
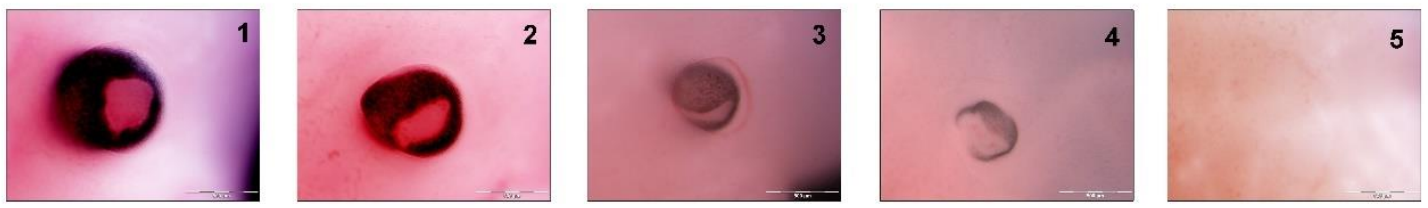

c

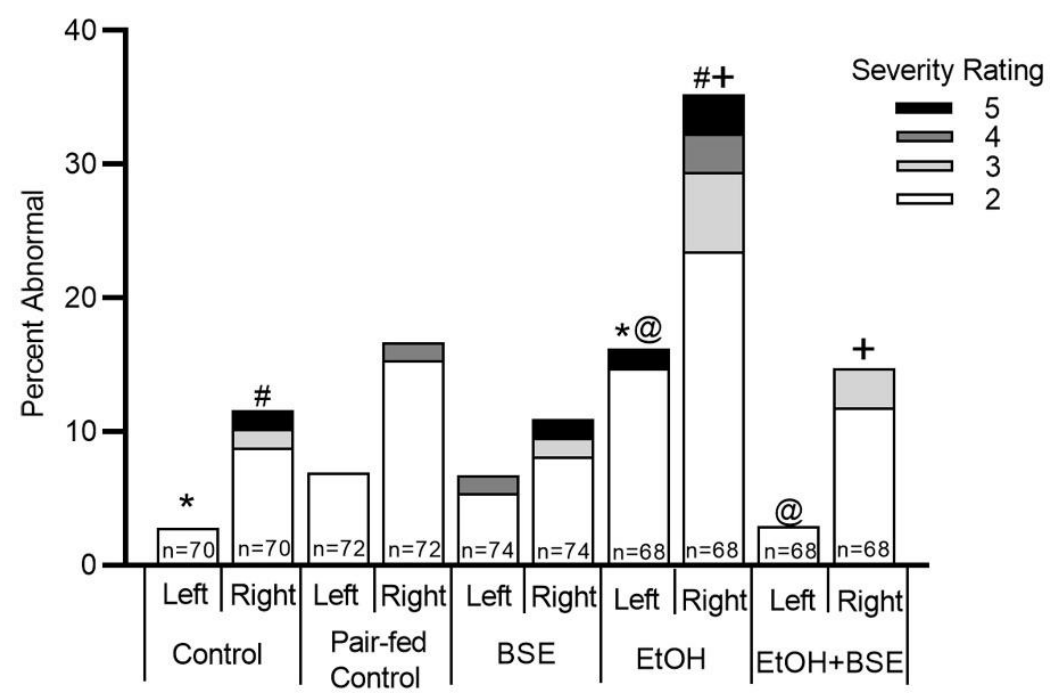

Figure 6. 5. Maternal dietary administration of BSE prevented ethanol-induced ocular abnormalities in mouse embryos. The control and experimental mice were sacrificed at GD 14.0 and the mouse embryos were dissected for assessment. (A) Ocular dysmorphology was visualized under a microscope. (B) Range of ocular defects. The eyes were rated according to the following categories: normal eye (rating of 1) (1); slightly abnormal pupil shape (rating of 2) (2); mild microphthalmia and slightly abnormal pupil shape (rating of 3) (3); moderate microphthalmia (rating 
of 4) (4); and severe microphthalmia (rating of 5) (5). (C) Incidence and severity of ocular defects. Ethanol exposure resulted in significant increases in both the incidence and severity of ocular abnormalities in both the left $\left(^{*}\right)$ and the right eyes (\#). Concurrent exposure to ethanol and BSE resulted in a lower incidence of ocular defects in both the left (@) and right $(+)$ eyes compared with ethanol alone groups. 


\section{Discussion}

Apoptosis plays an important role in ethanol-induced teratogenesis. Recent studies have shown that SFN can attenuate ethanol-induced apoptosis through epigenetically up-regulating the anti-apoptotic genes, Bcl2 andSnail1 ${ }^{18,133}$. In this study, I have found that maternal consumption of BSE significantly reduced ethanol-induced down-regulations of the anti-apoptotic genes, AKT1, Bcl-2 and NAIP, in mouse embryos through diminishing ethanol-induced hypermethylation at the promoter regions of these anti-apoptotic genes. I have also found that BSE administration diminished ethanol-induced apoptosis in mouse embryos, as indicated by the reduction of cleaved caspase-3 expression. TUNEL staining also revealed that the apoptosis in the brain and first branchial arches was significantly reduced in ethanol and BSE co-treatment group as compared with ethanol alone group. Maternal dietary BSE also significantly ameliorated ethanol-induced growth retardation, neural tube defects and ocular abnormalities in mouse embryos.

SFN can modulate gene expression by inhibition of HDAC and DNMT, two enzymes associated with histone deacetylation and DNA methylation. The previous study in our lab has demonstrated that SFN increased acetyl-histone $\mathrm{H} 3$ binding to the Bcl-2 promoter, reduced ethanol-induced apoptosis in both neural crest cells and mouse embryos ${ }^{18}$. Moreover, study has also indicated that SFN inhibited HDAC activity in human colon ${ }^{235}$ and prostate cancer cell liness and resulted in an increase in global and local histone acetylation status on the promoter region of apoptosis-related genes ${ }^{244}$. In addition to modulating HDACs and histone acetylation, SFN can also regulate DNA methylation by acting as an 
inhibitor of DNMTs. It was reported that SFN suppressed DNA methylation in the hTERT promoter, leading to transcriptional repression in breast cancer cells ${ }^{236}$. Another study by Anna et al. has shown that SFN significantly decreased enzymatic activity of DNMTs and decreased methylation on cyclin D2 promoter regions in prostate cancer cells ${ }^{237}$. SFN is a compound produced by the conversion of glucoraphanin in the presence of the myrosinase enzyme ${ }^{238}$. Increased amounts of glucoraphanin can enhance SFN production in broccoli or broccoli sprouts. BSE contains up to 50 times higher concentrations of glucoraphanin than mature broccoli ${ }^{239}$. Consumption of BSE has been found to significantly increase isothiocyanate levels in human plasma. Additionally, it has also been demonstrated that SFN can cross the placenta during maternal dietary supplementation of BSE between GD 9.0 to GD 19.0 in mice. In human subjects, a single dose of $68 \mathrm{~g}$ BSE significantly inhibited HDAC activity in peripheral blood mononuclear cells 3 hours following treatment, with the increased induction of acetylation of histones $\mathrm{H} 3$ and $\mathrm{H} 4{ }^{125}$. However, the dietary BSE on DNA methylation is not well known. In the present study, I found that maternal dietary BSE reduced the ethanol-induced hypermethylation at the promoter regions of the anti-apoptotic genes, including AKT1, Bcl-2, and NAIP, in mouse embryos exposed to ethanol. In addition, SFN-rich BSE-mediated reduction of hypermethylation at the promoter regions of these anti-apoptotic genes significantly diminished ethanol-induced down-regulation of these genes in mouse embryos. Maternal dietary BSE also prevented ethanol-induced apoptosis in mouse embryos exposed to ethanol, as evidenced by the reduction of caspase-3 
activation and TUNEL staining in the group co-treated with BSE and ethanol. These results are consistent with the study showing that SFN-enriched BSE inhibited neuronal apoptosis through inhibition of caspase-3 activation, leading to the neuroprotection against neurodegenerative disorders ${ }^{240}$.

Children with FASD display characteristic craniofacial anomalities, including short palpebral fissures, hypoplastic midface, deficient philtrum and long upper lip 241,242 . Research by Dunty and Sulik has shown ethanol-induced apoptosis of specific progenitor cells leads to craniofacial deficits associated with FASD ${ }^{130}$. Consistent with these findings, I found that ethanol-exposed GD 9.0 mouse embryos displayed the short first branchial arch and reduced brain size, which is also consistent with Scott and Sulik's study that has shown that the size of the entire forebrain in ethanol exposed mouse embryos was significantly reduced ${ }^{243}$. Maternal dietary BSE significantly diminished the ethanol's effects on the first branchial arch and brain size in mouse embryos. In addition, BSE administration significantly prevented the ethanol-induced reduction of the number of live embryos, somite number, and crown-rump length and ethanol-induced neural tube defects. These results demonstrate that maternal dietary administration of BSE can prevent ethanol-induced growth retardation and abnormalities in GD 9.0 mouse embryos.

In the present study, ocular dysmorphology on GD 14.0 in mouse embryos was also selected as the key morphological endpoint for analysis. The ocular defects induced by ethanol were known to be involved in diminished global size and pupil abnormalities ${ }^{243}$. In this study, ethanol exposure caused $36 \%$ increase 
in the ocular defects at the right eye and $16 \%$ increase in the left eye. This result is consistent with the previous studies usingan FASD model that has shown that the right eyes were affected at a markedly higher incidence than the left eyes ${ }^{231}$, 243, 244. In ethanol and BSE co-treatment group, the defects of the right eye were reduced by $21 \%$ as compared with ethanol alone group. These results demonstrate that BSE can significantly diminish ethanol-induced ocular defects in mouse embryos.

In summary, this study indicated that the promoter region of the anti-apoptotic genes, includingAKT1, Bcl2 and NAIP, were hypermethylated in ethanol-exposed mouse embryos, which can be diminished by BSE administration. Maternal dietary BSE also diminished ethanol-induced down-regulation of the selected antiapoptotic genes and prevented ethanol-induced apoptosis in mouse embryos. In addition, maternal dietary BSE significantly attenuated ethanol-induced growth retardation and neural tube defects in GD 9.0 mouse embryos and ethanol-induced ocular abnormalities in GD 14.0 mouse embryos. These findings provide insights into the epigenetic mechanisms involved in ethanol-induced apoptosis, growth retardation and teratogenesis in mouse embryos, suggesting that the epigenetic modulation of anti-apoptotic genes by using SFN-rich BSE might be a promising therapeutic strategy for the prevention of FASD. 


\section{CHAPTER VII}

\section{DISCUSSION AND CONCLUSIONS}

\section{A. Restatement of goals}

The overall goal of the work described in this dissertation was to elucidate the epigenetic mechanisms by which SFN epigenetically regulates the anti-apoptotic gene expression and prevents ethanol-induced apoptosis and teratogenesis, as well as to develop new therapeutic approaches for the prevention of FASD. The experiments described in Chapter III were performed to determine whether SFN prevents the hNCCs from ethanol-induced apoptosis through altering the DNA methylation level at the promoter regions of the anti-apoptotic genes. The study described in Chapter IV tested the hypothesis that SFN can prevent ethanolinduced apoptosis by modulating histone acetylation at the promoter region of the anti-apoptotic genes. The work described inn Chapter Vtested the hypothesis that SFN can protect against ethanol-induced apoptosis by restoring EMT through epigenetically modulating the expression of Snail1 in NCCs and that downregulation of KDM5A can prevent ethanol-induced apoptosis in NCCs and in zebrafish embryos. The study described in Chapter VI tested the hypothesis that maternal dietary administration of BSE can prevent ethanol-induced apoptosis and teratogenesis through epigenetically regulating the anti-apoptotic genes. Taken 
together, these studies provide novel information on the epigenetic mechanisms of ethanol-induced apoptosis and teratogenesis. These findings also suggest that epigenetic modulation of anti-apoptotic genes by SFN or BSE might be a promising strategy for the prevention of FASD.

\section{B. Major findings of this dissertation}

1. SFN protects against ethanol-induced apoptosis in hNCCs through diminishing ethanol-induced hypermethylation at the promoters of the anti-apoptotic genes.

Our studies described in Chapter III indicated that ethanol treatment can inhibit the expression of the anti-apoptotic genes, including AKT1, Bcl2, BIRC5, BIRC6, IGF1R, MCL1, NAIP and XIAP and that ethanol-induced repression of these anti-apoptotic genes can be prevented by SFN. We have also shown that ethanol exposure resulted in a significant increase in the DNMT activity and the expression of DNMT3a in hNCCs. SFN can significantly diminish ethanol-induced increases in DNMT activity and the expression of DNMT3a. We have also found that ethanol-induced up-regulation of DNMT3a and an increase in DNMT activity resulted in hypermethylation at the promoters of the selected anti-apoptotic genes in hNCCs and that SFN can diminish ethanol-induced hypermethylation at the promoters of anti-apoptotic genes by preventing ethanol-induced up-regulation of DNMT3a and increase in DNMT activity. In addition, the knockdown of DNMT3a or treatment with SFN significantly diminished ethanol-induced decreases in the mRNA and protein expression of NAIP and XIAP and prevented ethanol-induced apoptosis in hNCCs. The knockdown of DNMT3a also enhanced the effects of 
SFN on the mRNA and protein expression of NAIP and XIAP and the protective effects of SFN on ethanol-induced apoptosis in hNCCs

2. SFN prevents hNCCs from ethanol-induced apoptosis by diminishing ethanol-induced reduction of histone acetylation at the promoters of the anti-apoptotic genes.

Our studies described in Chapter IV have indicated that ethanol exposure can increase HDAC activity and the expression of HDAC2 in hNCCs. SFN treatment significantly diminished ethanol-induced increase in HDAC activity and the up-regulation of HDAC2. We have also found that ethanol-induced increase in HDAC activity and up-regulation of HDAC2 resulted in the reduction of $\mathrm{H} 3$ acetylation at the promoters of AKT1, BIRC6 and XIAP in hNCCs and that SFN diminished ethanol-induced reduction of $\mathrm{H} 3$ acetylation at the promoters of antiapoptotic genes by inhibiting HDAC activity and reducing ethanol-induced upregulation of HDAC2. In addition, SFN treatment or knockdown of HDAC2 significantly diminished ethanol-induced decreases in the mRNA and protein expression of AKT1, BIRC6 and XIAP and prevented ethanol-induced apoptosis in hNCCs. The knockdown of HDAC2 also enhanced the effects of SFN on the mRNA and protein expression of AKT1, BIRC6 and XIAP and the protection against ethanol-induced apoptosis in hNCCs.

3. SFN protects against ethanol-induced apoptosis in NCCs and zebrafish embryos through modulating histone methylation and the expression of Snail 1 and restoring EMT. 
Our studies presented in Chapter $\mathrm{V}$ indicated that ethanol exposure can inhibit EMT through the down-regulation of Snail1 by decreasing H3K4me3 enrichment at the promoter regions of Snail1 and increase apoptosis in NCCs. SFN treatment can diminish the ethanol-induced reduction in the H3K4me3 enrichment at the promoter regions of Snail1, restoring mRNA expression of Snail1 and EMT in NCCs exposed to ethanol. Ethanol exposure also significantly upregulated KDM5A, which can be diminished by co-treatment with SFN. Furthermore, knockdown KDM5A by siRNA increased the expression of $\mathrm{H} 3 \mathrm{~K} 4 \mathrm{me} 3$ and H3K4me3 enrichment at the Snail1 promoter. Down-regulation of KDM5A also restored Snail1 expression and EMT process and prevented apoptosis in ethanolexposed NCCs. These results demonstrate that the disruption of EMT contributes to ethanol-induced apoptosis in NCCs and that SFN can prevent ethanol-induced apoptosis by restoring EMT through epigenetically regulating the expression of EMT-related genes, suggesting that elucidation of Snail1's role in EMT and ethanol-induced apoptosis in NCCs may provide critical insight into the pathogenesis of FASD.

\section{Maternal dietary administration of SFN-rich BSE diminished ethanol- induced apoptosis and teratogenesis in mouse embryos by epigenetically modulating anti-apoptotic genes.}

The study described in Chapter VI indicated that the promoter region of the anti-apoptotic genes, including AKT1, Bcl2 and NAIP, were hypermethylated in ethanol-exposed mouse embryos, which can be diminished by BSE administration. Maternal dietary BSE also diminished ethanol-induced down-regulation of the 
selected anti-apoptotic genes and prevented ethanol-induced apoptosis in mouse embryos. In addition, maternal dietary BSE significantly attenuated ethanolinduced growth retardation and neural tube defects in GD 9.0 mouse embryos and ethanol-induced ocular abnormalities in GD 14.0 mouse embryos. These findings

provide insight into the epigenetic mechanisms involved in ethanol-induced apoptosis, growth retardation and teratogenesis in mouse embryos, suggesting the epigenetic modulation of anti-apoptotic genes by using SFN-rich BSE might be a promising therapeutic strategy for the prevention of FASD.

\section{Significance}

FASD is among the most devastating consequences of the widespread use and abuse of alcohol. Prenatal ethanol exposure is considered to be the leading known non-genetic cause of mental retardation in the Western world. Growing evidence suggests a major contribution of apoptosis to ethanol-induced teratogenesis. This discovery suggests that therapeutic strategies directed against apoptosis are particularly valuable for the prevention of FASD. However, there is a fundamental gap in understanding how ethanol leads to apoptosis in embryos. The continued existence of this gap represents an important problem because, until it is filled, understanding of ethanol-induced apoptosis that leads to teratogenesis will remain largely incomprehensible.

Sulforaphane (SFN) is a chemical that is abundant in broccoli sprouts. It was recently discovered that SFN can regulate gene expression through epigenetic mechanisms, specifically by decreasing the activities of histone deacetylase and DNA methyltransferase. This supports the potential of dietary consumption of SFN 
or SFN-rich broccoli sprouts to attenuate ethanol-induced apoptosis and confer in vivo protection against FASD through epigenetic regulation of the expression of anti-apoptotic genes. The objective of the work outlined in this study is to elucidate the epigenetic mechanisms by which ethanol induces apoptosis and teratogenesis and SFN regulates the anti-apoptotic gene expression and prevents ethanolinduced apoptosis, and to develop a safe and effective regimen to diminish the incidence and severity of FASD using SFN or SFN-rich broccoli sprouts, a safe "nutraceutical".

Our studies have demonstrated that SFN protects against ethanol-induced apoptosis in hNCCs through diminishing ethanol-induced hypermethylation at the promoters of the anti-apoptotic genes. SFN can also prevent hNCCs from ethanolinduced apoptosis by diminishing ethanol-induced reduction of histone acetylation at the promoters of the anti-apoptotic genes. In addition, SFN protects against ethanol-induced apoptosis in NCCs and zebrafish embryos through modulating histone methylation and the expression of Snail1 and restoring EMT. Moreover, our studies have shown that maternal dietary administration of SFN-rich BSE diminished ethanol-induced apoptosis and teratogenesis in mouse embryos by epigenetically modulating anti-apoptotic genes. These results elucidate that epigenetic silencing of the anti-apoptotic genes through promoter hypermethylation and histone deacetylation is involved in ethanol-induced apoptosis and teratogenesis and that SFN can prevent ethanol-induced apoptosis and teratogenesis by preventing the aberrant epigenetic modifications. 
These findings are significant because a better understanding of the epigenetic mechanisms underlying ethanol-induced apoptosis is a necessary prerequisite to the development of strategies against ethanol-induced teratogenesis. The insights gained from this study help to define the role of SFN and broccoli sprout extract (BSE), a diet-derived epigenetic modulator, in the prevention of FASD. These results may establish dietary supplementation of broccoli sprout extract as a novel strategy for the prevention of human FASD, providing high translational relevance. Once such highly translatable strategies become available, important advances in the intervention of FASD could be expected. Regarding its impact on scientific advancement, the findings from this study not only advance the field of FASD research but also contribute to a broader understanding of the epigenetic regulation of apoptosis.

\section{Strengths and limitations}

\section{Strengths}

There are a number of strengths of the research work presented in this dissertation. First, this is an innovative research work because it focuses on a novel approach, targeting epigenetic modulation directly involved in the apoptotic pathway, to prevent ethanol-induced apoptosis and teratogenesis. The theoretical concept described in the application is highly innovative because this is the first study attempting to prevent ethanol-induced apoptosis and teratogenesis specifically through the newly recognized actions of SFN or broccoli sprout extract as a dietary epigenetic regulator. Second, the approaches used in this research work are also innovative. These studies combine new and exciting discoveries in 
epigenetics with state-of-the-art approaches, including quantitative real-time PCR, siRNA knockdown technology and ChIP-PCR approach. Third, this research uniquely integrates in vitro cell culture and in vivo mouse and zebrafish model systems to elucidate the mechanisms by which ethanol induces apoptosis and teratogenesis and SFN prevents ethanol-induced apoptosis and teratogenesis. Fourth, these studies combine epigenetic analysis and morphological characterization techniques to assess the role of SFN in modulating ethanolinduced teratogenesis. Fifth, this is the first study using human neural crest cells (hNCCs) derived from human embryonic stem cells (hESCs) to elucidate the mechanisms underlying FASD. The use of hNCCs in this study is more closely mimic human responses in comparison to rodent cell lines and generates more relevant data representing human FASD, and therefore, has high translational potential for preventing FASD. Sixth, this research work represents the first attempt to prevent FASD through the use of bioactive compounds derived from a vegetable. The use of safe and effective epigenetic aberration-neutralizing dietary broccoli sprout extract is not only innovative but also has high translational potential for preventing FASD.

\section{Limitations}

There are also several limitations of the research work presented in this dissertation. One of the limitations is the siRNA technique that has been used to knock down the genes. It is well known that one of the major concerns for the siRNA approach is off-target effects. For the siRNA experiments in Chapter III, IV and $V, I$ have used the siRNA pool, a mixture of 4 siRNAs, to target the genes of 
interest. Evidence has shown that the use of pooling of multiple siRNAs to target gene is an effective way to reduce off-target silencing because of competition among the siRNA in the pool. In addition, since all members of the pool silence the same gene but have unique off-target signatures, this approach provides a selective reduction of off-target effects ${ }^{245}, 246$. However, while the pooling of multiple siRNAs can significantly reduce the off-target effects, it is impossible for this approach to completely address the concern with the off-target effects of the siRNA experiment. To further address this concern, a second siRNA pool might be needed to validate the results from the studies using siRNA. In addition to the siRNA technique, methylated specific PCR (MSP) that has been used to determine the methylation at the promoter regions of the anti-apoptotic genes in Chapter III and Chapter VI is another limitation. Although MSP is a highly effective protocol to detect the DNA methylation status, it cannot quantify the exact methylated or unmethylated cytosine because the products the PCR amplification was examined on an electrophoresis gel. For this reason, follow-up studies are needed to confirm specific cytosines that were methylated by using pyrosequencing, a protocol that can accurately measure the degree of methylation at each CpGs with high quantitative resolution.

\section{E. Future Directions}

While the findings from the study s described in this dissertation provide new insight into the epigenetic mechanisms underlying ethanol-induced apoptosis and teratogenesis, this work also provides a foundation for our future studies, as outlined in this section. 


\section{Elucidation of the molecular mechanisms underlying ethanol-induced}

increase in the activity of DNMTs and HDACs and up-regulation of DNMT3a, HDAC2 and KDM5A.

Our present study has shown that ethanol exposure can increase the activity of DNMTs and the expression of DNMT3a in hNCCs. Ethanol treatment also resulted in an increase in the activity of HDACs and the up-regulation of HDAC2. In addition, exposure to ethanol significantly increased the expression of KDM5A in hNCCs. Ethanol-induced increases in the activities of DNMTs and HDACs and the upregulation of DNMT3a and HDAC2 contribute to the hypermethylation and reduction of the histone acetylation at the promoter regions of the anti-apoptotic genes, the repression of anti-apoptotic genes and apoptosis in hNCCs. Upregulation of KDM5A also resulted in the reduction of $\mathrm{H} 3 \mathrm{~K} 4 \mathrm{me} 3$ enrichment at the promoters of Snail1, leading to the inhibition of EMT and apoptosis in hNCCs. However, the mechanisms by which ethanol-induced increases in the activities of DNMTs and HDACs, as well as the up-regulation of DNMT3a, HDAC2 and KDM5A are not clear and could be a very interesting future direction. Elucidation of the mechanisms underlying ethanol-induced increases in the activities of DNMTs and HDACs and the up-regulation of DNMT3a, HDAC2 and KDM5A may identify additional targets for the prevention of ethanol-induced apoptosis and FASD.

\section{Determination of the synergistic pro-apoptotic effects of epigenetic modulators, DNMTs, HDACs and KDM in ethanol-exposed hNCCs.}

The results from our studies present in this dissertation have clearly shown that an increase in the activity of DNMTs and up-regulation of DNMT3a contribute to 
ethanol-induced apoptosis in hNCCs by resulting in hypermethylation at the promoters of the anti-apoptotic genes and repressing the expression of these genes. We have also shown that an increase in the activity of HDACs and upregulation of HDAC2 in ethanol-exposed hNCCs resulted in the reduction of the acetylation at the promoter regions of the anti-apoptotic genes, which also leads to apoptosis. In addition, ethanol-induced up-regulation of KDM5A also contributes to ethanol-induced apoptosis by modulating the histone methylation at the promoters of Snail1. However, how these epigenetic modulators work together to induce apoptosis in ethanol-exposed hNCCs is not clear. Determination of the synergistic pro-apoptotic effects of DNMTs, HDACs and KDMs would be another important future direction.

\section{F. Summary and conclusions}

FASD is among the most devastating consequences of the widespread use and abuse of alcohol. Growing evidences suggest a major contribution of apoptosis to ethanol-induced teratogenesis. This discovery suggests that therapeutic strategies directed against apoptosis are particularly valuable for the prevention of FASD. Sulforaphane (SFN) is a chemical that is abundant in broccoli sprouts and can regulate gene expression through epigenetic mechanisms, specifically by decreasing the activities of histone deacetylase and DNA methyltransferase. The objective of this work is to elucidate the epigenetic mechanisms by which ethanol induces apoptosis and teratogenesis and SFN regulates the anti-apoptotic gene expression and prevents ethanol-induced apoptosis, and to develop a safe and effective regimen to diminish the incidence 
and severity of FASD using SFN or SFN-rich broccoli sprouts. Our studies have demonstrated that SFN protects against ethanol-induced apoptosis in hNCCs through diminishing ethanol-induced hypermethylation at the promoters of the antiapoptotic genes. SFN can also prevent hNCCs from ethanol-induced apoptosis by diminishing ethanol-induced reduction of histone acetylation at the promoters of the anti-apoptotic genes. In addition, SFN protects against ethanol-induced apoptosis in NCCs and zebrafish embryos through modulating histone methylation and the expression of Snail1 and restoring EMT. Moreover, our studies have shown that maternal dietary administration of SFN-rich BSE diminished ethanolinduced apoptosis and teratogenesis in mouse embryos by epigenetically modulating anti-apoptotic genes. These results elucidate that epigenetic silencing of the anti-apoptotic genes through promoter hypermethylation and histone deacetylation is involved in ethanol-induced apoptosis and teratogenesis and that SFN can prevent ethanol-induced apoptosis and teratogenesis by preventing the aberrant epigenetic modifications. These findings provide new insight into the epigenetic mechanisms underlying ethanol-induced apoptosis, which help to define the role of SFN and broccoli sprout extract, a diet-derived epigenetic modulator, in the prevention of FASD. These results may establish dietary supplementation of broccoli sprout extract as a novel strategy for the prevention of human FASD. 


\section{REFERENCES}

1. Lange, S. et al. Global Prevalence of Fetal Alcohol Spectrum Disorder Among Children and Youth: A Systematic Review and Meta-analysis. JAMA Pediatr 171, 948-956 (2017).

2. May, P.A. et al. Prevalence and characteristics of fetal alcohol spectrum disorders. Pediatrics 134, 855-866 (2014).

3. Sulik, K.K. \& Johnston, M.C. Sequence of developmental alterations following acute ethanol exposure in mice: craniofacial features of the fetal alcohol syndrome. Am J Anat 166, 257-269 (1983).

4. Sulik, K.K., Cook, C.S. \& Webster, W.S. Teratogens and craniofacial malformations: relationships to cell death. Development 103 Suppl, 213231 (1988).

5. Parnell, S.E. et al. Magnetic resonance microscopy defines ethanolinduced brain abnormalities in prenatal mice: effects of acute insult on gestational day 8. Alcohol Clin Exp Res 33, 1001-1011 (2009).

6. Godin, E.A. et al. Magnetic resonance microscopy defines ethanolinduced brain abnormalities in prenatal mice: effects of acute insult on gestational day 7. Alcohol Clin Exp Res 34, 98-111 (2010).

7. Lipinski, R.J. et al. Ethanol-induced face-brain dysmorphology patterns are correlative and exposure-stage dependent. PLoS One 7, e43067 (2012).

8. Chen, S.Y., Yang, B., Jacobson, K. \& Sulik, K.K. The membrane disordering effect of ethanol on neural crest cells in vitro and the protective role of GM1 ganglioside. Alcohol 13, 589-595 (1996).

9. Etchevers, H.C., Amiel, J. \& Lyonnet, S. Molecular bases of human neurocristopathies. Adv Exp Med Biol 589, 213-234 (2006).

10. Huang, X. \& Saint-Jeannet, J.P. Induction of the neural crest and the opportunities of life on the edge. Dev Biol 275, 1-11 (2004).

11. Graham, A., Begbie, J. \& McGonnell, I. Significance of the cranial neural crest. Dev Dyn 229, 5-13 (2004).

12. Creuzet, S., Couly, G. \& Le Douarin, N.M. Patterning the neural crest derivatives during development of the vertebrate head: insights from avian studies. J Anat 207, 447-459 (2005).

13. Minoux, M. \& Rijli, F.M. Molecular mechanisms of cranial neural crest cell migration and patterning in craniofacial development. Development 137, 2605-2621 (2010).

14. Kotch, L.E. \& Sulik, K.K. Patterns of ethanol-induced cell death in the developing nervous system of mice; neural fold states through the time of anterior neural tube closure. Int J Dev Neurosci 10, $273-279$ (1992). 
15. Rovasio, R.A. \& Battiato, N.L. Ethanol induces morphological and dynamic changes on in vivo and in vitro neural crest cells. Alcohol Clin Exp Res 26, 1286-1298 (2002).

16. Flentke, G.R., Garic, A., Amberger, E., Hernandez, M. \& Smith, S.M. Calcium-mediated repression of beta-catenin and its transcriptional signaling mediates neural crest cell death in an avian model of fetal alcohol syndrome. Birth Defects Res A Clin Mol Teratol 91, 591-602 (2011).

17. Chen, X., Liu, J. \& Chen, S.Y. Sulforaphane protects against ethanolinduced oxidative stress and apoptosis in neural crest cells by the induction of Nrf2-mediated antioxidant response. Br J Pharmacol 169, 437-448 (2013).

18. Yuan, F. et al. Sulforaphane restores acetyl-histone $\mathrm{H} 3$ binding to $\mathrm{Bcl}-2$ promoter and prevents apoptosis in ethanol-exposed neural crest cells and mouse embryos. Exp Neurol 300, 60-66 (2018).

19. Chen, X., Liu, J. \& Chen, S.Y. Over-expression of Nrf2 diminishes ethanolinduced oxidative stress and apoptosis in neural crest cells by inducing an antioxidant response. Reprod Toxicol 42, 102-109 (2013).

20. Liu, Y., Balaraman, Y., Wang, G., Nephew, K.P. \& Zhou, F.C. Alcohol exposure alters DNA methylation profiles in mouse embryos at early neurulation. Epigenetics 4, 500-511 (2009).

21. Kobor, M.S. \& Weinberg, J. Focus on: epigenetics and fetal alcohol spectrum disorders. Alcohol Res Health 34, 29-37 (2011).

22. Ouko, L.A. et al. Effect of alcohol consumption on CpG methylation in the differentially methylated regions of $\mathrm{H} 19$ and IG-DMR in male gametes: implications for fetal alcohol spectrum disorders. Alcohol Clin Exp Res 33, 1615-1627 (2009).

23. Zhou, F.C. et al. Alcohol alters DNA methylation patterns and inhibits neural stem cell differentiation. Alcohol Clin Exp Res 35, 735-746 (2011).

24. Haycock, P.C. \& Ramsay, M. Exposure of mouse embryos to ethanol during preimplantation development: effect on DNA methylation in the h19 imprinting control region. Biol Reprod 81, 618-627 (2009).

25. Subbanna, S., Nagre, N.N., Umapathy, N.S., Pace, B.S. \& Basavarajappa, B.S. Ethanol exposure induces neonatal neurodegeneration by enhancing CB1R Exon1 histone H4K8 acetylation and up-regulating CB1R function causing neurobehavioral abnormalities in adult mice. Int $\mathrm{J}$ Neuropsychopharmacol 18 (2014).

26. Subbanna, S. et al. Ethanol induced acetylation of histone at G9a exon1 and G9a-mediated histone $\mathrm{H} 3$ dimethylation leads to neurodegeneration in neonatal mice. Neuroscience 258, $422-432$ (2014).

27. Subbanna, S. et al. G9a-mediated histone methylation regulates ethanolinduced neurodegeneration in the neonatal mouse brain. Neurobiol Dis 54, 475-485 (2013).

28. Bekdash, R.A., Zhang, C. \& Sarkar, D.K. Gestational choline supplementation normalized fetal alcohol-induced alterations in histone 
modifications, DNA methylation, and proopiomelanocortin (POMC) gene expression in beta-endorphin-producing POMC neurons of the hypothalamus. Alcohol Clin Exp Res 37, 1133-1142 (2013).

29. Zhong, L. et al. Ethanol and its metabolites induce histone lysine 9 acetylation and an alteration of the expression of heart developmentrelated genes in cardiac progenitor cells. Cardiovasc Toxicol 10, 268-274 (2010).

30. Garro, A.J., McBeth, D.L., Lima, V. \& Lieber, C.S. Ethanol consumption inhibits fetal DNA methylation in mice: implications for the fetal alcohol syndrome. Alcohol Clin Exp Res 15, 395-398 (1991).

31. Kaminen-Ahola, N. et al. Maternal ethanol consumption alters the epigenotype and the phenotype of offspring in a mouse model. Plos Genet 6, e1000811 (2010).

32. Zhou, F.C. DNA methylation program during development. Front Biol (Beijing) 7, 485-494 (2012).

33. Zhou, F.C., Sari, Y., Powrozek, T.A. \& Spong, C.Y. A neuroprotective peptide antagonizes fetal alcohol exposure-compromised brain growth. $J$ Mol Neurosci 24, 189-199 (2004).

34. Park, P.H., Miller, R. \& Shukla, S.D. Acetylation of histone H3 at lysine 9 by ethanol in rat hepatocytes. Biochem Bioph Res Co 306, 501-504 (2003).

35. Basavarajappa, B.S. \& Subbanna, S. Epigenetic Mechanisms in Developmental Alcohol-Induced Neurobehavioral Deficits. Brain Sci 6 (2016).

36. Basavarajappa, B.S. \& Subbanna, S. Epigenetic Mechanisms in Developmental Alcohol-Induced Neurobehavioral Deficits. Brain Sci 6 (2016).

37. Mathers, J.C., Strathdee, G. \& Relton, C.L. Induction of epigenetic alterations by dietary and other environmental factors. Adv Genet 71, 3-39 (2010).

38. Jones, P.A. \& Liang, G. Rethinking how DNA methylation patterns are maintained. Nat Rev Genet 10, 805-811 (2009).

39. Hervouet, E., Cheray, M., Vallette, F.M. \& Cartron, P.F. DNA methylation and apoptosis resistance in cancer cells. Cells 2, 545-573 (2013).

40. Gopisetty, G., Ramachandran, K. \& Singal, R. DNA methylation and apoptosis. Mol Immunol 43, 1729-1740 (2006).

41. Pompeia, C. et al. Microarray analysis of epigenetic silencing of gene expression in the KAS-6/1 multiple myeloma cell line. Cancer Res 64, 3465-3473 (2004).

42. Wu, J. \& Wood, G.S. Reduction of Fas/CD95 promoter methylation, upregulation of Fas protein, and enhancement of sensitivity to apoptosis in cutaneous T-cell lymphoma. Arch Dermatol 147, $443-449$ (2011).

43. Chaopatchayakul, P., Jearanaikoon, P., Yuenyao, P. \& Limpaiboon, T. Aberrant DNA methylation of apoptotic signaling genes in patients responsive and nonresponsive to therapy for cervical carcinoma. Am J Obstet Gynecol 202, 281 e281-289 (2010). 
44. Petak, I. et al. Hypermethylation of the gene promoter and enhancer region can regulate Fas expression and sensitivity in colon carcinoma. Cell Death Differ 10, 211-217 (2003).

45. Okami, J., Simeone, D.M. \& Logsdon, C.D. Silencing of the hypoxiainducible cell death protein BNIP3 in pancreatic cancer. Cancer Res 64, 5338-5346 (2004).

46. Hervouet, E., Vallette, F.M. \& Cartron, P.F. Impact of the DNA methyltransferases expression on the methylation status of apoptosisassociated genes in glioblastoma multiforme. Cell Death Dis 1, e8 (2010).

47. Lyu, H., Huang, J., He, Z. \& Liu, B. Epigenetic mechanism of survivin dysregulation in human cancer. Sci China Life Sci 61, 808-814 (2018).

48. Kouzarides, T. Chromatin modifications and their function. Cell 128, 693705 (2007).

49. Javaid, N. \& Choi, S. Acetylation- and Methylation-Related Epigenetic Proteins in the Context of Their Targets. Genes (Basel) 8 (2017).

50. Liang, G. \& Weisenberger, D.J. DNA methylation aberrancies as a guide for surveillance and treatment of human cancers. Epigenetics 12, 416-432 (2017).

51. Jones, P.A., Issa, J.P. \& Baylin, S. Targeting the cancer epigenome for therapy. Nat Rev Genet 17, 630-641 (2016).

52. Yamaguchi, M. et al. Histone deacetylase 1 regulates retinal neurogenesis in zebrafish by suppressing Wnt and Notch signaling pathways. Development 132, 3027-3043 (2005).

53. Lagger, G. et al. Essential function of histone deacetylase 1 in proliferation control and CDK inhibitor repression. EMBO J 21, 2672-2681 (2002).

54. Brunmeir, R., Lagger, S. \& Seiser, C. Histone deacetylase HDAC1/HDAC2-controlled embryonic development and cell differentiation. Int J Dev Biol 53, 275-289 (2009).

55. Seto, E. \& Yoshida, M. Erasers of histone acetylation: the histone deacetylase enzymes. Cold Spring Harb Perspect Biol 6, a018713 (2014).

56. Peserico, A. \& Simone, C. Physical and functional HAT/HDAC interplay regulates protein acetylation balance. J Biomed Biotechnol 2011, 371832 (2011).

57. Giannini, G., Cabri, W., Fattorusso, C. \& Rodriquez, M. Histone deacetylase inhibitors in the treatment of cancer: overview and perspectives. Future Med Chem 4, 1439-1460 (2012).

58. Zhang, $\mathrm{H}$. et al. Histone Deacetylases Inhibitors in the Treatment of Retinal Degenerative Diseases: Overview and Perspectives. J Ophthalmol 2015, 250812 (2015).

59. Chuang, M.J. et al. The HDAC inhibitor LBH589 induces ERK-dependent prometaphase arrest in prostate cancer via HDAC6 inactivation and downregulation. PLoS One 8, e73401 (2013).

60. Feng, W., Zhang, B., Cai, D. \& Zou, X. Therapeutic potential of histone deacetylase inhibitors in pancreatic cancer. Cancer Lett 347, 183-190 (2014). 
61. Khabele, D. The therapeutic potential of class I selective histone deacetylase inhibitors in ovarian cancer. Front Oncol 4, 111 (2014).

62. Salvador, M.A. et al. The histone deacetylase inhibitor abexinostat induces cancer stem cells differentiation in breast cancer with low Xist expression. Clin Cancer Res 19, 6520-6531 (2013).

63. Mariadason, J.M. HDACs and HDAC inhibitors in colon cancer. Epigenetics 3, 28-37 (2008).

64. Ren, M., Leng, Y., Jeong, M., Leeds, P.R. \& Chuang, D.M. Valproic acid reduces brain damage induced by transient focal cerebral ischemia in rats: potential roles of histone deacetylase inhibition and heat shock protein induction. J Neurochem 89, 1358-1367 (2004).

65. Kim, H.J. et al. Histone deacetylase inhibitors exhibit anti-inflammatory and neuroprotective effects in a rat permanent ischemic model of stroke: multiple mechanisms of action. J Pharmacol Exp Ther 321, 892-901 (2007).

66. Chuang, D.M., Leng, Y., Marinova, Z., Kim, H.J. \& Chiu, C.T. Multiple roles of HDAC inhibition in neurodegenerative conditions. Trends Neurosci 32, 591-601 (2009).

67. Ferrante, R.J. et al. Histone deacetylase inhibition by sodium butyrate chemotherapy ameliorates the neurodegenerative phenotype in Huntington's disease mice. J Neurosci 23, 9418-9427 (2003).

68. Zhang, Z. et al. Valproic acid-mediated neuroprotection in retinal ischemia injury via histone deacetylase inhibition and transcriptional activation. Exp Eye Res 94, 98-108 (2012).

69. Kim, H.J., Leeds, P. \& Chuang, D.M. The HDAC inhibitor, sodium butyrate, stimulates neurogenesis in the ischemic brain. $J$ Neurochem 110, 1226-1240 (2009).

70. Sinn, D.I. et al. Valproic acid-mediated neuroprotection in intracerebral hemorrhage via histone deacetylase inhibition and transcriptional activation. Neurobiol Dis 26, 464-472 (2007).

71. Zhang, Z.Z. et al. Valproate promotes survival of retinal ganglion cells in a rat model of optic nerve crush. Neuroscience 224, 282-293 (2012).

72. Zhang, T., Cooper, S. \& Brockdorff, N. The interplay of histone modifications - writers that read. EMBO Rep 16, 1467-1481 (2015).

73. Turner, B.M. Cellular memory and the histone code. Cell 111, 285-291 (2002).

74. Aday, A.W., Zhu, L.J., Lakshmanan, A., Wang, J. \& Lawson, N.D. Identification of cis regulatory features in the embryonic zebrafish genome through large-scale profiling of $\mathrm{H} 3 \mathrm{~K} 4 \mathrm{me} 1$ and $\mathrm{H} 3 \mathrm{~K} 4 \mathrm{me} 3$ binding sites. Dev Biol 357, 450-462 (2011).

75. $\mathrm{Bi}, \mathrm{Y}$. et al. WDR82, a key epigenetics-related factor, plays a crucial role in normal early embryonic development in mice. Biol Reprod 84, 756-764 (2011).

76. Pan, G. et al. Whole-genome analysis of histone H3 lysine 4 and lysine 27 methylation in human embryonic stem cells. Cell Stem Cell 1, 299-312 (2007). 
77. Szutorisz, H. \& Dillon, N. The epigenetic basis for embryonic stem cell pluripotency. Bioessays 27, 1286-1293 (2005).

78. Cao, J. et al. Histone demethylase RBP2 is critical for breast cancer progression and metastasis. Cell Rep 6, 868-877 (2014).

79. Hou, J. et al. Genomic amplification and a role in drug-resistance for the KDM5A histone demethylase in breast cancer. Am J Transl Res 4, 247256 (2012).

80. Klose, R.J. et al. The retinoblastoma binding protein RBP2 is an H3K4 demethylase. Cell 128, 889-900 (2007).

81. Nan, X. et al. Transcriptional repression by the methyl-CpG-binding protein MeCP2 involves a histone deacetylase complex. Nature 393, 386389 (1998).

82. Parry, L. \& Clarke, A.R. The Roles of the Methyl-CpG Binding Proteins in Cancer. Genes Cancer 2, 618-630 (2011).

83. Ben-Porath, I. \& Cedar, H. Epigenetic crosstalk. Mol Cell 8, 933-935 (2001).

84. Fuks, F., Hurd, P.J., Deplus, R. \& Kouzarides, T. The DNA methyltransferases associate with HP1 and the SUV39H1 histone methyltransferase. Nucleic Acids Res 31, 2305-2312 (2003).

85. Fujita, N. et al. Methyl-CpG binding domain 1 (MBD1) interacts with the Suv39h1-HP1 heterochromatic complex for DNA methylation-based transcriptional repression. J Biol Chem 278, 24132-24138 (2003).

86. Hakimi, M.A. et al. A core-BRAF35 complex containing histone deacetylase mediates repression of neuronal-specific genes. Proc Natl Acad Sci U S A 99, 7420-7425 (2002).

87. Humphrey, G.W. et al. Stable histone deacetylase complexes distinguished by the presence of SANT domain proteins CoREST/kiaa0071 and Mta-L1. J Biol Chem 276, 6817-6824 (2001).

88. Song, M.J. et al. Epigenome mapping highlights chromatin-mediated gene regulation in the protozoan parasite Trichomonas vaginalis. Sci Rep 7, 45365 (2017).

89. Huang, Y., Vasilatos, S.N., Boric, L., Shaw, P.G. \& Davidson, N.E. Inhibitors of histone demethylation and histone deacetylation cooperate in regulating gene expression and inhibiting growth in human breast cancer cells. Breast Cancer Res Treat 131, 777-789 (2012).

90. Singh, M.M. et al. Inhibition of LSD1 sensitizes glioblastoma cells to histone deacetylase inhibitors. Neuro Oncol 13, 894-903 (2011).

91. Theveneau, E. \& Mayor, R. Neural crest delamination and migration: from epithelium-to-mesenchyme transition to collective cell migration. Dev Biol 366, 34-54 (2012).

92. Zhang, D. et al. The neural crest: a versatile organ system. Birth Defects Res C Embryo Today 102, 275-298 (2014).

93. Maurer, J. et al. Establishment and controlled differentiation of neural crest stem cell lines using conditional transgenesis. Differentiation 75, 580-591 (2007).

94. Shakhova, O. \& Sommer, L. in StemBook (Cambridge (MA); 2008). 
95. Kalluri, R. \& Weinberg, R.A. The basics of epithelial-mesenchymal transition. J Clin Invest 119, 1420-1428 (2009).

96. Thiery, J.P., Acloque, H., Huang, R.Y. \& Nieto, M.A. Epithelialmesenchymal transitions in development and disease. Cell 139, 871-890 (2009).

97. Barriere, G., Tartary, M. \& Rigaud, M. Epithelial mesenchymal transition: a new insight into the detection of circulating tumor cells. ISRN Oncol 2012, 382010 (2012).

98. Nieto, M.A., Huang, R.Y., Jackson, R.A. \& Thiery, J.P. Emt: 2016. Cell 166, 21-45 (2016).

99. Powell, D.R., Blasky, A.J., Britt, S.G. \& Artinger, K.B. Riding the crest of the wave: parallels between the neural crest and cancer in epithelial-tomesenchymal transition and migration. Wiley Interdiscip Rev Syst Biol Med 5, 511-522 (2013).

100. Heldin, C.H., Landstrom, M. \& Moustakas, A. Mechanism of TGF-beta signaling to growth arrest, apoptosis, and epithelial-mesenchymal transition. Curr Opin Cell Biol 21, 166-176 (2009).

101. Liu, Y. et al. YAP modulates TGF-beta1-induced simultaneous apoptosis and EMT through upregulation of the EGF receptor. Sci Rep 7, 45523 (2017).

102. Robson, E.J., Khaled, W.T., Abell, K. \& Watson, C.J. Epithelial-tomesenchymal transition confers resistance to apoptosis in three murine mammary epithelial cell lines. Differentiation 74, 254-264 (2006).

103. Park, S.J. et al. Induction of Apoptosis and Inhibition of Epithelial Mesenchymal Transition by alpha-Mangostin in MG-63 Cell Lines. Evid Based Complement Alternat Med 2018, 3985082 (2018).

104. Cano, A. et al. The transcription factor snail controls epithelialmesenchymal transitions by repressing E-cadherin expression. Nat Cell Biol 2, 76-83 (2000).

105. Kaufhold, S. \& Bonavida, B. Central role of Snail1 in the regulation of EMT and resistance in cancer: a target for therapeutic intervention. J Exp Clin Cancer Res 33, 62 (2014).

106. Lin, Y., Dong, C. \& Zhou, B.P. Epigenetic regulation of EMT: the Snail story. Curr Pharm Des 20, 1698-1705 (2014).

107. Batlle, E. et al. The transcription factor snail is a repressor of E-cadherin gene expression in epithelial tumour cells. Nat Cell Biol 2, 84-89 (2000).

108. Feng, J. et al. Histone deacetylase inhibitor valproic acid (VPA) promotes the epithelial mesenchymal transition of colorectal cancer cells via up regulation of Snail. Cell Adh Migr 9, 495-501 (2015).

109. Ji, M. et al. HDAC inhibitors induce epithelial-mesenchymal transition in colon carcinoma cells. Oncol Rep 33, 2299-2308 (2015).

110. Giudice, F.S., Pinto, D.S., Jr., Nor, J.E., Squarize, C.H. \& Castilho, R.M. Inhibition of histone deacetylase impacts cancer stem cells and induces epithelial-mesenchyme transition of head and neck cancer. PLoS One 8, e58672 (2013). 
111. $\mathrm{Li}$, D. et al. Role of RbBP5 and H3K4me3 in the vicinity of Snail transcription start site during epithelial-mesenchymal transition in prostate cancer cell. Oncotarget 7, 65553-65567 (2016).

112. Do, D.P., Pai, S.B., Rizvi, S.A. \& D'Souza, M.J. Development of sulforaphane-encapsulated microspheres for cancer epigenetic therapy. Int J Pharm 386, 114-121 (2010).

113. Bai, Y. et al. Prevention by sulforaphane of diabetic cardiomyopathy is associated with up-regulation of Nrf2 expression and transcription activation. J Mol Cell Cardiol 57, 82-95 (2013).

114. Cui, W. et al. Prevention of diabetic nephropathy by sulforaphane: possible role of Nrf2 upregulation and activation. Oxid Med Cell Longev 2012, 821936 (2012).

115. Su, X. et al. Anticancer Activity of Sulforaphane: The Epigenetic Mechanisms and the Nrf2 Signaling Pathway. Oxid Med Cell Longev 2018, 5438179 (2018).

116. Ali Khan, M. et al. Sulforaphane Reverses the Expression of Various Tumor Suppressor Genes by Targeting DNMT3B and HDAC1 in Human Cervical Cancer Cells. Evid Based Complement Alternat Med 2015, 412149 (2015).

117. Fan, H. et al. Sulforaphane causes a major epigenetic repression of myostatin in porcine satellite cells. Epigenetics 7, 1379-1390 (2012).

118. Qu, X. et al. Sulforaphane epigenetically regulates innate immune responses of porcine monocyte-derived dendritic cells induced with lipopolysaccharide. PLoS One 10, e0121574 (2015).

119. Atwell, L.L. et al. Absorption and chemopreventive targets of sulforaphane in humans following consumption of broccoli sprouts or a myrosinasetreated broccoli sprout extract. Mol Nutr Food Res 59, 424-433 (2015).

120. Singh, K. et al. Sulforaphane treatment of autism spectrum disorder (ASD). Proc Natl Acad Sci U S A 111, 15550-15555 (2014).

121. Tortorella, S.M., Royce, S.G., Licciardi, P.V. \& Karagiannis, T.C. Dietary Sulforaphane in Cancer Chemoprevention: The Role of Epigenetic Regulation and HDAC Inhibition. Antioxid Redox Signal 22, 1382-1424 (2015).

122. Benedict, A.L. et al. Neuroprotective effects of sulforaphane after contusive spinal cord injury. J Neurotrauma 29, 2576-2586 (2012).

123. Abbaoui, B. et al. Inhibition of bladder cancer by broccoli isothiocyanates sulforaphane and erucin: characterization, metabolism, and interconversion. Mol Nutr Food Res 56, 1675-1687 (2012).

124. Tang, L. et al. Potent activation of mitochondria-mediated apoptosis and arrest in $\mathrm{S}$ and $\mathrm{M}$ phases of cancer cells by a broccoli sprout extract. Mol Cancer Ther 5, 935-944 (2006).

125. Myzak, M.C., Tong, P., Dashwood, W.M., Dashwood, R.H. \& Ho, E. Sulforaphane retards the growth of human PC-3 xenografts and inhibits HDAC activity in human subjects. Exp Biol Med (Maywood) 232, 227-234 (2007). 
126. Shirai, Y. et al. Dietary Intake of Sulforaphane-Rich Broccoli Sprout Extracts during Juvenile and Adolescence Can Prevent PhencyclidineInduced Cognitive Deficits at Adulthood. PLoS One 10, e0127244 (2015).

127. Westerfield, M. The zebrafish book : a guide for the laboratory use of zebrafish (Danio rerio), Edn. 5th Edition. (Printed by the University of Oregon Press, Eugene, OR; 2007).

128. Sokol, R.J., Delaney-Black, V. \& Nordstrom, B. Fetal alcohol spectrum disorder. JAMA 290, 2996-2999 (2003).

129. Koren, G., Nulman, I., Chudley, A.E. \& Loocke, C. Fetal alcohol spectrum disorder. CMAJ 169, 1181-1185 (2003).

130. Dunty, W.C., Jr., Chen, S.Y., Zucker, R.M., Dehart, D.B. \& Sulik, K.K. Selective vulnerability of embryonic cell populations to ethanol-induced apoptosis: implications for alcohol-related birth defects and neurodevelopmental disorder. Alcohol Clin Exp Res 25, 1523-1535 (2001).

131. Cartwright, M.M. \& Smith, S.M. Increased cell death and reduced neural crest cell numbers in ethanol-exposed embryos: partial basis for the fetal alcohol syndrome phenotype. Alcohol Clin Exp Res 19, 378-386 (1995).

132. Chen, X., Liu, J., Feng, W.K., Wu, X. \& Chen, S.Y. MiR-125b protects against ethanol-induced apoptosis in neural crest cells and mouse embryos by targeting Bak 1 and PUMA. Exp Neurol 271, 104-111 (2015).

133. $\mathrm{Li}, \mathrm{Y}$. et al. Sulforaphane protects against ethanol-induced apoptosis in neural crest cells through restoring epithelial-mesenchymal transition by epigenetically modulating the expression of Snail1. Biochim Biophys Acta Mol Basis Dis 1865, 2586-2594 (2019).

134. Jones, P.A. \& Baylin, S.B. The fundamental role of epigenetic events in cancer. Nat Rev Genet 3, 415-428 (2002).

135. Teodoridis, J.M., Strathdee, G. \& Brown, R. Epigenetic silencing mediated by $\mathrm{CpG}$ island methylation: potential as a therapeutic target and as a biomarker. Drug Resist Updat 7, 267-278 (2004).

136. Malekzadeh, K. et al. Methylation patterns of Rb1 and Casp-8 promoters and their impact on their expression in bladder cancer. Cancer Invest 27, 70-80 (2009).

137. Murphy, T.M., Perry, A.S. \& Lawler, M. The emergence of DNA methylation as a key modulator of aberrant cell death in prostate cancer. Endocr-Relat Cancer 15, 11-25 (2008).

138. Harada, K. et al. Deregulation of caspase 8 and 10 expression in pediatric tumors and cell lines. Cancer Res 62, 5897-5901 (2002).

139. Shivapurkar, N. et al. Differential inactivation of caspase-8 in lung cancers. Cancer Biol Ther 1, 65-69 (2002).

140. Wu, Y., Alvarez, M., Slamon, D.J., Koeffler, P. \& Vadgama, J.V. Caspase 8 and maspin are downregulated in breast cancer cells due to CpG site promoter methylation. BMC Cancer 10, 32 (2010).

141. Zou, Y. et al. DNA Hypermethylation of CREB3L1 and Bcl-2 Associated with the Mitochondrial-Mediated Apoptosis via PI3K/Akt Pathway in 
Human BEAS-2B Cells Exposure to Silica Nanoparticles. PLoS One 11, e0158475 (2016).

142. Singal, R. \& Ginder, G.D. DNA methylation. Blood 93, 4059-4070 (1999).

143. Jones, P.A. \& Liang, G.N. OPINION Rethinking how DNA methylation patterns are maintained. Nature Reviews Genetics 10, 805-811 (2009).

144. Chen, T., Ueda, Y., Dodge, J.E., Wang, Z. \& Li, E. Establishment and maintenance of genomic methylation patterns in mouse embryonic stem cells by Dnmt3a and Dnmt3b. Mol Cell Biol 23, 5594-5605 (2003).

145. Meeran, S.M., Patel, S.N., Li, Y., Shukla, S. \& Tollefsbol, T.O. Bioactive dietary supplements reactivate ER expression in ER-negative breast cancer cells by active chromatin modifications. PLoS One 7, e37748 (2012).

146. Qu, X.Q. et al. Sulforaphane Epigenetically Regulates Innate Immune Responses of Porcine Monocyte-Derived Dendritic Cells Induced with Lipopolysaccharide. Plos One 10 (2015).

147. Leonhardt, H. \& Bestor, T.H. Structure, function and regulation of mammalian DNA methyltransferase. EXS 64, 109-119 (1993).

148. Zahnow, C.A. et al. Inhibitors of DNA Methylation, Histone Deacetylation, and Histone Demethylation: A Perfect Combination for Cancer Therapy. Adv Cancer Res 130, 55-111 (2016).

149. Weissman, J., Naidu, S. \& Bjornsson, H.T. Abnormalities of the DNA methylation mark and its machinery: an emerging cause of neurologic dysfunction. Semin Neurol 34, 249-257 (2014).

150. Jensen Pena, C., Monk, C. \& Champagne, F.A. Epigenetic effects of prenatal stress on 11 beta-hydroxysteroid dehydrogenase- 2 in the placenta and fetal brain. PLoS One 7, e39791 (2012).

151. Sakharkar, A.J. et al. Effects of acute ethanol exposure on anxiety measures and epigenetic modifiers in the extended amygdala of adolescent rats. Int J Neuropsychopharmacol 17, 2057-2067 (2014).

152. $\mathrm{Li}, \mathrm{J}$. et al. Temporal DNA methylation pattern and targeted therapy in colitis-associated cancer. Carcinogenesis (2019).

153. Wei, D., Yu, G. \& Zhao, Y. MicroRNA-30a-3p inhibits the progression of lung cancer via the PI3K/AKT by targeting DNA methyltransferase 3a. Onco Targets Ther 12, 7015-7024 (2019).

154. Jing, W. et al. DNMT3a promotes proliferation by activating the STAT3 signaling pathway and depressing apoptosis in pancreatic cancer. Cancer Manag Res 11, 6379-6396 (2019).

155. Sulik, K.K., Johnston, M.C., Daft, P.A., Russell, W.E. \& Dehart, D.B. Fetal alcohol syndrome and DiGeorge anomaly: critical ethanol exposure periods for craniofacial malformations as illustrated in an animal model. Am J Med Genet Supp/ 2, 97-112 (1986).

156. Cartwright, M.M. \& Smith, S.M. Stage-dependent effects of ethanol on cranial neural crest cell development: partial basis for the phenotypic variations observed in fetal alcohol syndrome. Alcohol Clin Exp Res 19, 1454-1462 (1995). 
157. Cartwright, M.M., Tessmer, L.L. \& Smith, S.M. Ethanol-induced neural crest apoptosis is coincident with their endogenous death, but is mechanistically distinct. Alcohol Clin Exp Res 22, 142-149 (1998).

158. Chen, S.Y. \& Sulik, K.K. Free radicals and ethanol-induced cytotoxicity in neural crest cells. Alcohol Clin Exp Res 20, 1071-1076 (1996).

159. Kim, J.S. \& Shukla, S.D. Histone h3 modifications in rat hepatic stellate cells by ethanol. Alcohol Alcohol 40, 367-372 (2005).

160. Park, P.H., Miller, R. \& Shukla, S.D. Acetylation of histone H3 at lysine 9 by ethanol in rat hepatocytes. Biochem Biophys Res Commun 306, 501504 (2003).

161. Petanjek, Z. \& Kostovic, I. Epigenetic regulation of fetal brain development and neurocognitive outcome. Proc Natl Acad Sci U S A 109, 11062-11063 (2012).

162. Chen, H., Tini, M. \& Evans, R.M. HATs on and beyond chromatin. Curr Opin Cell Biol 13, 218-224 (2001).

163. $\mathrm{Li}, \mathrm{J}$. et al. Arsenic trioxide promotes histone $\mathrm{H} 3$ phosphoacetylation at the chromatin of CASPASE-10 in acute promyelocytic leukemia cells. J Biol Chem 277, 49504-49510 (2002).

164. Yan, X. et al. Inhibition of histone acetylation by curcumin reduces alcoholinduced fetal cardiac apoptosis. J Biomed Sci 24, 1 (2017).

165. Jones, P.A., Issa, J.P.J. \& Baylin, S. Targeting the cancer epigenome for therapy. Nature Reviews Genetics 17, 630-641 (2016).

166. Ciafre, S. et al. How alcohol drinking affects our genes: an epigenetic point of view. Biochem Cell Biol 97, 345-356 (2019).

167. Chater-Diehl, E.J., Laufer, B.I. \& Singh, S.M. Changes to histone modifications following prenatal alcohol exposure: An emerging picture. Alcohol 60, 41-52 (2017).

168. Blander, G. \& Guarente, L. The Sir2 family of protein deacetylases. Annu Rev Biochem 73, 417-435 (2004).

169. Haberland, M., Montgomery, R.L. \& Olson, E.N. The many roles of histone deacetylases in development and physiology: implications for disease and therapy. Nature Reviews Genetics 10, 32-42 (2009).

170. Gupta, P., Kim, B., Kim, S.H. \& Srivastava, S.K. Molecular targets of isothiocyanates in cancer: recent advances. Mol Nutr Food Res 58, 16851707 (2014).

171. Chang, C.C., Hung, C.M., Yang, Y.R., Lee, M.J. \& Hsu, Y.C. Sulforaphane induced cell cycle arrest in the G2/M phase via the blockade of cyclin B1/CDC2 in human ovarian cancer cells. J Ovarian Res 6, 41 (2013).

172. Atwell, L.L. et al. Epigenetic Regulation by Sulforaphane: Opportunities for Breast and Prostate Cancer Chemoprevention. Curr Pharmacol Rep 1, 102-111 (2015).

173. Khan, M.A. et al. Sulforaphane Reverses the Expression of Various Tumor Suppressor Genes by Targeting DNMT3B and HDAC1 in Human Cervical Cancer Cells. Evid-Based Compl Alt (2015). 
174. Dashwood, R.H., Myzak, M.C. \& Ho, E. Dietary HDAC inhibitors: time to rethink weak ligands in cancer chemoprevention? Carcinogenesis 27, 344-349 (2006).

175. Jiang, L.L., Zhou, S.J., Zhang, X.M., Chen, H.Q. \& Liu, W. Sulforaphane suppresses in vitro and in vivo lung tumorigenesis through downregulation of HDAC activity. Biomed Pharmacother 78, 74-80 (2016).

176. Sakharkar, A.J. et al. A role for histone acetylation mechanisms in adolescent alcohol exposure-induced deficits in hippocampal brainderived neurotrophic factor expression and neurogenesis markers in adulthood. Brain Struct Funct 221, 4691-4703 (2016).

177. Yoshida, M., Kudo, N., Kosono, S. \& Ito, A. Chemical and structural biology of protein lysine deacetylases. Proc Jpn Acad Ser B Phys Biol Sci 93, 297-321 (2017).

178. Xiang, B. et al. Systematic genetic analyses of genome-wide association study data reveal an association between the key nucleosome remodeling and deacetylase complex and bipolar disorder development. Bipolar Disord 20, 370-380 (2018).

179. Lucia, D. et al. Periconceptional maternal alcohol consumption leads to behavioural changes in adult and aged offspring and alters the expression of hippocampal genes associated with learning and memory and regulators of the epigenome. Behav Brain Res 362, 249-257 (2019).

180. Agudelo, M. et al. Effects of alcohol on histone deacetylase 2 (HDAC2) and the neuroprotective role of trichostatin A (TSA). Alcohol Clin Exp Res 35, 1550-1556 (2011).

181. Sakharkar, A.J. et al. Effects of histone deacetylase inhibitors on amygdaloid histone acetylation and neuropeptide $\mathrm{Y}$ expression: a role in anxiety-like and alcohol-drinking behaviours. Int J Neuropsychopharmacol 17, 1207-1220 (2014).

182. Du, Y., Tang, G. \& Yuan, W. Suppression of HDAC2 by sodium butyrate alleviates apoptosis of kidney cells in db/db mice and HGinduced NRK52E cells. Int J Mol Med (2019).

183. Wang, Y. et al. Modulations of Histone Deacetylase 2 Offer a Protective Effect through the Mitochondrial Apoptosis Pathway in Acute Liver Failure. Oxid Med Cell Longev 2019, 8173016 (2019).

184. Caputo, C., Wood, E. \& Jabbour, L. Impact of fetal alcohol exposure on body systems: A systematic review. Birth Defects Res C Embryo Today 108, 174-180 (2016).

185. Del Campo, M. \& Jones, K.L. A review of the physical features of the fetal alcohol spectrum disorders. Eur J Med Genet 60, 55-64 (2017).

186. Chen, S.Y., Dehart, D.B. \& Sulik, K.K. Protection from ethanol-induced limb malformations by the superoxide dismutase/catalase mimetic, EUK134. FASEB journal : official publication of the Federation of American Societies for Experimental Biology 18, 1234-1236 (2004).

187. Sulik, K.K. Fetal alcohol spectrum disorder: pathogenesis and mechanisms. Handb Clin Neurol 125, 463-475 (2014). 
188. Granato, A. \& Dering, B. Alcohol and the Developing Brain: Why Neurons Die and How Survivors Change. Int J Mol Sci 19 (2018).

189. Smith, S.M. Alcohol-induced cell death in the embryo. Alcohol Health Res World 21, 287-297 (1997).

190. Shakhova, O. \& Sommer, L. Neural crest-derived stem cells. (2008).

191. Liu, Y. et al. YAP modulates TGF-beta 1-induced simultaneous apoptosis and EMT through upregulation of the EGF receptor. Sci Rep-Uk 7 (2017).

192. Peinado, H., Olmeda, D. \& Cano, A. Snail, Zeb and bHLH factors in tumour progression: an alliance against the epithelial phenotype? Nat Rev Cancer 7, 415-428 (2007).

193. Zhao, D. et al. H3K4me3 Demethylase Kdm5a Is Required for NK Cell Activation by Associating with p50 to Suppress SOCS1. Cell Rep 15, 288299 (2016).

194. Kim, J.H., Lee, J.H., Lee, I.S., Lee, S.B. \& Cho, K.S. Histone Lysine Methylation and Neurodevelopmental Disorders. Int J Mol Sci 18 (2017).

195. Beshiri, M.L. et al. Coordinated repression of cell cycle genes by KDM5A and E2F4 during differentiation. Proc Natl Acad Sci U S A 109, 1849918504 (2012).

196. Lopez-Bigas, N. et al. Genome-wide analysis of the H3K4 histone demethylase RBP2 reveals a transcriptional program controlling differentiation. Mol Cell 31, 520-530 (2008).

197. Pasini, D. et al. Coordinated regulation of transcriptional repression by the RBP2 H3K4 demethylase and Polycomb-Repressive Complex 2. Genes Dev 22, 1345-1355 (2008).

198. Vallianatos, C.N. \& Iwase, S. Disrupted intricacy of histone H3K4 methylation in neurodevelopmental disorders. Epigenomics 7, 503-519 (2015).

199. Wynder, C., Stalker, L. \& Doughty, M.L. Role of H3K4 demethylases in complex neurodevelopmental diseases. Epigenomics 2, 407-418 (2010).

200. Lillico, R., Sobral, M.G., Stesco, N. \& Lakowski, T.M. HDAC inhibitors induce global changes in histone lysine and arginine methylation and alter expression of lysine demethylases. J Proteomics 133, 125-133 (2016).

201. Shakyawar, D.K., Dayma, K., Ramadhas, A., Varalakshmi, C. \& Radha, V. C3G shows regulated nucleocytoplasmic exchange and represses histone modifications associated with euchromatin. Mol Biol Cell 28, 984-995 (2017).

202. Chicas, A. et al. H3K4 demethylation by Jarid1a and Jarid1b contributes to retinoblastoma-mediated gene silencing during cellular senescence. Proc Natl Acad Sci U S A 109, 8971-8976 (2012).

203. Walport, L.J., Hopkinson, R.J. \& Schofield, C.J. Mechanisms of human histone and nucleic acid demethylases. Curr Opin Chem Biol 16, 525-534 (2012).

204. Serrano-Gomez, S.J., Maziveyi, M. \& Alahari, S.K. Regulation of epithelial-mesenchymal transition through epigenetic and posttranslational modifications. Mol Cancer 15, 18 (2016). 
205. Franco, D.L. et al. Snail1 suppresses TGF-beta-induced apoptosis and is sufficient to trigger EMT in hepatocytes. J Cell Sci 123, 3467-3477 (2010).

206. Han, B. et al. NUDCD1 promotes metastasis through inducing EMT and inhibiting apoptosis in colorectal cancer. Am J Cancer Res 8, 810-823 (2018).

207. Sauka-Spengler, T., Meulemans, D., Jones, M. \& Bronner-Fraser, M. Ancient evolutionary origin of the neural crest gene regulatory network. Dev Cell 13, 405-420 (2007).

208. Lamouille, S., Xu, J. \& Derynck, R. Molecular mechanisms of epithelialmesenchymal transition. Nat Rev Mol Cell Biol 15, 178-196 (2014).

209. Davies, P.F., Manduchi, E., Jimenez, J.M. \& Jiang, Y.Z. Biofluids, cell mechanics and epigenetics: Flow-induced epigenetic mechanisms of endothelial gene expression. J Biomech 50, 3-10 (2017).

210. Kushiro, K. \& Nunez, N.P. Ethanol inhibits B16-BL6 melanoma metastasis and cell phenotypes associated with metastasis. In Vivo 26, 47-58 (2012).

211. Smith, S.M., Garic, A., Berres, M.E. \& Flentke, G.R. Genomic factors that shape craniofacial outcome and neural crest vulnerability in FASD. Front Genet 5, 224 (2014).

212. Kong, D. et al. Histone deacetylase inhibitors induce epithelial-tomesenchymal transition in prostate cancer cells. PLoS One 7, e45045 (2012).

213. Natsume-Kitatani, Y. \& Mamitsuka, H. Classification of Promoters Based on the Combination of Core Promoter Elements Exhibits Different Histone Modification Patterns. PLoS One 11, e0151917 (2016).

214. Liang, G. et al. Distinct localization of histone $\mathrm{H} 3$ acetylation and $\mathrm{H} 3-\mathrm{K} 4$ methylation to the transcription start sites in the human genome. Proc Natl Acad Sci U S A 101, 7357-7362 (2004).

215. Hojfeldt, J.W., Agger, K. \& Helin, K. Histone lysine demethylases as targets for anticancer therapy. Nat Rev Drug Discov 12, 917-930 (2013).

216. Vogt, T. et al. Deficiency of a novel retinoblastoma binding protein 2homolog is a consistent feature of sporadic human melanoma skin cancer. Lab Invest 79, 1615-1627 (1999).

217. van Zutven, L.J. et al. Identification of NUP98 abnormalities in acute leukemia: JARID1A (12p13) as a new partner gene. Genes Chromosomes Cancer 45, 437-446 (2006).

218. Dimitrova, E., Turberfield, A.H. \& Klose, R.J. Histone demethylases in chromatin biology and beyond. EMBO Rep 16, 1620-1639 (2015).

219. Christensen, J. et al. RBP2 belongs to a family of demethylases, specific for tri-and dimethylated lysine 4 on histone 3. Cell 128, 1063-1076 (2007).

220. Seward, D.J. et al. Demethylation of trimethylated histone H3 Lys4 in vivo by JARID1 JmjC proteins. Nat Struct Mol Biol 14, 240-242 (2007).

221. Moore, E.S. et al. The subtle facial signs of prenatal exposure to alcohol: an anthropometric approach. J Pediatr 139, 215-219 (2001).

222. Moore, E.S. et al. Unique facial features distinguish fetal alcohol syndrome patients and controls in diverse ethnic populations. Alcohol Clin Exp Res 31, 1707-1713 (2007). 
223. Van Maele-Fabry, G., Gofflot, F., Clotman, F. \& Picard, J.J. Alterations of mouse embryonic branchial nerves and ganglia induced by ethanol. Neurotoxicol Teratol 17, 497-506 (1995).

224. Debelak, K.A. \& Smith, S.M. Avian genetic background modulates the neural crest apoptosis induced by ethanol exposure. Alcohol Clin Exp Res 24, 307-314 (2000).

225. Su, B., Debelak, K.A., Tessmer, L.L., Cartwright, M.M. \& Smith, S.M. Genetic influences on craniofacial outcome in an avian model of prenatal alcohol exposure. Alcohol Clin Exp Res 25, 60-69 (2001).

226. Delloye-Bourgeois, C., Rama, N., Brito, J., Le Douarin, N. \& Mehlen, P. Sonic Hedgehog promotes the survival of neural crest cells by limiting apoptosis induced by the dependence receptor CDON during branchial arch development. Biochem Biophys Res Commun 452, 655-660 (2014).

227. Jana, K., Jana, N., De, D.K. \& Guha, S.K. Ethanol induces mouse spermatogenic cell apoptosis in vivo through over-expression of Fas/FasL, p53, and caspase-3 along with cytochrome c translocation and glutathione depletion. Mol Reprod Dev 77, 820-833 (2010).

228. Hong, F. et al. Elevated interleukin- 6 during ethanol consumption acts as a potential endogenous protective cytokine against ethanol-induced apoptosis in the liver: involvement of induction of $\mathrm{Bcl}-2$ and $\mathrm{Bcl}-\mathrm{x}(\mathrm{L})$ proteins. Oncogene 21, 32-43 (2002).

229. Thibert, C. et al. Inhibition of neuroepithelial patched-induced apoptosis by sonic hedgehog. Science 301, 843-846 (2003).

230. Higashiyama, D. et al. Sequential developmental changes in holoprosencephalic mouse embryos exposed to ethanol during the gastrulation period. Birth Defects Res A Clin Mol Teratol 79, 513-523 (2007).

231. Parnell, S.E., Sulik, K.K., Dehart, D.B. \& Chen, S.Y. Reduction of ethanolinduced ocular abnormalities in mice through dietary administration of $\mathrm{N}$ acetylcysteine. Alcohol 44, 699-705 (2010).

232. Wentzel, P. \& Eriksson, U.J. Genetic influence on dysmorphogenesis in embryos from different rat strains exposed to ethanol in vivo and in vitro. Alcohol Clin Exp Res 32, 874-887 (2008).

233. Razin, A. \& Cedar, H. DNA methylation and gene expression. Microbiol Rev 55, 451-458 (1991).

234. Razin, A. \& Kantor, B. DNA methylation in epigenetic control of gene expression. Prog Mol Subcell Biol 38, 151-167 (2005).

235. Myzak, M.C., Karplus, P.A., Chung, F.L. \& Dashwood, R.H. A novel mechanism of chemoprotection by sulforaphane: inhibition of histone deacetylase. Cancer Res 64, 5767-5774 (2004).

236. Meeran, S.M., Patel, S.N. \& Tollefsbol, T.O. Sulforaphane causes epigenetic repression of hTERT expression in human breast cancer cell lines. PLoS One 5, e11457 (2010).

237. Hsu, A. et al. Promoter de-methylation of cyclin D2 by sulforaphane in prostate cancer cells. Clin Epigenetics 3, 3 (2011). 
238. Angelino, D. et al. Myrosinase-dependent and -independent formation and control of isothiocyanate products of glucosinolate hydrolysis. Front Plant Sci 6, 831 (2015).

239. Fahey, J.W., Zhang, Y. \& Talalay, P. Broccoli sprouts: an exceptionally rich source of inducers of enzymes that protect against chemical carcinogens. Proc Natl Acad Sci U S A 94, 10367-10372 (1997).

240. Subedi, L., Cho, K., Park, Y.U., Choi, H.J. \& Kim, S.Y. SulforaphaneEnriched Broccoli Sprouts Pretreated by Pulsed Electric Fields Reduces Neuroinflammation and Ameliorates Scopolamine-Induced Amnesia in Mouse Brain through Its Antioxidant Ability via Nrf2-HO-1 Activation. Oxid Med Cell Longev 2019, 3549274 (2019).

241. Clarren, S.K. Recognition of fetal alcohol syndrome. JAMA 245, 24362439 (1981).

242. Clarren, S.K. \& Smith, D.W. The fetal alcohol syndrome. N Engl J Med 298, 1063-1067 (1978).

243. Parnell, S.E. et al. Maternal oral intake mouse model for fetal alcohol spectrum disorders: ocular defects as a measure of effect. Alcohol Clin Exp Res 30, 1791-1798 (2006).

244. Parnell, S.E. et al. Concurrent dietary administration of D-SAL and ethanol diminishes ethanol's teratogenesis. Alcohol Clin Exp Res 31, 2059-2064 (2007).

245. Jackson, A.L. \& Linsley, P.S. Recognizing and avoiding siRNA off-target effects for target identification and therapeutic application. Nat Rev Drug Discov 9, 57-67 (2010).

246. Hannus, M. et al. siPools: highly complex but accurately defined siRNA pools eliminate off-target effects. Nucleic Acids Res 42, 8049-8061 (201 
LIST OF ABBREVIATIONS

FASD: Fetal Alcohol Spectrum Disorder

SFN: Sulforaphane

DNMT: DNA methyltransferase

HDAC: Histone deacetylase

KDM: Histone demethylase

H3ac: Histone $\mathrm{H} 3$ acetylation

H3K4me3: Trimethylation of histone H3 lysine 4

NCC: Neural crest cell

hNCC: human Neural creat cell

EMT: Epithelial-mesenchymal transition

KMT: Histone methyltransferase

HAT: Histone acetyltransferase

TET: DNA demethylase

BSE: Broccoli sprout extract

MSP: Methylated specific PCR

GD: Gestational Day

SAM: S-adenosylmethionine

VPA: Valproic acid

H3K27me3: Histone lysine 27 trimethylation 
hESC: human Embryonic stem cell

HDACi: Histone deacetylase inhibitor

H3K9ac: histone H3 lysine acetylation at position 9

TSA: Trichostain 


\title{
CURRICULUM VITAE
}

\author{
Yihong Li \\ 505 South Hancock Street, Room 533A \\ Louisville, KY 40202 \\ (502)-8077902 \\ yihong.li@louisville.edu
}

\section{Education}

2016- $\quad$ Ph. D candidate. Department of Pharmacology and Toxicology, present University of Louisville, Louisville, KY, US.

2012-2014 M. S. in Microbiology. Institute of Microbiology, Chinese Academy of Science, Beijing, China.

2011-2012 M. S. in Microbiology. Anhui University, Hefei, Anhui, China.

2007-2011 B. S. in Biology Engineering. Qiqihar University, Qiqihar, Heilongjiang, China.

\section{Work Experience}

2014-2016 Research associate, Wenzhou Institue of Biomaterials and Engineering, Wenzhou Institute, University of Chinese Academy of Sciences, Wenzhou. 


\section{Professional memberships}

2017-2019 Student member, Research Society on Alcoholism

2017 Student member, Society of Toxicology

\section{Honors and Awards}

2019 Student Merit Award, Research Society on Alcoholism

2018 Student Merit Award, Research Society on Alcoholism

2011-2012 Second Student Scholarship, Anhui University

\section{Abstracts and Presentations}

\section{Oral Presentations}

1. Research Seminar, University of Louisville, Seminar in Pharmacology and Toxicology, Louisville, KY. Jan. 2019

2. Research Seminar, University of Louisville, Seminar in Pharmacology and Toxicology, Louisville, KY. Dec. 2016

\section{Poster Presentations}

\section{Local/Regional}

1. Yihong Li, Fuqiang Yuan, Ting Wu, Lanhai Lu, Jie Liu, Shao-yu Chen. Epigenetically modulating the expression of anti-apoptotic genes by sulforaphane prevented ethanol-induced apoptosis in human neural crest cells. Research!Louisville, Louisville, KY. Oct. 2019. 
2. Yihong Li, Fuqiang Yuan, Ting Wu, Lanhai Lu, Jie Liu, Shao-yu Chen. Induction of Snail1 through down-regulation of lysine-specific histone demethylase KDM5A protects against ethanol-induced apoptosis in neural crest cells and zebrafish embryos. Research!Louisville, Louisville, KY. Oct. 2018

3. Yihong Li, Fuqiang Yuan, Ting Wu, Lanhai Lu, Jie Liu, Shao-yu Chen. Sulforaphane protects against ethanol-induced apoptosis in neural crest cells through restoring EMT by epigenetically modulating the expression of Snail1. Research!Louisville, Louisville, KY. Oct. 2017

\section{National}

1. Yihong Li, Fuqiang Yuan, Ting Wu, Lanhai Lu, Jie Liu, Shao-yu Chen. Sulforaphane protects against ethanol-induced apoptosis in human neural crest cells through epigenetically modulating the expression of antiapoptotic genes. Annual Scientific Meeting of the Research Society on Alcoholism. Minneapolis, Minnesota. June 2019

2. Yihong Li, Fuqiang Yuan, Ting Wu, Lanhai Lu, Jie Liu, Shao-yu Chen. Upregulation of lysine-specific demethylase KDM5A contributes to ethanolinduced apoptosis in neural crest cells and zebrafish embryos. Annual Scientific Meeting of the Research Society on Alcoholism., San Diego, California. June 2018

3. Yihong Li, Fuqiang Yuan, Ting Wu, Lanhai Lu, Jie Liu, Shao-yu Chen. Sulforaphane protects against ethanol-induced apoptosis in neural crest cells and zebrafish embryos through epigenetically modulating the 
expression of Snail1 and restoring EMT. Experimental Biology Meeting,, San Diego, California. April 2018

4. Yihong Li, Fuqiang Yuan, Ting Wu, Lanhai Lu, Jie Liu, Shao-yu Chen. Sulforaphane protects against ethanol-induced apoptosis in neural crest cells through epigenetically modulating the expression of Snail1 and restoring EMT. Society of Toxicology Meeting, San Antonio, Texas. March 2018

\section{Publications}

\section{As primary author}

1. Yihong Li, Fuqiang Yuan, Ting Wu, Lanhai Lu, Jie Liu, Shao-yu Chen. Sulforaphane protects against ethanol-induced apoptosis in neural crest cells through restoring epithelial-mesenchymal transition by epigenetically modulating the expression of Snail1. Biochim Biophys Acta Mol Basis Dis 1865, 2586-2594 (2019).

2. Yihong Li, Yuanhui Song, Waner Chen, Qien Xu, Zhe Liu. Hybrid polymeric nanoprobes for folate receptor-targeted photoacoustic imaging in vivo. Mat Chem Front 1, 916-921 (2017).

3. Yihong Li, Shanshan Li, Guomin Ai, Weishan Wang, Buchang Zhang, Keqian Yang. Optimized sample preparation for metabolome studies on Streptomyces coelicolor. Chinese Journal of Biotechnology 30, 554-568 (2014). 
4. Yihong Li, Fuqiang Yuan, Ting Wu, Lanhai Lu, Jie Liu, Shao-yu Chen. Upregulation of lysine-specific histone demethylase KDM5A contributes to ethanol-induced apoptosis in neural crest cells and zebrafish embryos. (In preparation)

5. Yihong Li, Fuqiang Yuan, Ting Wu, Lanhai Lu, Jie Liu, Shao-yu Chen. Maternal dietary administration of SFN-rich BSE represents a novel therapeutic strategy for the prevention of ethanol-induced teratogenesis. (In preparation)

6. Yihong Li, Fuqiang Yuan, Ting Wu, Lanhai Lu, Jie Liu, Shao-yu Chen. Sulforaphane protects against ethanol-induced apoptosis in human neural crest cells through epigenetically modulating the expression of antiapoptotic genes. (In preparation)

7. Yihong Li, Fuqiang Yuan, Ting Wu, Lanhai Lu, Jie Liu, Shao-yu Chen. Sulforaphane protects human neural crest cells from ethanol-induced apoptosis through targeting HDAC2 on the anti-apoptotic genes. (In preparation)

\section{As co-author}

1. Weishan Wang, Shanshan Li, Zilong Li, Jingyu Zhang, Keqiang Fan, Gaoyi Tan, Guomin Ai, Sin Man Lam, Guanghou Shui, Zhiheng Yang, Hongzhong Lu, Pinjiao Jin, Yihong Li, Xiangyin Chen, Xuekui Xia, Xueting Liu, H. Kathleen Dannelly, Chen Yang, Yi Yang, Siliang Zhang, Gli Alterovita, Wensheng Xiang, Lixin Zhang. Harnessing the intracellular triacylglycerols 
for titer improvement of polyketides in Streptomyces. Nat. Biotechnol. 38, $76-83(2020)$

2. Zhe Liu, Qien $\mathrm{Xu}$, Yihong Li, Waner Chen. Fluorescent C-dot nanocomposites as efficient photothermal agents and multi-modal imaging tracers. Mat Chem Front 1, 538-541 (2017).

3. Yuanhui Song, Yihong Li, Qien Xu, Zhe Liu. Mesoporous silica nanoparticles for stimuli-responsive controlled drug delivery: advances, challenges, and outlook. International journal of nanomedicine 12, 87-110 (2017).

4. Zhe Liu, Waner Chen, Yihong Li, Qien Xu. Integrin alpha(v)beta(3)Targeted C-Dot Nanocomposites as Multifunctional Agents for Cell Targeting and Photoacoustic Imaging of Superficial Malignant Tumors. Analytical Chemistry 88, 11955-11962 (2016).

5. Qien Xu, Yuanhui Song, Yihong Li, Zhe Liu. Nanocatalysis for Organic Chemistry. Curr Org Chem 20, 2013-2021 (2016).

6. Zhe Liu, Changcan Shi, Yihong Li, Yuanhui Song, Qien Xu. Fluorescent genipin cross-linked REDV-conjugated polymeric microbubbles for human vascular endothelial cell (HVEC) targeting. Rsc Adv6, 32710-32714 (2016).

7. Weishan Wang, Tongjian Yang, Yihong Li, Shanshan Li, Shouliang Yin, Kathryn Styles, Christophe Corre, Keqian Yang. Development of a Synthetic Oxytetracycline-Inducible Expression System for Streptomycetes Using de Novo Characterized Genetic Parts. ACS synthetic biology 5, 765773 (2016). 